\title{
Effects of school-based interventions on motivation towards physical activity in children and adolescents: A systematic review and meta-analysis
}

\author{
Anne Kelso ${ }^{\mathrm{a}, *}$, Stephanie Linder ${ }^{\mathrm{b}}$, Anne K. Reimers ${ }^{\mathrm{b}}$, Stefanie J. Klug ${ }^{\mathrm{c}}$, Marianna Alesi ${ }^{\mathrm{d}}$, \\ Lidia Scifo $^{\mathrm{d}}$, Carla Chicau Borrego ${ }^{\mathrm{e}}$, Diogo Monteiro ${ }^{\mathrm{f}, \mathrm{g}}$, Yolanda Demetriou ${ }^{\mathrm{a}}$ \\ ${ }^{\text {a }}$ Department of Sport and Health Sciences, Professorship of Educational Science in Sport and Health, Technical University of Munich, Germany \\ ${ }^{\mathrm{b}}$ Department of Sport Science and Sport, University Erlangen-Nuremberg, Germany \\ ${ }^{c}$ Department of Sport and Health Sciences, Chair of Epidemiology, Technical University of Munich, Germany \\ ${ }^{\mathrm{d}}$ Department of Psychology, Educational Science and Human Movement, University of Palermo, Italy \\ e Sport Sciences School of Rio Maior, Polytechnic Institute of Santarém Research Center in Life Quality (CIEQV), Santarém, Portugal \\ ${ }^{\mathrm{f}}$ Department of Human Kinetics - ESECS, Polytechnique of Leiria, Leiria, Portugal \\ ${ }^{g}$ Research Center in Sport, Health and Human Development (CIDESD), Vila Real, Portugal
}

\section{A R T I C L E I N F O}

\section{Keywords:}

Boys

Girls

Exercise

GRADE

Students

Youth

\begin{abstract}
A B S T R A C T
Introduction: Assuming that motivation is the key to initiate and sustain beneficial health behaviors, the aim of this systematic review was to analyze the effects of school-based physical activity interventions on a variety of motivational outcomes towards PA in school-aged children and adolescents.

Methods: A comprehensive literature search was carried out in six electronic databases to identify randomized controlled trials and quasi-experimental trials examining the effects of PA interventions implemented during the regular school day, e.g., during physical education lessons or lunch breaks. Primary outcomes of interest were students' motivation, basic psychological needs, goal orientation, enjoyment, and motivational teaching climate in physical education. Meta-analyses were conducted for these outcomes using Comprehensive Meta-analysis software. Secondarily, intervention effects on students' PA behaviors were examined and the findings summarized narratively. Methodological quality of studies was evaluated using the Cochrane Collaboration's tool for assessing risk of bias for randomized trials; certainty of evidence on outcome level was evaluated using the GRADE approach.

Results: In total, 57 studies carried out between 2001 and 2018 were included in this review. Sixteen individual meta-analyses were performed and revealed significant pooled effects for the outcomes enjoyment $(g=0.310)$, perceived autonomy $(g=0.152)$, identified regulation $(g=0.378)$, intrinsic motivation $(g=0.419)$, selfdetermination index $(g=0.672)$, task/mastery climate $(g=0.254)$, ego/performance climate $(g=-0.438)$, autonomy supportive climate $(g=0.262)$, task goal orientation $(g=1.370)$, ego goal orientation $(g=-0.188)$. The narrative data synthesis indicated an increase in students' PA behavior. The overall risk of bias was high across all studies and certainty of evidence of meta-analyzed outcomes ranged from very low to moderate. Moderate certainty of evidence was found for ego/performance climate and ego goal orientation. Conclusions: Meta-analyses suggest that school-based PA interventions may be effective in increasing a variety of motivational outcomes. However, the certainty of evidence was limited in the majority of outcomes. Further research is needed to identify effective intervention strategies that increase students' motivation towards PA.
\end{abstract}

Abbreviations: PA, physical activity; MVPA, moderate-to-vigorous physical activity; PE, physical education; SDT, Self-Determination Theory; AGT, Achievement

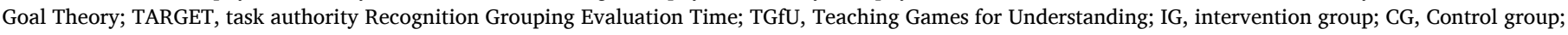
RCT, randomized controlled trial; CT, controlled trial; BCT, behaviour change technique; SDI, self-determination index; RAI, relative autonomy index.

* Corresponding author. Technical University of Munich, Department of Sport and Health Sciences, Georg-Brauchle-Ring 62, D-80992, Munich, Germany.

E-mail addresses: anne.kelso@tum.de (A. Kelso), stephanie.linder@fau.de (S. Linder), anne.reimers@fau.de (A.K. Reimers), stefanie.klug@tum.de (S.J. Klug),

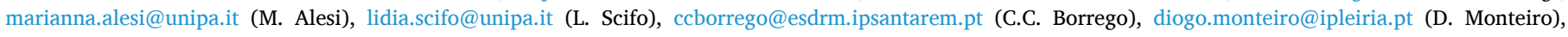
yolanda.demetriou@tum.de (Y. Demetriou). 


\section{Introduction}

Regular exercise and physical activity (PA) have been shown to benefit physical, mental, and social health as well as academic performance (Singh et al., 2012; Penedo and Dahn, 2005; Eime et al., 2013; Janssen and Leblanc, 2010). The World Health Organization has recommended $60 \mathrm{~min}$ of daily moderate-to-vigorous physical activity (MVPA) for children and adolescents aged five to 17 years (World Health Organization, 2010). However, in most European countries less than $50 \%$ of children and adolescents follow these guidelines according to self-reported PA data (Van Hecke et al., 2016). PA data collected in 13 to 15-year-old adolescents from 105 countries worldwide, revealed that $80.3 \%$ did not meet the recommended activity guidelines (Hallal et al., 2012).

The school setting is ideal for implementing PA interventions because entire groups of children and adolescents regardless of their socio-economic or migration status can be addressed (Demetriou et al., 2014) either in physical education (PE), regular academic lessons (Demetriou and Honer, 2012), recess (Ickes et al., 2013) or after school (Pate and O'Neill, 2009). Interventions promoting PA during PE, for example, have shown to increase the time spent being physically active as well as the overall participation in PE (van Sluijs et al., 2007). In addition to improved PA and motor performance, PA interventions implemented in the school setting were also found to benefit health outcomes and exercise knowledge in children and adolescents (Demetriou and Honer, 2012; van Sluijs et al., 2007; Demetriou et al., 2015). Examining the mediating factors of school-based PA behavior in youth, van Stralen et al., 2011 found evidence for a mediated effect of self-efficacy, intention, self-regulation, enjoyment, intrinsic motivation, and autonomy support on PA (van Stralen et al., 2011). Mediating variables explain the causal sequence between the intervention and the outcome, therefore the identification of relevant mediators can increase the effectiveness of future interventions (van Stralen et al., 2011). Intervention developers are advised to disclose the theoretical base of their intervention including the mediating variables and intervention strategies. It is then possible to draw conclusions as to which strategies are effective in changing mediators of a certain health behavior (van Stralen et al., 2011).

There are several theories that try to explain how a specific health behavior, such as PA, can be initiated and increased based upon a persons' motivation. The Self-Determination Theory (SDT) has been found suitable for developing health intervention programs and understanding the motivation of children and adolescents towards PA (Ng et al., 2012; Owen et al., 2014; Deci and Ryan, 2000). Within this theory, different types of motivation are characterized along the autonomy-control continuum, depending on the degree to which autonomous or controlled regulations are present (Deci and Ryan, 2000; Ryan and Deci, 2017). Amotivation is non-regulated and describes a state of passiveness with a lack of intentionality and motivation to act. Within extrinsic motivation, four types of regulation are described in which the degree of autonomous regulation increases from external regulation, introjected regulation, identified regulation to integrated regulation. Intrinsic motivation characterizes self-determined, autonomous behavior (Deci and Ryan, 2000; Ryan and Deci, 2017).

In order to develop more autonomous forms of motivation and engage in the desired behavior, SDT assumes that three basic psychological needs must be satisfied: autonomy, competence and relatedness (Deci and Ryan, 2000; Ryan et al., 2008). Autonomy describes the need to feel that the behavior is self-regulated and in accordance with the individual's values. Competence refers to a feeling of mastery and performing effectively. Relatedness refers to the need of feeling socially connected (Ryan and Deci, 2017). Autonomy and competence are crucial for the processes of internalization and integration of health beneficial behaviors such as PA (Deci and Ryan, 2000; Ryan et al., 2008). Relatedness further supports the process of internalization because individuals may be more responsive to behaviors that are promoted by persons which the individual feels connected to (Ryan et al., 2008). The satisfaction of these psychological needs has been associated with better health-related outcomes, such as increased PA (Ryan et al., 2008).

The Hierarchical Model of Intrinsic and Extrinsic Motivation by Vallerand (1997) considers intrinsic, extrinsic motivation and amotivation on a situational, contextual and global level. Motivation not only varies in its type but also in its level of generality. Specifically, situational motivation can be described as motivation during a specific task in $\mathrm{PE}$, whereas contextual motivation can be described as motivation towards PE in general. Global motivation describes the general motivation personality. The different types of motivation exist within the individual at the three different levels of generality (Vallerand, 1997). Almolda Tomas et al., 2014 assume that interventions successful in changing situational motivation could affect contextual motivation, i.e. $\mathrm{PE}$ in general and finally global motivation, leading to a healthy lifestyle.

A theory focusing on motivation within the classroom context is the Achievement Goal Theory (AGT), which links the classroom learning environment to classroom goals and student motivation (Ames and Archer, 1988). The goal orientations are grouped into performance and mastery goal orientation. Performance goal orientation is characterized by being successful without much effort, outperforming others, and being judged based upon ability. Mastery goal orientation is characterized by developing new skills, valuing the process of learning and attainment of mastery (Ames and Archer, 1988). These opposing goal orientations are evoked by different situational demands in the classroom and result in different motivational patterns (Ames and Archer, 1988). Goudas and Biddle (1994) examined the perception of achievement environment in PE, also referred to as motivational climate, and found that class learning orientation, perception of teacher support and perception of choice formed a mastery climate, which was the main predictor of intrinsic motivation in PE. A performance climate, characterized by class competition and worries about mistakes, was not a predictor of intrinsic motivation (Goudas and Biddle, 1994).

Based upon the introduced theories, several motivational teaching strategies have been developed to increase students' motivation towards PA within PE. These include amongst others: the Task, Authority, Recognition, Grouping, Evaluation, Time (TARGET) approach (Ames, 1992; Epstein et al., 1989), the Sport Education Model (Siedentop, 1994), and the Teaching Games for Understanding (TGfU) approach (Bunker and Thorpe, 1982).

Several studies have examined the effects of school-based PA interventions on selected motivational outcomes. For example, Dudley et al. (2011) evaluated the effects of PA interventions on enjoyment, which is linked to intrinsically motivated behavior (Ryan and Deci, 2017), and found inconclusive results due to poor methodological quality of the reviewed studies and their statistical power (Dudley et al., 2011). A meta-analysis by Burns et al. (2017) indicated significant effects of school-based intervention strategies on PA enjoyment with a true population effect of small-to-moderate magnitude. The examined studies focused on a student-centered approach, in which students were involved in decision-making and were offered choices regarding their activities. However, their results were limited due to the methodological heterogeneity of the evaluated studies (Burns et al., 2017). Braithwaite et al. (2011) showed that motivational climate interventions in PE had small overall treatment effects $(g=0.103)$ in groups exposed to mastery motivational climates. The largest treatment effects were found for behavioral outcomes (e.g. exercise frequency) $(g=0.39-0.49$ ), followed by affective (e.g. enjoyment) ( $g=-0.27$ to 0.59 ) and cognitive outcomes (e.g. competence, perception of motivational climate) $(g=-0.25$ to 0.32) (Braithwaite et al., 2011).

In summary, the literature suggests that theory-based PA interventions (van Stralen et al., 2011; Ng et al., 2012; Owen et al., 2014) implementing motivational strategies may have positive effects on PA behavior (Demetriou and Honer, 2012; Dudley et al., 2011; Burns et al., 
2017; Braithwaite et al., 2011). However, an evaluation of school-based PA intervention effects on different types of motivation, which are assumed to effect the quality and dynamics of behavior (Ryan and Deci, 2017; Quested et al., 2017), has not yet been performed. Therefore, the primary aim of this systematic review was to analyze the effects of school-based PA interventions on a variety of motivational outcomes towards PA in school-aged children and adolescents. Secondarily, the effects on students' PA behavior were examined.

\section{Methods}

\subsection{Inclusion criteria}

In this systematic review, any randomized (parallel group or cluster randomized) and quasi-experimental (controlled) trial examining the effects of a school-based PA intervention on students' motivation towards PA was included. The PA components were offered either in addition to regular PE, e.g. during morning, lunch or afternoon breaks, or as modified PE classes during the regular school day. Intervention components provided primarily after school or outside the school setting, e.g. community-based programs, were excluded. The studies had to aim their interventions at healthy school-aged children and adolescents between the ages of six to 19 years. Studies targeting populations with known health issues, e.g. students with overweight/obesity or mental disorders, were not considered. To determine intervention effects, the intervention group (IG) was compared to a control group (CG), which received the standard, traditional school curriculum or PE program. The outcomes of interest were measured before (at baseline) and after the intervention (post-test, follow-up). Studies not published in English language or in scientific journals, and grey literature were excluded from this review.

\subsection{Outcome measures}

Primarily, the eligible studies were required to provide a quantitative assessment of at least one aspect of students' motivation (e.g. intrinsic, extrinsic motivation, amotivation), basic psychological needs (autonomy, competence, relatedness), students' goal orientation, motivational climate in PE (task or performance climate), or enjoyment measured using questionnaires.

Secondary outcomes regarding PA behavior included the reporting of physical activity levels, step counts, exercise frequency and exercise duration in three domains: in-class, during the school day and in leisure time. Measurement tools were questionnaires, accelerometers, pedometers and direct observation.

\subsection{Literature search}

The literature search was carried out on October 18th, 2018 in the following six databases: Scopus (ELSEVIER), MEDLINE (Ovid), Eric (EBSCO), PsycInfo (EBSCO), Psyndex (EBSCO), SportDiscus (EBSCO). A comprehensive search strategy with a combination of keywords in the categories population, setting, sport, intervention method, and intervention objective was developed (Demetriou et al., 2019). An example of the search strategy for MEDLINE (Ovid) can be found in additional file 1. Contrary to the search strategy described in previously published systematic review protocol (Demetriou et al., 2019), we did not perform a search in the database Physical Education Index (ProQuest). This systematic review was registered in the PROSPERO international prospective register of systematic reviews (registration number: CRD42018110306).

\subsection{Study selection}

The database searches found 4453 publications, excluding duplicates. A minimum of two reviewers (YD, AK, and/or DR) independently screened and identified relevant articles. First, titles and abstracts were scanned based on the inclusion criteria. Then, full texts were examined in detail and screened for eligibility (Fig. 1). The reference lists of included articles were hand-searched to identify further relevant publications missed in the database searches. Any disagreements were resolved through discussion to meet a consensus among the reviewers. Ultimately, 57 studies were included for data extraction and risk of bias assessment. Records were managed with EndNote x8 (Clarivate Analytics, Philadelphia, PA, United States).

\subsection{Data extraction}

Two review authors (AK, DR) independently extracted the study details using a piloted data extraction form without being blinded to journals and authors. Extracted study details were: publication information, study design (as described in primary study), study population (i.e. participants' characteristics), intervention details for IG and CG (including study duration and frequency, theoretical background, teaching approach), measurement instruments, and results on primary and secondary outcomes. Additionally, the intervention strategies implemented at the student level (IG) were coded using the behavior change technique (BCT) taxonomy (BCT Taxonomy v1) described by Michie et al., 2013). A minimum of two reviewers (AK and MA, LS, CCB or DM) independently performed the coding of BCTs after an initial piloting. Reliability statistics were not calculated, inconsistencies were resolved by discussion. If information was missing or clarification of data was required, the authors of the primary studies were contacted via e-mail with a maximum of two contact attempts.

\subsection{Quality assessment}

Although this review was not limited to randomized controlled trials (RCT), the Cochrane Collaboration's tool for assessing risk of bias in randomized trials (Higgins and Green, 2011; Higgins et al., 2011) was used to assess methodological quality within studies. The review authors AK and DR performed the assessment of methodological quality independently. In cases of disagreements, a third review author (YD) was consulted, and uncertainties were discussed until a consensus was reached.

A critical assessment of selection bias (random sequence generation and allocation sequence concealment), performance bias (blinding of participants and personnel), detection bias (blinding of outcome assessment), attrition bias (incomplete outcome data), reporting bias (selective outcome reporting), and other potential sources of bias (baseline group differences, similar timing of measurements for all groups) was performed. Each entry was rated with either low, high or unclear risk of bias, the latter indicating a lack of information or uncertainty about potential bias. Studies claiming to perform a random group allocation to the IG and CG within the same school were rated with a high risk of bias. Studies describing a quasi-experimental study design, i.e. controlled trial (CT), were also rated with a high risk of bias regarding random sequence generation and allocation concealment; unless strict allocation concealment was described. In evaluating performance bias, a high risk of bias was determined when teachers conducted the intervention and/or students were aware of their group allocation. Studies with a follow-up of up to six months that reported less than $20 \%$ of attrition were rated with a low risk of bias for incomplete outcome data. For studies with a longer follow-up, the attrition rate should not have exceeded $30 \%$ to ensure a low risk of bias. Detection bias was rated high since questionnaires, i.e. self-report measures, were used to measure motivational outcomes and it was assumed that treatment groups were aware of a possible intervention. If the study did not report a comparison of baseline differences between groups, or if the reported differences in outcome variables were significant, a high risk of bias was presumed.

The certainty of evidence across studies was assessed on outcome 


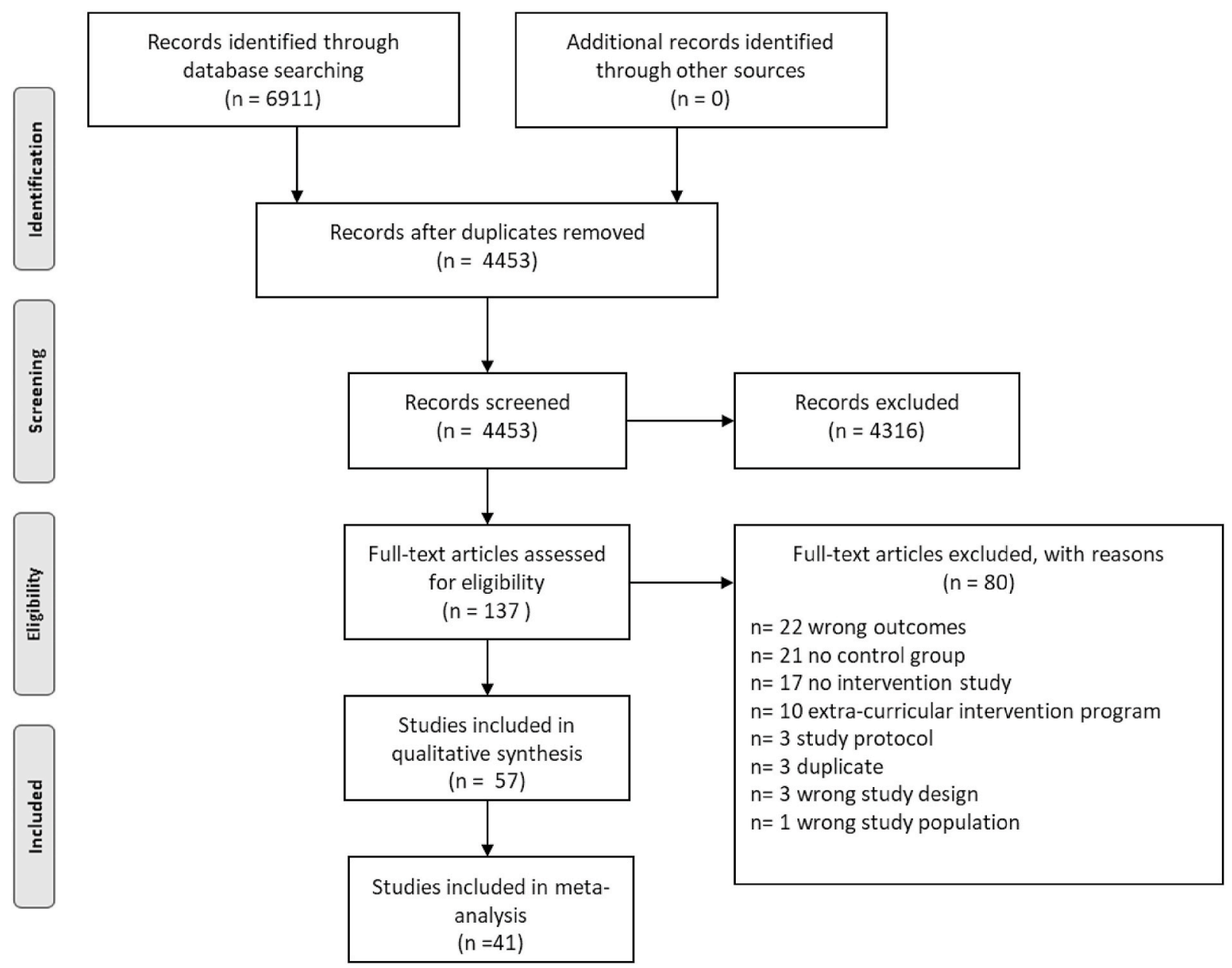

Fig. 1. Flowchart of the identification of included studies.

level using the GRADE approach (Guyatt et al., 2008; Schünemann et al., 2013). The certainty of evidence was graded per outcome based on the evaluation of following domains: study limitations (risk of bias), imprecision, heterogeneity, indirectness, and suspicion of publication bias. The GRADEpro GDT: GRADEpro Guideline Development Tool ( McMaster University,2020, developed by Evidence Prime, Inc.) available from gradepro.org was used to create the evidence table.

\subsection{Data synthesis-primary outcomes}

Meta-analytic procedures of primary outcomes were performed using Comprehensive Meta-analysis Software (version 3) (Biostat Inc. Englewood, New Jersey, USA) and included a calculation of pooled effect estimates, test of heterogeneity and analysis of publication bias.

Given that the outcomes of interest were continuous variables, the effect size Hedges' $g$ was used to represent the standard mean difference between the means of the IG and CG at post-test. Follow-up measurements were not considered. Hedges' $g$ has the advantage to be more accurate for small numbers of participants (Hedges and Olkin, 1985). Similar to Cohens' $d$, effect sizes of 0.8 were assumed to be large, effect sizes of 0.5 to be moderate, and effect sizes of 0.2 to be small (Cohen, 1988). Positive effect estimates indicated that IGs had increased, larger scores than CGs; negative effects indicated that IGs had reduced, smaller scores than CGs. For outcomes such as amotivation, ego goal orientation and ego/performance climate, negative effect estimates indicate better results of the IG compared to the CG. The main data entry format used for effect size calculation was mean, standard deviation and sample size for each group. If studies provided insufficient data to calculate Hedges' $g$, they were excluded from the analyses. A random effects model was chosen to do justice to heterogeneity across the studies (Hedges and Olkin, 1985; Hedges and Vevea, 1998). In cases of two subgroups for one outcome within the IG and CG, all data were extracted and combined to form one pooled effect size based on the Cochrane formulae for combining groups (Higgins and Green, 2011). When more than one IG was compared to a single CG within one study, they were considered as separate comparisons. Therefore, each comparison group was handled as a single study within the calculation process. To avoid double counting of controls in these cases, the sample size of the CG was divided by the number of the intervention groups (mean and standard deviation remained the same). A second IG participating in performance climate conditions was excluded from meta-analyses.

Heterogeneity was analyzed by calculating the $Q$-statistic and the $I^{2}$ statistic. $I^{2}$ is an indicator of heterogeneity on percentages. A value of $0 \%$ indicates no observed heterogeneity, and larger values show increasing heterogeneity, with $25 \%$ as low, $50 \%$ as moderate, and $75 \%$ as high heterogeneity (Higgins et al., 2003).

Publication bias was tested by a) visual inspection of the funnel plot on primary outcome measures (an asymmetric, as opposed to a symmetric inverted funnel shape, indicated potential publication bias); b) performing Egger's test of the intercept to quantify the bias captured by the funnel plot and tested whether it was significant $(p \leq 0.05)$.

The main analyses included effect size calculations for enjoyment, basic psychological needs, motivation,motivational climate, and goal orientation. The effect sizes were calculated for studies overall (combining CTs and RCTs) and for CTs and RCTs separately. In addition, subgroup-moderator analyses were conducted according to the mixed 
effect model. In this model, studies within subgroups are pooled with the random effects model, while tests for significant differences between subgroups are conducted with the fixed effects model. Two analyses concerning outliers were calculated by excluding a) studies with the highest and lowest effect size and b) studies with values of Hedges' $g$ not located within the $95 \%$ confidence interval of the random-effects model. Three further subgroup analyses were conducted: theory-based (yes/ no), age of participants (children/adolescents) and study design (RCT/ $\mathrm{CT}$ ). To do justice to the different study designs, the subgroup analyses were repeated for CTs only. Due to large differences in the numbers of comparisons in the meta-analyses, not all subgroup-moderator analyses were calculated for each variable.

\subsection{Data synthesis-secondary outcomes}

The results on PA outcomes were summarized narratively due to variances in the data collection tools (device-based and self-report measures), measurement time, and reported PA outcomes. The narrative summary was based on the reported effect estimates of included studies; $p$ values $\leq 0.05$ were considered significant.

\section{Results}

\subsection{General study characteristics}

The included studies in this systematic review were carried out between the years 2001 and 2018 (Barzouka, Sotiropoulos, \& Kioumourtzoglou, 2015; Blatsis et al., 2016; Braga, De Lima, Moyses, Moyses, \& Werneck, 2017; Brankovic \& Hadzikadunic, 2017; Bush, Laberge, \& Laforest, 2010; Chang, Chen, \& Tu, 2016; Chatzisarantis \& Hagger, 2009; Chiva-Bartoll, Salvador-García, \& Ruiz-Montero, 2018; Christodoulidis, Papaioannou, \& Digelidis, 2001; Cuevas, García- López, \& Serra- Olivares, 2016; Di Cagno, Crova, \& Pesce, 2006; Digelidis, Papaioannou, Laparidis, \& Christodoulidis, 2003; Dudley, Okely, Pearson, \& Peat, 2010; Elbe et al., 2017; S. Fairclough \& Stratton, 2005; S.J. Fairclough et al., 2016; Franco \& Coterón, 2017; Fu et al., 2013; Fu, Gao, Hannon, Burns, \& Brusseau, 2016; González-Cutre, Sierra, Beltrán-Carrillo, Peláez-Pérez, \& Cervelló, 2018; Gorely, Nevill, Morris, Stensel, \& Nevill, 2009; Harrington et al., 2018; Hobin, Subramaniam, \& Wuest, 2018; Hortigüela Alcalá \& Garijo, 2017; Jamner, Spruijt-Metz, Bassin, \& Cooper, 2004; Jones, Marshall, \& Peters, 2010; Kokkonen, Yli-Piipari, Kokkonen, \& Quay, 2018; Lonsdale et al., 2013, 2019; Méndez-Giménez, Fernández-Río, \& Méndez-Alonso, 2015; Meng \& Keng, 2016; Meng, Whipp, Dimmock, \& Jackson, 2013; Miller et al., 2016; Moreno, Gonzalez-Cutre, Martin-Albo, \& Cervello, 2010; Moreno-Murcia \& Sànchez-Latorre, 2016; Nation-Grainger, 2017; Palmer, Bycura, \& Warren, 2018; Pardo, Bengoechea, Clemente, \& Lanaspa, 2016; Patmanoglou, Mantis, \& Digelidis, 2008; D. Perlman, 2010; D.J. Perlman, 2011, 2015; Prusak, Treasure, Darst, \& Pangrazi, 2004; Salmon, Hume, \& Crawford, 2008; Sevil, Abos, Aibar, Julián, \& Garcia-Gonzalez, 2016; Shannon et al., 2018; J.J. Smith et al., 2018; L. Smith et al., 2015; Spittle \& Byrne, 2009; J. Todorovich R. \& Curtner-Smith, 2002; J.R. Todorovich \& Curtner-Smith, 2003; T. Wallhead L., Garn, \& Vidoni, 2014; T.L. Wallhead \& Ntoumanis, 2004; Wang, Baranowski, Lau, Buday, \& Gao, 2017; Weigand \& Burton, 2002; Yli-Piipari, Layne, Hinson, \& Irwin, 2018; Zach, Raviv, \& Meckel, 2016). Most studies were performed in the USA $(n=13)$, followed by Spain ( $n$ $=10)$, the UK $(n=8)$, Australia $(n=8)$, and Greece $(n=5)$. Regarding the study design, ten studies were identified as RCTs, 47 studies were CTs. Six studies included only female students, two studies only male students, and one further study did not describe the gender of included participants. The youngest participants were between six and seven years old (Di Cagno et al., 2006); the oldest students were aged 16.57 years (Dudley et al., 2010). We grouped the studies according to the students' age: participants with a mean age $( \pm \mathrm{SD})<12.5$ years were categorized as children and participants with a mean age $( \pm$ SD) $>$ than
12.5 years were categorized as adolescents. In total, 20 studies aimed their intervention at children, 37 studies targeted adolescents.

Regarding the study duration, most studies were of short duration ( $\leq$ three months $(\mathrm{n}=43)$ ), 11 studies of moderate duration (4-12 months) and three studies were longer than 12 months (Harrington et al., 2018; Pardo et al., 2016; Wallhead, Garn, \& Vidoni, 2014). Of the short-term studies, two examined motivational outcomes before the end of the intervention period, and the data from these intermediate measurements were used as post-values to examine possible intervention effects. This shortened the actual intervention period between baseline and final measurement to three and four weeks, respectively (Fairclough et al., 2016; Yli-Piipari et al., 2018). The sample sizes ranged from 10 students (Nation-Grainger, 2017) to 1752 (Harrington et al., 2018).

In total, 47 studies implemented modified PE classes and ten studies (Bush et al., 2010; Elbe et al., 2017; Fairclough et al., 2016; Gorely et al., 2009; Harrington et al., 2018; Pardo et al., 2016; Salmon et al., 2008; Shannon et al., 2018; Smith et al., 2018; Wang et al., 2017) offered additional PA programs throughout the school day. One of the studies providing modified PE classes additionally offered extracurricular PA components (González-Cutre et al., 2018). Diverse personnel including classroom and PE teachers, research staff, external health professionals and peers delivered the intervention components.

Eleven studies did not refer to an underlying theoretical framework. The two most commonly mentioned theories were SDT (Deci and Ryan, 2000) $(n=30)$ and AGT (Ames and Archer, 1988; Ames, 1992) $(n=11)$. Additionally, studies modified their teaching approaches to increase students' motivation towards PA. Most studies implemented self-designed teaching strategies, while others applied recognized approaches such as TARGET (Epstein et al., 1989) $(n=5)$, Sport Education Model (Siedentop, 1994) ( $\mathrm{n}=7$ ), or TGfU (Bunker and Thorpe, 1982) (n $=6)$.

In all studies, the IG was compared to a CG. The majority of studies reported that the CG participated in a regular, traditional PE condition or in a PE program without intervention components $(n=49)$. Eighteen studies provided some information on the contents of the control condition and largely described a traditional teacher-centered and teacherdirected approach focusing on skill development (Barzouka et al., 2015; Blatsis et al., 2016; Chang et al., 2016; Cuevas et al., 2016; Di Cagno et al., 2006; Fu et al., 2013; Hortigüela Alcalá and Garijo, 2017; Kokkonen, Yli-Piipari, Kokkonen, \& Quay, 2018; Méndez-Giménez et al., 2015; Meng et al., 2013; Perlman, 2010, 2011; Smith et al., 2015; Spittle and Byrne, 2009; Wallhead, Garn, \& Vidoni, 2014; Wallhead and Ntoumanis, 2004).

The included studies are described in detail in Table 1 and Table 2, separately for children and adolescents.

\subsection{Behavior change techniques}

It was possible to link at least one BCT to the described intervention components in all but four studies (Braga et al., 2017; Fu et al., 2016; Jones et al., 2010; Yli-Piipari et al., 2018). These four studies did not provide a detailed description of intervention components. The BCTs and corresponding clusters that were used in each study are shown in Tables 1 and 2. In total, 36 techniques were identified and allocated to twelve of the 16 main BCT clusters. The frequency of applied techniques within the main clusters varied across studies: 'Comparison of behavior' ( $\mathrm{n}=39$ ); 'Shaping knowledge' ( $\mathrm{n}=38)$; 'Repetition and substitution' ( $\mathrm{n}$ =37); 'Feedback and monitoring' ( $\mathrm{n}=28)$; 'Goals and planning' ( $\mathrm{n}=$ $20)$; 'Social support' $(n=12)$; 'Reward and threat' $(n=10)$; 'Natural consequences' $(n=6)$; 'Associations' $(n=3)$; 'Identity' $(n=3)$; 'Self-belief' $(n=1)$.

Studies grounded on the SDT most commonly applied BCTs within the clusters 'Comparison of behavior' $(\mathrm{n}=20)$ ); 'Shaping knowledge' ( $\mathrm{n}$ =19); 'Antecedents' ( $\mathrm{n}=19)$; 'Repetition and substitution' $(\mathrm{n}=18)$; 'Feedback and monitoring' $(\mathrm{n}=16)$.

Studies based on the AGT most frequently applied techniques within 
Table 1

Description of reviewed studies targeting children with examined outcomes at a minimum of two time points (pre to post).

\begin{tabular}{|c|c|c|c|c|c|c|c|}
\hline $\begin{array}{l}\text { Author; } \\
\text { CountryDesign }\end{array}$ & Participants & $\begin{array}{l}\text { Theoretical } \\
\text { background/ } \\
\text { Pedagogical } \\
\text { approach }\end{array}$ & Intervention description & Behavior change techniques & $\begin{array}{l}\text { Behavior change } \\
\text { technique cluster }\end{array}$ & Motivational outcomes & Physical activity outcomes \\
\hline $\begin{array}{l}\text { Blatsis et al. (2016); } \\
\text { GreeceCT }\end{array}$ & $\begin{array}{l}\mathrm{N}=215 \text { ( } 99 \text { boys) } \\
\text { 5th and 6th grade } \\
\text { students, age: } 11.61 \\
\text { (0.49) years. IG: } \\
\mathrm{n}=106 \text { (44 boys); } \\
\text { CG: } \mathrm{n}=109 \text { (55 } \\
\text { boys). }\end{array}$ & $\begin{array}{l}\text { SDT/Teaching } \\
\text { Games for } \\
\text { Understanding } \\
\text { (TGfU) }\end{array}$ & $\begin{array}{l}\text { IG: over } 12 \text { weeks, } \\
\text { students participated in } \\
\text { the International } \\
\text { Association of Athletics } \\
\text { Federation (IAAF) Kids' } \\
\text { Athletics program } \\
\text { promoting Track and } \\
\text { Field in schools and sport } \\
\text { clubs. } 24 \times \text { x } 45 \text {-minute } \\
\text { sessions were offered } \\
\text { twice per week. } \\
\text { Instructions were based } \\
\text { on a games-centered- } \\
\text { approach. CG: students } \\
\text { participated in track and } \\
\text { field lessons following a } \\
\text { traditional skill teaching } \\
\text { approach. }\end{array}$ & $\begin{array}{l}\text { 4.1. Instruction on how to } \\
\text { perform the behavior6.1. } \\
\text { Demonstration of the } \\
\text { behavior } 6.2 \text {. Social } \\
\text { comparison } \\
\text { 8.1. Behavioral practice/ } \\
\text { rehearsal12.2. Restructuring } \\
\text { the social environment }\end{array}$ & $\begin{array}{l}\text { 4. Shaping knowledge6. } \\
\text { Comparison of } \\
\text { behavior8. Repetition } \\
\text { and substitution12. } \\
\text { Antecedents }\end{array}$ & $\begin{array}{l}\text { intrinsic motivation } * * \text { identified regulation } * * \text { introjected } \\
\text { regulationexternal regulation amotivation SDI** } \\
\text { enjoyment/interest }\end{array}$ & $\mathrm{x}$ \\
\hline $\begin{array}{l}\text { Braga et al. (2017); } \\
\text { BrazilCT }\end{array}$ & $\begin{array}{l}\mathrm{N}=117 \text { (57 boys) } \\
\text { 3rd and 5th grade } \\
\text { students. IG: } \mathrm{n}=60 \\
\text { ( } 26 \text { boys), age: } 10 \\
\text { (0.9) years; CG: } \\
\mathrm{n}=57 \text { ( } 31 \text { boys), } \\
\text { age }=10(2.3) \text { years. }\end{array}$ & $\begin{array}{l}\text { Flow: Psychology } \\
\text { of optimal } \\
\text { experience }\end{array}$ & $\begin{array}{l}\text { IG: over } 12 \text { weeks, } \\
\text { students participated in } \\
\text { weekly PE classes using } \\
\text { Exergames (visual } \\
\text { gaming). CG: students } \\
\text { participated in traditional } \\
\text { PE classes. }\end{array}$ & No information & No information & motivation at beginning and end of each PE class & $\begin{array}{l}\text { in-class MPA (observation) } \\
(\%)^{* *}\end{array}$ \\
\hline $\begin{array}{l}\text { Di Cagno et al. } \\
\text { (2006); ItalyCT }\end{array}$ & $\begin{array}{l}\mathrm{N}=178 \text { ( } 94 \text { boys) six } \\
\text { to seven year old } \\
\text { primary school } \\
\text { students. IG: } \mathrm{n}=90 \text {, } \\
\text { CG: } \mathrm{n}=88 \text {. }\end{array}$ & $\begin{array}{l}\text { child-centered } \\
\text { teaching involving } \\
\text { indirect creative } \\
\text { methods and/or } \\
\text { reciprocal and } \\
\text { guided discovery } \\
\text { styles }\end{array}$ & $\begin{array}{l}\text { IG: over three months, } \\
\text { students participated in } \\
\text { PE lessons twice a week } \\
\text { ( } 2.5 \text { hours each) following } \\
\text { a student-centered } \\
\text { teaching style based on } \\
\text { creative discovery and } \\
\text { variability of coordination } \\
\text { demands coupled with a } \\
\text { problem-solving approach } \\
\text { to motor learning. CG: } \\
\text { students participated in } \\
\text { regular PE classes with a } \\
\text { teacher-focused style of } \\
\text { teaching motor skills via } \\
\text { demonstration and } \\
\text { command. }\end{array}$ & $\begin{array}{l}\text { 1.2. Problem solving4.1. } \\
\text { Instruction on how to } \\
\text { perform the behavior } 6.1 \text {. } \\
\text { Demonstration of the } \\
\text { behavior8.1. Behavioral } \\
\text { practice/rehearsal12.5. } \\
\text { Adding objects to the } \\
\text { environment }\end{array}$ & $\begin{array}{l}\text { 1. Goals and planning } 4 . \\
\text { Shaping knowledge6. } \\
\text { Comparison of } \\
\text { behavior8. Repetition } \\
\text { and substitution12. } \\
\text { Antecedents }\end{array}$ & $\begin{array}{l}\text { enjoyment rhythmic gymnastics*** enjoyment track and } \\
\text { field task }{ }^{* *}\end{array}$ & $\mathrm{x}$ \\
\hline $\begin{array}{l}\text { Digelidis et al. } \\
\text { (2003); GreeceCT }\end{array}$ & $\begin{array}{l}\mathrm{N}=783 \text { 7th grade } \\
\text { students. IG: } \mathrm{n}=262 \\
\text { (130 boys), age: } \\
11.88 \text { (0.6); CG: } \\
\mathrm{n}=521 \text { ( } 245 \text { boys), } \\
\text { age: } 12.14(0.77) .\end{array}$ & $\begin{array}{l}\text { Goal Perspectives } \\
\text { Theory/ TARGET }\end{array}$ & $\begin{array}{l}\text { IG: over one year, } \\
\text { students took part in PE } \\
\text { lessons } 3 \text { times a week for } \\
45 \text { minutes based on the } \\
\text { TARGET model, cognitive } \\
\text { strategies (e.g. mental } \\
\text { rehearsal), and a personal } \\
\text { goal-setting program. } \\
\text { Teachers participated in }\end{array}$ & $\begin{array}{l}\text { 1.3. Goal setting (outcome) } \\
\text { 2.2. Feedback on behavior } \\
\text { 4.1. Instruction on how to } \\
\text { perform the behavior 5.1. } \\
\text { Information about health } \\
\text { consequences5.6. } \\
\text { Information about } \\
\text { emotional consequences6.1. } \\
\text { Demonstration of the }\end{array}$ & $\begin{array}{l}\text { 1. Goals and planning2. } \\
\text { Feedback and } \\
\text { monitoring4. Shaping } \\
\text { knowledge5. Natural } \\
\text { consequences } 6 . \\
\text { Comparison of } \\
\text { behavior8. Repetition } \\
\text { and substitution12. }\end{array}$ & $\begin{array}{l}\text { task-involving climate*ego-involving climate**ask } \\
\text { orientation ego orientation* } \\
\text { athletic ability/competenceenjoyment }\end{array}$ & $\begin{array}{l}\text { self-reported exercise } \\
\text { frequency }\end{array}$ \\
\hline
\end{tabular}

v 


\begin{tabular}{|c|c|c|c|c|c|c|c|}
\hline $\begin{array}{l}\text { Author; } \\
\text { CountryDesign }\end{array}$ & Participants & $\begin{array}{l}\text { Theoretical } \\
\text { background/ } \\
\text { Pedagogical } \\
\text { approach }\end{array}$ & Intervention description & Behavior change techniques & $\begin{array}{l}\text { Behavior change } \\
\text { technique cluster }\end{array}$ & Motivational outcomes & Physical activity outcomes \\
\hline & & & $\begin{array}{l}\text { seminars and meetings in } \\
\text { order to successfully } \\
\text { implement the task } \\
\text { involving teaching } \\
\text { climate. CG: students } \\
\text { participated in PE. }\end{array}$ & $\begin{array}{l}\text { behavior8.1. Behavioral } \\
\text { practice/rehearsal12.2. } \\
\text { Restructuring the social } \\
\text { environment15.2. Mental } \\
\text { rehearsal of successful } \\
\text { performance15.4. Self-talk }\end{array}$ & $\begin{array}{l}\text { Antecedents15. Self- } \\
\text { belief }\end{array}$ & & \\
\hline $\begin{array}{l}\text { Elbe et al. (2017); } \\
\text { DenmarkRCT }\end{array}$ & $\begin{array}{l}\mathrm{N}=300 \text { 3rd grade } \\
\text { students ( } 47.3 \% \\
\text { boys), age: } 9.30 \\
\text { (0.35). } \mathrm{IG}_{1}: \mathrm{n}=111 \\
\text { ( } 51 \text { boys), } \mathrm{IG}_{2}: \\
\mathrm{n}=104 \text { ( } 49 \text { boys), } \\
\text { CG: } \mathrm{n}=85 \text { ( } 42 \text { boys). }\end{array}$ & no information & $\begin{array}{l}\mathrm{IG}_{1} \text { : over } 10 \text { months, } \\
\text { students participated in a } \\
\text { team sport intervention of } \\
\text { small-sided football and } \\
\text { other ball games. In half } \\
\text { of the schools team sports } \\
\text { were conducted in } 3 \\
\text { sessions per week, } 40 \\
\text { minutes each. The other } \\
\text { half of schools received } 5 \\
\text { sessions per week, } 12 \\
\text { minutes each. } \mathrm{IG}_{2} \text { : } \\
\text { students participated in } \\
\text { an individual sport } \\
\text { intervention (circuit } \\
\text { strength training, interval } \\
\text { running). Strength } \\
\text { training sessions were } \\
\text { held } 3 \text { times a week for } 40 \\
\text { minutes. Interval running } \\
\text { took place } 5 \text { times a week, } \\
12 \text { minutes per session. } \\
\text { Training sessions were } \\
\text { held by instructors } \\
\text { (university staff) in } \\
\text { addition to regular PE (90 } \\
\text { minutes per week). CG: } \\
\text { students continued with } \\
\text { regular PE. }\end{array}$ & $\begin{array}{l}\text { 4.1. Instruction on how to } \\
\text { perform the behavior6.1. } \\
\text { Demonstration of the } \\
\text { behavior8.1. Behavioral } \\
\text { practice/rehearsal12.2. } \\
\text { Restructuring the social } \\
\text { environment }\end{array}$ & $\begin{array}{l}\text { 4. Shaping knowledge6. } \\
\text { Comparison of } \\
\text { behavior8. Repetition } \\
\text { and substitution12. } \\
\text { Antecedents }\end{array}$ & enjoymentsocial cohesion (relatedness) & $\mathrm{x}$ \\
\hline \multirow[t]{2}{*}{$\begin{array}{l}\text { Fairclough \& } \\
\text { Stratton (2005); } \\
\text { EnglandCT }\end{array}$} & $\begin{array}{l}\mathrm{N}=26 \text { 7th grade } \\
\text { female students, } \\
\text { age: } 11-12 \text { years. IG: } \\
\mathrm{n}=12 \text {; CG: } \mathrm{n}=14 \text {. }\end{array}$ & no information & $\begin{array}{l}\text { IG: students participated } \\
\text { in } 6 \text { lesson units of } \\
\text { gymnastics once a week, } \\
120 \text { minutes each. The } \\
\text { teacher was instructed to } \\
\text { enhance PA following the } \\
\text { unit of work and planning } \\
\text { lessons according to the } \\
\text { objectives: organization } \\
\text { of groups and use of } \\
\text { space, equipment and } \\
\text { resources; teaching } \\
\text { approaches; lesson pace; } \\
\text { teacher positioning; } \\
\text { active learning; having } \\
\text { fun. CG: students }\end{array}$ & $\begin{array}{l}\text { 2.2. Feedback on } \\
\text { behavior4.1. Instruction on } \\
\text { how to perform the } \\
\text { behavior6.1. Demonstration } \\
\text { of the behavior8.1. } \\
\text { Behavioral practice/ } \\
\text { rehearsal12.2. Restructuring } \\
\text { the social environment }\end{array}$ & $\begin{array}{l}\text { 2. Feedback and } \\
\text { monitoring4. Shaping } \\
\text { knowledge6. } \\
\text { Comparison of } \\
\text { behavior8. Repetition } \\
\text { and substitution12. } \\
\text { Antecedents }\end{array}$ & intrinsic motivation competence & $\begin{array}{l}\text { in-class MVPA (\%) } \\
\text { (observation)* }\end{array}$ \\
\hline & & & & & & & (continued on next page) \\
\hline
\end{tabular}

meetings in behavior8.1. Behaviora environment15.2. Menta participated in PE $\quad$ performance15.4. Self-talk : over 10 months, 4.1. Instruction on how to mall-sided football and behaviors. Behavio students participated in individual sport rength training, interv running). Strength

held 3 times a week for 40 2 minutes per session. raining sessions were iniversity staff) in addition to regular PE (90 minutes per week). CG: in 6 lesson units of

2.2. Feedback on behavior4.1. Instru knowledge6. lessons according to the f groups and use of teacher positioning; ctive learning; having 
Table 1 (continued)

\begin{tabular}{|c|c|c|c|c|c|c|c|}
\hline $\begin{array}{l}\text { Author; } \\
\text { CountryDesign }\end{array}$ & Participants & $\begin{array}{l}\text { Theoretical } \\
\text { background/ } \\
\text { Pedagogical } \\
\text { approach }\end{array}$ & Intervention description & Behavior change techniques & $\begin{array}{l}\text { Behavior change } \\
\text { technique cluster }\end{array}$ & Motivational outcomes & Physical activity outcomes \\
\hline & & & $\begin{array}{l}\text { participated in regular PE } \\
\text { classes. }\end{array}$ & & & & \\
\hline $\begin{array}{l}\text { Fairclough et al. } \\
\text { (2016); EnglandCT }\end{array}$ & $\begin{array}{l}\mathrm{N}=139 \text { year } \text { six } \\
\text { students: } \mathrm{IG}: \mathrm{n}=73 \text {, } \\
\text { age: } 10.7 ; \mathrm{CG}: \mathrm{n}=66 \text {, } \\
\text { age: } 10.7 \text { years. }\end{array}$ & $\begin{array}{l}\text { Youth Physical } \\
\text { Activity Promotion } \\
\text { Model }\end{array}$ & $\begin{array}{l}\text { IG: over } 6 \text { weeks, students } \\
\text { received a PA } \\
\text { intervention called "Born } \\
\text { to Move (BTM) twice a } \\
\text { week for } 30 \text { minutes. BTM } \\
\text { is an age-adapted } \\
\text { portfolio of class-based PA } \\
\text { and fitness programs set } \\
\text { to music with an emphasis } \\
\text { on enjoyable and } \\
\text { inclusive activity. BTM } \\
\text { lessons were offered in } \\
\text { addition to regular PE and } \\
\text { led by a trained BTM } \\
\text { instructor.CG: students } \\
\text { received regular PE } \\
\text { classes twice a week for } \\
\text { 30-45 minutes. }\end{array}$ & $\begin{array}{l}\text { 4.1. Instruction on how to } \\
\text { perform the behavior6.1. } \\
\text { Demonstration of the } \\
\text { behavior8.1. Behavioral } \\
\text { practice/ rehearsal10.2. } \\
\text { Material reward (behavior) } \\
\text { 12.1. Restructuring the } \\
\text { physical environment }\end{array}$ & $\begin{array}{l}\text { 4. Shaping knowledge6. } \\
\text { Comparison of } \\
\text { behavior8. Repetition } \\
\text { and substitution } 10 . \\
\text { Reward and threat12. } \\
\text { Antecedents }\end{array}$ & At mid-term ( 3 weeks)enjoyment*competence & 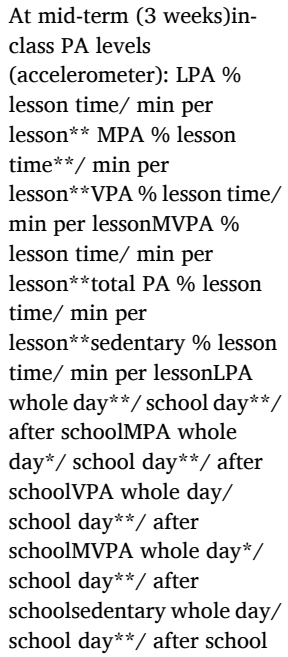 \\
\hline $\begin{array}{l}\text { Gorely et al. (2009); } \\
\text { EnglandCT }\end{array}$ & $\begin{array}{l}\mathrm{N}=589 \text { primary } \\
\text { school students. IG: } \\
\mathrm{n}=310(150 \text { boys), } \\
\text { age: } 8.8 \text { years;CG: } \\
\mathrm{n}=279(137 \text { boys), } \\
\text { age: } 8.9 \text { years }\end{array}$ & $\begin{array}{l}\text { Social Cognitive } \\
\text { Theory }\end{array}$ & $\begin{array}{l}\text { IG: over } 10 \text { months, } \\
\text { students participated in } \\
\text { the "GreatFun2Run" } \\
\text { program containing PE } \\
\text { lessons, PA events and } \\
\text { classroom activities. PE } \\
\text { lessons were held twice a } \\
\text { week. The program was } \\
\text { multifaceted and } \\
\text { comprised a CD-ROM } \\
\text { learning and teaching } \\
\text { resource for teachers; an } \\
\text { interactive website for } \\
\text { students, teachers, and } \\
\text { parents; two highlight PA } \\
\text { events (1 mile school } \\
\text { runs/walks); a local } \\
\text { media campaign; and a } \\
\text { summer activity wall } \\
\text { planner and record. CG: } \\
\text { students continued with } \\
\text { usual PE and health } \\
\text { curriculum, two hours per } \\
\text { week. }\end{array}$ & $\begin{array}{l}\text { 1.1. Goal setting (behavior) } \\
\text { 2.3. Self-monitoring of } \\
\text { behavior3.2. Social support } \\
\text { (practical) 5.1. Information } \\
\text { about health } \\
\text { consequences8.1. } \\
\text { Behavioral practice/ } \\
\text { rehearsal8.2. Behavior } \\
\text { substitution8.3. Habit } \\
\text { formation8.4. Habit reversal }\end{array}$ & $\begin{array}{l}\text { 1. Goals and planning } 2 . \\
\text { Feedback and } \\
\text { monitoring3. Social } \\
\text { support5. Natural } \\
\text { consequences8. } \\
\text { Repetition and } \\
\text { substitution }\end{array}$ & $\begin{array}{l}\text { competenceenjoyment of PA intrinsic motivationextrinsic } \\
\text { motivation* }\end{array}$ & $\begin{array}{l}\text { steps/day (pedometer) } \\
{ }^{* *} \text { MVPA min/day } \\
\text { (accelerometer) }^{* * *}\end{array}$ \\
\hline
\end{tabular}

\section{participated in regular PE}

behavior8. Repet

students: IG: $\mathrm{n}=73$, Action $10.7 ; \mathrm{CG}: \mathrm{n}=66$, Mode

week for 30 min

Reward and threat 12 .

Material reward (behavio

on enjoyable an

clusive activity. BT

addition to regular PE and

led by a trained BT

received regular PE

classes twice a week fo

30-45 minutes.

: over 10 months,

udents participated 


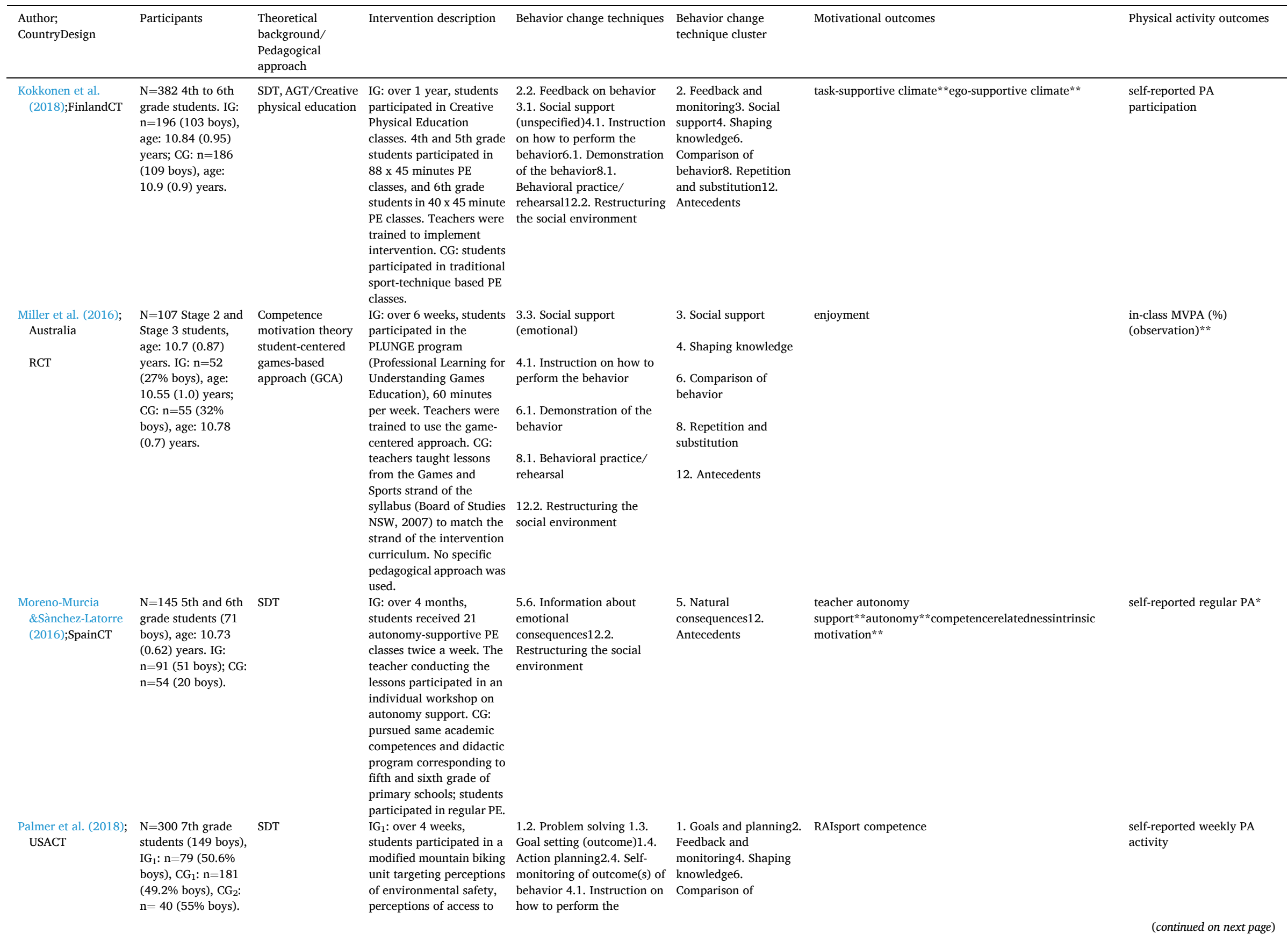


Table 1 (continued)

\begin{tabular}{|c|c|c|c|c|c|c|c|}
\hline $\begin{array}{l}\text { Author; } \\
\text { CountryDesign }\end{array}$ & Participants & $\begin{array}{l}\text { Theoretical } \\
\text { background/ } \\
\text { Pedagogical } \\
\text { approach }\end{array}$ & Intervention description & Behavior change techniques & $\begin{array}{l}\text { Behavior change } \\
\text { technique cluster }\end{array}$ & Motivational outcomes & Physical activity outcomes \\
\hline & & & $\begin{array}{l}\text { equipment, efficacy for } \\
\text { removing barriers, } \\
\text { perceived sport } \\
\text { competence, and sport } \\
\text { ability beliefs. Teachers } \\
\text { co-designed the learning } \\
\text { activites. } \mathrm{CG}_{1} \text { : students } \\
\text { participated in regular PE } \\
\text { classes. } \mathrm{CG}_{2} \text { s students did } \\
\text { not participate in any PE } \\
\text { classes. }\end{array}$ & $\begin{array}{l}\text { behavior6.1. Demonstration } \\
\text { of the behavior8.1. } \\
\text { Behavioral practice/ } \\
\text { rehearsal }\end{array}$ & $\begin{array}{l}\text { behavior8. Repetition } \\
\text { and substitution }\end{array}$ & & \\
\hline $\begin{array}{l}\text { Pardo et al. (2016); } \\
\text { SpainCT }\end{array}$ & $\begin{array}{l}\mathrm{N}=553 \text { secondary } \\
\text { school students ( } 292 \\
\text { boys). Cohort } 1: \\
\text { age }=12.03(0.16) \\
\text { years. IG: } \mathrm{n}=104 \text {, } \\
\text { CG: } \mathrm{n}=100 . \\
\text { Cohort2: age }= \\
12.07(0.26) \text { years. } \\
\text { IG: } \mathrm{n}=96, \mathrm{CG}: \\
\mathrm{n}=80 . \text { Cohort3: } \\
\text { age= 12.05 (0.21) } \\
\text { years, IG: } \mathrm{n}=10, \mathrm{CG}: \\
\mathrm{n}=71 .\end{array}$ & $\begin{array}{l}\text { SDT, Social } \\
\text { ecological model } \\
\text { of health-related } \\
\text { behavior }\end{array}$ & $\begin{array}{l}\text { IG: over three school } \\
\text { years, students took part } \\
\text { in a PA intervention } \\
\text { operationalized through } \\
\text { curricular and non- } \\
\text { curricular channels. } \\
\text { Curricular channel: } \\
\text { classroom teachers } \\
\text { received training in PA } \\
\text { promotion, which } \\
\text { involved students in } \\
\text { designing of PA-related } \\
\text { activities and initiatives } \\
\text { (e.g., at recess, during or } \\
\text { after school hours), } \\
\text { specific actions targeting } \\
\text { teacher behavior in PE } \\
\text { and a student project. } \\
\text { Non-curricular channel: } \\
\text { information } \\
\text { dissemination of activities } \\
\text { through which school } \\
\text { members, families, and } \\
\text { the community were } \\
\text { updated on the } \\
\text { intervention; } \\
\text { participation in } \\
\text { institutional programs } \\
\text { and special PA events in } \\
\text { the community to connect } \\
\text { schools with community } \\
\text { organizations and } \\
\text { programs. CG: schools did } \\
\text { not receive intervention. }\end{array}$ & $\begin{array}{l}\text { 7.1. Prompts/cues } 12.2 \text {. } \\
\text { Restructuring the social } \\
\text { environment }\end{array}$ & $\begin{array}{l}\text { 7. Associations12. } \\
\text { Antecedents }\end{array}$ & $\begin{array}{l}\text { enjoyment**intrinsic motivation**extrinsic } \\
\text { motivation*amotivation* } \\
\text { autonomy**}{ }^{* *} \text { competence }^{* * *} \text { relatedness }\end{array}$ & $\begin{array}{l}\text { MVPA (min/day) } \\
\text { (accelerometer)** }\end{array}$ \\
\hline
\end{tabular}




\begin{tabular}{|c|c|c|c|c|c|c|c|}
\hline $\begin{array}{l}\text { Author; } \\
\text { CountryDesign }\end{array}$ & Participants & $\begin{array}{l}\text { Theoretical } \\
\text { background/ } \\
\text { Pedagogical } \\
\text { approach }\end{array}$ & Intervention description & Behavior change techniques & $\begin{array}{l}\text { Behavior change } \\
\text { technique cluster }\end{array}$ & Motivational outcomes & Physical activity outcomes \\
\hline $\begin{array}{l}\text { Patmanoglou et al. } \\
\text { (2008);GreeceCT }\end{array}$ & $\begin{array}{l}\mathrm{N}=3075 \text { th and 6th } \\
\text { grade students }(160 \\
\text { boys), age: } 11(0.5) \\
\text { years. }\end{array}$ & $\begin{array}{l}\text { Spectrum of } \\
\text { teaching styles }\end{array}$ & $\begin{array}{l}\mathrm{IG}_{1} \text { : over three months, } \\
\text { students participated in } \\
10 \text { weekly lessons of } \\
\text { tennis in accordance with } \\
\text { the command teaching } \\
\text { style. IG } \mathrm{I}_{2}: \text { students } \\
\text { attended } 10 \text { tennis lessons } \\
\text { following the self-check } \\
\text { teaching style. CG: } \\
\text { students did not receive } \\
\text { intervention. }\end{array}$ & $\begin{array}{l}\text { 2.3. Self-monitoring of } \\
\text { behavior4.1. Instruction on } \\
\text { how to perform the } \\
\text { behavior6.1. Demonstration } \\
\text { of the behavior8.1. } \\
\text { Behavioral practice/ } \\
\text { rehearsal }\end{array}$ & $\begin{array}{l}\text { 2. Feedback and } \\
\text { monitoring } 4 \text {. Shaping } \\
\text { knowledge6. } \\
\text { Comparison of } \\
\text { behavior8. Repetition } \\
\text { and substitution }\end{array}$ & enjoyment* athletic ability/competence*** & $\mathrm{x}$ \\
\hline $\begin{array}{l}\text { Salmon et al. (2008); } \\
\text { AustraliaRCT }\end{array}$ & $\begin{array}{l}\mathrm{N}=295 \text { 5th grade } \\
\text { students ( } 49 \% \\
\text { boys), age: } 10.8 \\
\text { years. } \mathrm{IG}_{1}: \mathrm{n}=74 ; \\
\mathrm{IG}_{2}: \mathrm{n}=66 ; \mathrm{IG}_{3}: \\
\mathrm{n}=93 ; \mathrm{CG}: \mathrm{n}=62 .\end{array}$ & $\begin{array}{l}\text { Social cognitive } \\
\text { theory, Behavioral } \\
\text { choice theory }\end{array}$ & $\begin{array}{l}\text { Over } 1 \text { school year, PA } \\
\text { intervention components } \\
\text { were delivered in addition } \\
\text { to usual PE and sport } \\
\text { classes by one PE teacher. } \\
\text { IG }_{1} \text { : students participated } \\
\text { in } 19 \text { behavioral } \\
\text { modification lessons } \\
\text { delivered in the } \\
\text { classroom, } 40-50 \text { minutes } \\
\text { each. IG } \text { I students took }_{\text {part in } 19 \text { lessons focusing }} \\
\text { on fundamental } \\
\text { movement skills (indoors } \\
\text { or outdoors), 40-50 } \\
\text { minutes each. Games and } \\
\text { activities developed for } \\
\text { this intervention focused } \\
\text { on mastery of six } \\
\text { fundamental movement } \\
\text { skills. IG } \text { students } \\
\text { participated in combined } \\
\text { intervention conditions } \\
\text { (behavioral and } \\
\text { fundamental movement } \\
\text { skill condition), receiving } \\
38 \text { intervention lessons. } \\
\text { CG: students received } \\
\text { usual school curriculum. }\end{array}$ & $\begin{array}{l}\text { 1.8. Behavioral contract } 2.3 \text {. } \\
\text { Self-monitoring of } \\
\text { behavior3.2. Social support } \\
\text { (practical) 4.1. Instruction } \\
\text { on how to perform the } \\
\text { behavior5.1. Information } \\
\text { about health } \\
\text { consequences6.1. } \\
\text { Demonstration of the } \\
\text { behavior } 8.1 \text {. Behavioral } \\
\text { practice/rehearsal8.2. } \\
\text { Behavior substitution }\end{array}$ & $\begin{array}{l}\text { 1. Goals and planning2. } \\
\text { Feedback and } \\
\text { monitoring3. Social } \\
\text { support4. Shaping } \\
\text { knowledge5. Natural } \\
\text { consequences6. } \\
\text { Comparison of } \\
\text { behavior8. Repetition } \\
\text { and substitution }\end{array}$ & enjoyment* & $\begin{array}{l}\text { counts/day** } \\
\text { (accelerometer) MPA (min/ } \\
\text { day) })^{* *} \text { VPA }(\min / \text { day) })^{* *}\end{array}$ \\
\hline $\begin{array}{l}\text { Shannon et al. } \\
\text { (2018);Northern } \\
\text { IrelandCT }\end{array}$ & $\begin{array}{l}\mathrm{N}=155 \text { children } \\
\text { ( } 44 \% \text { boys), age: } 8.7 \\
\text { ( } 0.5) \text { years. IG: } \\
\mathrm{n}=84 \text { ( } 33 \text { boys); CG: } \\
\mathrm{n}=71 \text { ( } 36 \text { boys). }\end{array}$ & SDT & $\begin{array}{l}\text { IG: over } 10 \text { weeks, } \\
\text { students participated in } \\
\text { the "Healthy Choice } \\
\text { Program" in addition to } \\
\text { regular PE. Weekly 60- } \\
\text { minute sessions were } \\
\text { delivered by a sport } \\
\text { student volunteer under } \\
\text { supervision of the } \\
\text { classroom teacher. } \\
\text { Additionally, teachers } \\
\text { conducted a 15-minute } \\
\text { walk with the students }\end{array}$ & $\begin{array}{l}\text { 1.1. Goal setting (behavior) } \\
\text { 1.4. Action planning2.2. } \\
\text { Feedback on behavior3.3. } \\
\text { Social support (emotional) } \\
\text { 5.1. Information about } \\
\text { health consequences }\end{array}$ & $\begin{array}{l}\text { 1. Goals and planning2. } \\
\text { Feedback and } \\
\text { monitoring3. Social } \\
\text { support5. Natural } \\
\text { consequences }\end{array}$ & $\begin{array}{l}\text { teacher autonomy support* autonomy"competence } \\
\text { relatednessintrinsic motivationidentified } \\
\text { regulationintrojected regulationexternal regulation }\end{array}$ & $\begin{array}{l}\text { school day MVPA (min/ } \\
\text { day) (accelerometer)* }\end{array}$ \\
\hline
\end{tabular}

\section{pehavior4.1. Instruction on monitoring4. Shaping \\ comparison of \\ the command teaching of the behavior8.1. behavior8. Repet}

attended 10 tennis lessons rehears

teaching style. CG:

students did not receive

1.8. Behavioral contract 2.3. 1. Goals and planning2. enjoyment

and sport

behavior3.2. Social

(accelerometer) MPA (min/

ay)** VPA $(\min /$ day $) * *$

(continued on next page) 
Table 1 (continued)

\begin{tabular}{|c|c|c|c|c|c|c|c|}
\hline $\begin{array}{l}\text { Author; } \\
\text { CountryDesign }\end{array}$ & Participants & $\begin{array}{l}\text { Theoretical } \\
\text { background/ } \\
\text { Pedagogical } \\
\text { approach }\end{array}$ & Intervention description & Behavior change techniques & $\begin{array}{l}\text { Behavior change } \\
\text { technique cluster }\end{array}$ & Motivational outcomes & Physical activity outcomes \\
\hline & & & $\begin{array}{l}\text { every school day. Sport } \\
\text { student volunteers and } \\
\text { classroom teachers } \\
\text { received a needs- } \\
\text { supportive teaching } \\
\text { training prior to the } \\
\text { intervention. CG: students } \\
\text { received intervention } \\
\text { after the posttest. }\end{array}$ & & & & \\
\hline $\begin{array}{l}\text { Smith et al. (2015); } \\
\text { EnglandCT }\end{array}$ & $\begin{array}{l}\mathrm{N}=72 \text { students ( } 42 \\
\text { boys), age boys: } \\
11.22 \text { ( } 0.4 \text { ) years, } \\
\text { age girls: } 11.4(0.5) \\
\text { years. IG: } \mathrm{n}=36(23 \\
\text { boys); CG: } \mathrm{n}=36 \text { (19 } \\
\text { boys) }\end{array}$ & $\begin{array}{l}\text { SDT Tactical } \\
\text { Games Model } \\
\text { (TGfU) }\end{array}$ & $\begin{array}{l}\text { IG: over } 12 \text { weeks, } \\
\text { students participated in } \\
\text { weekly PE classes } \\
\text { following the Tactical } \\
\text { Games Model teaching } \\
\text { approach. Girls } \\
\text { participated in activities } \\
\text { such as netball and } \\
\text { football. Boys } \\
\text { participated in rugby and } \\
\text { football. CG: students } \\
\text { participated in PE classes } \\
\text { taught using a traditional, } \\
\text { direct skill instruction } \\
\text { approach. }\end{array}$ & $\begin{array}{l}\text { 1.2. Problem solving4.1. } \\
\text { Instruction on how to } \\
\text { perform the behavior6.1. } \\
\text { Demonstration of the } \\
\text { behavior8.1. Behavioral } \\
\text { practice/rehearsal }\end{array}$ & $\begin{array}{l}\text { 1. Goals and planning } 4 . \\
\text { Shaping knowledge6. } \\
\text { Comparison of } \\
\text { behavior8. Repetition } \\
\text { and substitution }\end{array}$ & $\begin{array}{l}\text { autonomycompetencerelatednessintrinsic } \\
\text { motivationenjoyment }\end{array}$ & $\begin{array}{l}\text { girls' in-class MVPA (\%) } \\
\text { (observation) boys in-class } \\
\text { MVPA (\%)*girls' in-class } \\
\text { MVPA (accelerometer)** } \\
\text { boys' in-class MVPA*** }\end{array}$ \\
\hline $\begin{array}{l}\text { Todorovich \& } \\
\text { Curtner-Smith } \\
\text { (2002);USACT }\end{array}$ & $\begin{array}{l}\mathrm{N}=72(45 \text { boys) } 6 \text { th } \\
\text { grade students, age: } \\
11 \text { ( } 3.3 \text { years. } \mathrm{IG}_{1}: \\
\mathrm{n}=25 \text { ( } 16 \text { boys); } \mathrm{IG}_{2}: \\
\mathrm{n}=23 \text { ( } 13 \text { boys); CG: } \\
\mathrm{n}=24 \text { ( } 16 \text { boys). }\end{array}$ & AGTTARGET & $\begin{array}{l}\text { IG }_{1} \text { : over two weeks, } \\
\text { students participated in a } \\
\text { modified field hockey unit } \\
\text { within a high ego- } \\
\text { involving motivational } \\
\text { climate, Lessons were } \\
\text { held daily, } 30 \text { minute } \\
\text { each. IG } \mathrm{G}_{2} \text { participants } \\
\text { took part in a unit of } \\
\text { modified field hockey unit } \\
(10 \times 30 \text { minutes for two } \\
\text { weeks) within a high task- } \\
\text { involving motivational } \\
\text { climate. The components } \\
\text { of intervention lessons } \\
\text { were based on TARGET. } \\
\text { CG: a PE assistant taught } \\
10 \times 30 \text { minute lessons of } \\
\text { softball. }\end{array}$ & $\begin{array}{l}1.1 \text { Goal setting (behavior) } \\
\text { 2.3. Self-monitoring of } \\
\text { behavior2.7. Feedback on } \\
\text { outcome(s) of behavior4.1 } \\
\text { Instruction on how to } \\
\text { perform the behavior6.1. } \\
\text { Demonstration of the } \\
\text { behavior6.2. Social } \\
\text { comparison8.1. Behavioral } \\
\text { practice/rehearsal10.3. } \\
\text { Non-specific reward12.2. } \\
\text { Restructuring the social } \\
\text { environment }\end{array}$ & $\begin{array}{l}\text { 1. Goals and planning2. } \\
\text { Feedback and } \\
\text { monitoring4. Shaping } \\
\text { knowledge6. } \\
\text { Comparison of } \\
\text { behavior8. Repetition } \\
\text { and substitution10. } \\
\text { Reward and threat12. } \\
\text { Antecedents }\end{array}$ & ego orientation**task orientation $* *$ & $\mathrm{x}$ \\
\hline $\begin{array}{l}\text { Todorovich } \\
\text { \&Curtner-Smith } \\
\text { (2003);USACT }\end{array}$ & $\begin{array}{l}\mathrm{N}=80(40 \text { boys) } 3 \mathrm{rd} \\
\text { grade students. } \mathrm{IG}_{1}: \\
\mathrm{n}=28\left(15 \text { boys); } \mathrm{IG}_{2}:\right. \\
\mathrm{n}=26(13 \text { boys); } \mathrm{CG}: \\
\mathrm{n}=26(12 \text { boys }) .\end{array}$ & AGTTARGET & $\begin{array}{l}\mathrm{IG}_{1} \text { : students participated } \\
\text { in a modified field hockey } \\
\text { unit (10 lessons) within a } \\
\text { high task-involving } \\
\text { climate. The components } \\
\text { of the lessons were based } \\
\text { on TARGET. IG } \text { I }_{2} \text { students }\end{array}$ & $\begin{array}{l}\text { 1.1. Goal setting (behavior) } \\
\text { 2.3. Self-monitoring of } \\
\text { behavior2.7. Feedback on } \\
\text { outcome(s) of behavior4.1. } \\
\text { Instruction on how to } \\
\text { perform the behavior6.1. } \\
\text { Demonstration of the }\end{array}$ & $\begin{array}{l}\text { 1. Goals and planning2. } \\
\text { Feedback and } \\
\text { monitoring4. Shaping } \\
\text { knowledge6. } \\
\text { Comparison of } \\
\text { behavior8. Repetition } \\
\text { and substitution10. }\end{array}$ & task orientation**ego orientation $* *$ & $\mathrm{x}$ \\
\hline
\end{tabular}

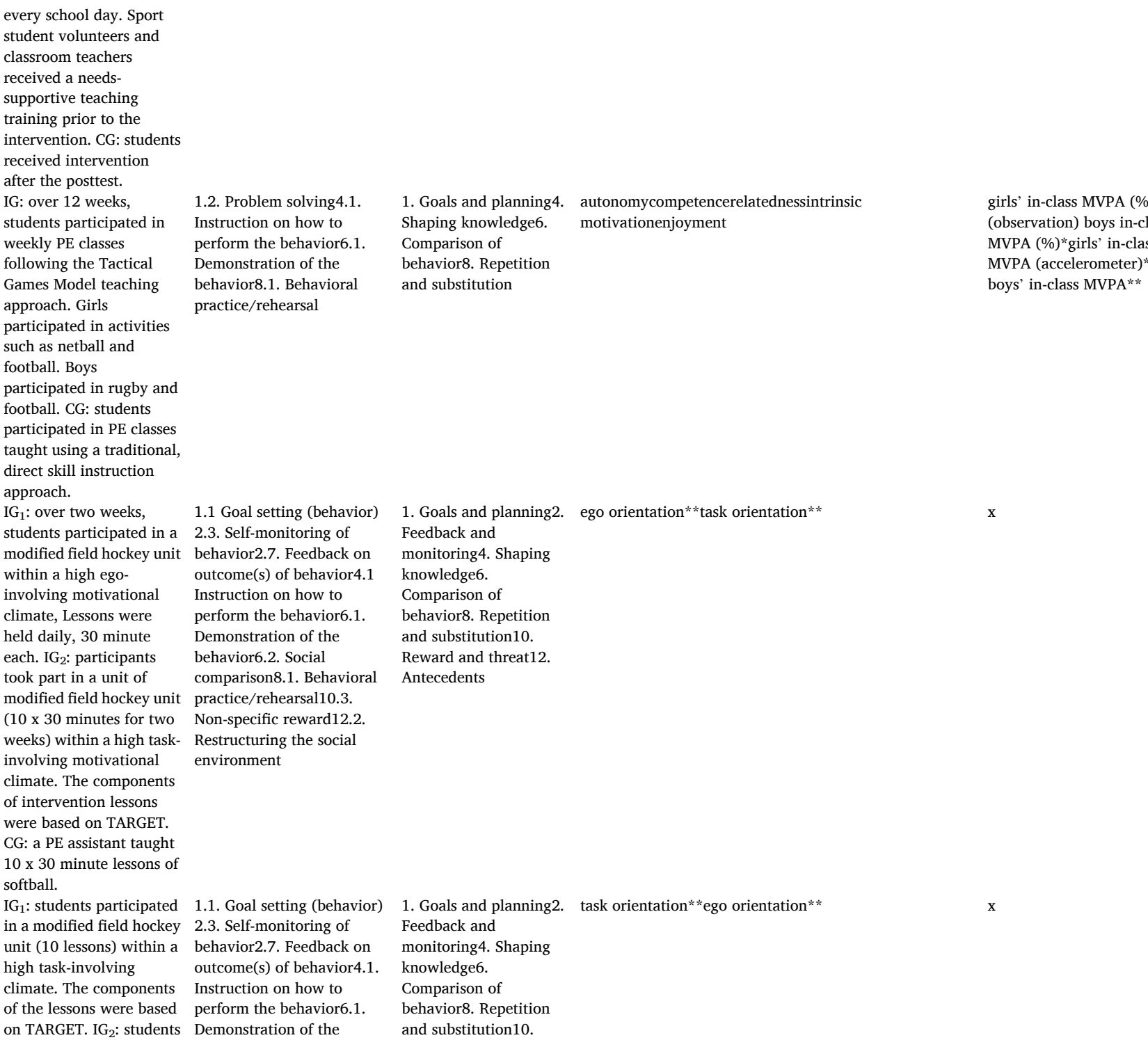

80 ( 40 boys) 3rd AGTTARGET

grade students. $\mathrm{IG}_{1}$

$\mathrm{n}=28$ (15 boys); $\mathrm{IG}_{2}$ :

$\mathrm{n}=26$ (12 boys). 


\section{Table 1 (continued)}

\begin{tabular}{|c|c|c|c|c|c|c|c|}
\hline $\begin{array}{l}\text { Author; } \\
\text { CountryDesign }\end{array}$ & Participants & $\begin{array}{l}\text { Theoretical } \\
\text { background/ } \\
\text { Pedagogical } \\
\text { approach }\end{array}$ & Intervention description & Behavior change techniques & $\begin{array}{l}\text { Behavior change } \\
\text { technique cluster }\end{array}$ & Motivational outcomes & Physical activity outcomes \\
\hline $\begin{array}{l}\text { Wang et al. (2017); } \\
\text { China } \\
\text { CT }\end{array}$ & $\begin{array}{l}\mathrm{N}=179 \text { primary } \\
\text { school students (103 } \\
\text { boys), age: } 10.2 \\
\text { years. IG: } \mathrm{n}=95 \text { ( } 57 \\
\text { boys); CG: } \mathrm{n}=84 \text { ( } 46 \\
\text { boys). }\end{array}$ & $\begin{array}{l}\text { SDT, Self-efficacy } \\
\text { theory }\end{array}$ & $\begin{array}{l}\text { participated in a 10-lesson } \\
\text { modified field hockey unit } \\
\text { within a high ego- } \\
\text { involving climate. CG: a } \\
\text { PE assistant taught } 10 \mathrm{x} \\
30 \text { minutes lessons of } \\
\text { softball. } \\
\text { IG: students played the } \\
\text { video game Diab, } \\
\text { designed based on social } \\
\text { cognitive, self- } \\
\text { determination, and } \\
\text { elaboration-likelihood } \\
\text { models regarding PA and } \\
\text { diet. Students played Diab } \\
\text { either at } 2 \times \text { x } 40-\text {-minute } \\
\text { morning sessions before } \\
\text { classes ( } 2 \text { schools) or at } \\
\text { one } 90 \text {-minute afternoon } \\
\text { session after school (in } \\
\text { other } 2 \text { schools). The } \\
\text { intervention lasted } 8 \text { to } 10 \\
\text { weeks, depending on } \\
\text { completion of the game (9 } \\
\text { episodes, with } 3 \text { to } 4 \text { mini- } \\
\text { games). CG: no } \\
\text { intervention, children } \\
\text { adopted general diet and } \\
\text { PA information and } \\
\text { behavior as usual. }\end{array}$ & $\begin{array}{l}\text { behavior6.2. Social } \\
\text { comparison8.1. Behavioral } \\
\text { practice/rehearsal10.3. } \\
\text { Non-specific reward12.2. } \\
\text { Restructuring the social } \\
\text { environment } \\
\text { 1.1. Goal setting (behavior) } \\
\text { 1.2. Problem solving } \\
\text { 1.4. Action planning } \\
\text { 1.5. Review behavior goal(s) } \\
\text { 2.2. Feedback on behavior } \\
\text { 5.1. Information about } \\
\text { health consequences } \\
\text { 10.1. Material incentive } \\
\text { (behavior) }\end{array}$ & $\begin{array}{l}\text { 1. Goals and planning } \\
\text { 2. Feedback and } \\
\text { monitoring } \\
\text { 5. Natural consequences } \\
\text { 10. Reward and threat }\end{array}$ & $\begin{array}{l}\text { autonomous motivation } \\
\text { controlled motivation }\end{array}$ & $\begin{array}{l}\text { self-reported PA* } \\
\text { sedentary time (min) } \\
\text { (accelerometer) } \\
\text { LPA (min) } \\
\text { MVPA (min) }\end{array}$ \\
\hline
\end{tabular}

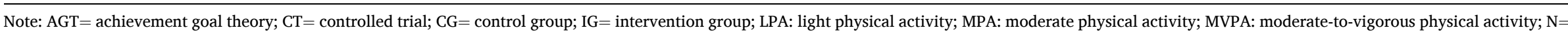

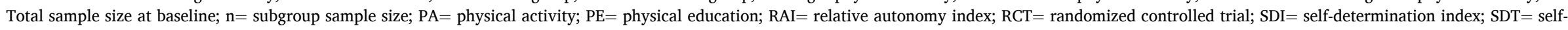
determination theory; TARGET $=$ Task, Authority, Recognition, Grouping, Evaluation, Time; VPA= vigorous PA.* indicates significant effects (pre to post) with a $p$-value $<0.05$; ** $p$-value $<0.01$. 
Table 2

Description of reviewed studies targeting adolescents with examined outcomes at a minimum of two time points (pre to post).

\begin{tabular}{|c|c|c|c|c|c|c|c|}
\hline $\begin{array}{l}\text { Author; } \\
\text { CountryDesign }\end{array}$ & Participants & $\begin{array}{l}\text { Theoretical } \\
\text { background/ } \\
\text { Pedagogical } \\
\text { model }\end{array}$ & Intervention description & $\begin{array}{l}\text { Behavior change } \\
\text { techniques }\end{array}$ & $\begin{array}{l}\text { Behavior change } \\
\text { technique clusters }\end{array}$ & Motivational outcomes & Physical activity outcomes \\
\hline $\begin{array}{l}\text { Barzouka et al. } \\
\text { (2015); GreeceCT }\end{array}$ & $\begin{array}{l}\mathrm{N}=63 \text { high school } \\
\text { students, age: } 13.1 \\
(0.89) \text { years. IG } \mathrm{I}_{1}: \\
\mathrm{n}=20 ; \mathrm{IG}_{2}: \mathrm{n}=21 ; \\
\text { CG: } \mathrm{n}=22 .\end{array}$ & $\begin{array}{l}\text { Social cognitive } \\
\text { theory/ } \\
\text { Observational } \\
\text { learning }\end{array}$ & $\begin{array}{l}\text { Over } 5 \text { weeks, students } \\
\text { received a PE intervention } \\
\text { twice a week for } 45 \\
\text { minutes. IG } 1 \text { : students } \\
\text { observed a videotaped } \\
\text { expert model, which was a } \\
\text { student/athlete the same } \\
\text { age of the participants } \\
\text { with experience in } \\
\text { volleyball, and received } \\
\text { verbal instructions from } \\
\text { PE teacher. IG } \text { I }_{2} \text { students } \\
\text { observed a videotaped } \\
\text { expert model plus their } \\
\text { own video recording (self- } \\
\text { monitoring) and verbal } \\
\text { instructions were given. } \\
\text { CG: students received } \\
\text { traditional feedback with } \\
\text { verbal instructions on } \\
\text { correct skill execution. }\end{array}$ & $\begin{array}{l}\text { 2.2. Feedback on } \\
\text { behavior2.4. Self- } \\
\text { monitoring of behavior } 4.1 \text {. } \\
\text { Instruction on how to } \\
\text { perform the behavior } 6.1 \text {. } \\
\text { Demonstration of the } \\
\text { behavior } 8.1 \text {. Behavioral } \\
\text { practice/rehearsal }\end{array}$ & $\begin{array}{l}\text { 2. Feedback and } \\
\text { monitoring } 4 \text {. Shaping } \\
\text { knowledge6. } \\
\text { Comparison of } \\
\text { behavior8. Repetition } \\
\text { and substitution }\end{array}$ & task goal orientation*ego goal orientation & $\mathrm{x}$ \\
\hline $\begin{array}{l}\text { Brankovic \& } \\
\text { Hadzikadunic } \\
\text { (2017); Bosnia \& } \\
\text { HerzegovinaCT }\end{array}$ & $\begin{array}{l}\mathrm{N}=74 \text { 8th grade } \\
\text { students ( } 40 \text { male), } \\
\text { age: } 13 \text { to } 15 \text { years. } \\
\text { IG: } \mathrm{n}=53 \text {; CG: } \\
\mathrm{n}=21 .\end{array}$ & $\begin{array}{l}\text { Flow: Psychology } \\
\text { of optimal } \\
\text { experience, SDT }\end{array}$ & $\begin{array}{l}\text { IG: students participated } \\
\text { in } 10 \text { PE lessons twice a } \\
\text { week, designed to ignite } \\
\text { perception of self- } \\
\text { competence (creative } \\
\text { thinking, self-potentials } \\
\text { and interests). Lessons } \\
\text { were held by the PE } \\
\text { teacher. CG: students } \\
\text { participated in activities of } \\
\text { traditional PE lesson. }\end{array}$ & $\begin{array}{l}\text { 2.2. Feedback on } \\
\text { behavior3.2. Social support } \\
\text { (practical)5.6. Information } \\
\text { about emotional } \\
\text { consequences12.2. } \\
\text { Restructuring the social } \\
\text { environment }\end{array}$ & $\begin{array}{l}\text { 2. Feedback and } \\
\text { monitoring3. Social } \\
\text { support5. Natural } \\
\text { consequences12. } \\
\text { Antecedents }\end{array}$ & competence & $\mathrm{x}$ \\
\hline \multirow[t]{2}{*}{$\begin{array}{l}\text { Bush et al. (2010); } \\
\text { CanadaCT }\end{array}$} & $\begin{array}{l}\mathrm{N}=221 \text { 7th-8th } \\
\text { grade students } \\
\text { (40.7\% boys). IG: } \\
\mathrm{n}=131 \text { ( } 62 \text { boys), } \\
\text { age boys: } 13.9 \\
\text { ( } 0.7) \text { years, age } \\
\text { girls: } 13.8(0.9) \\
\text { years; CG: } \mathrm{n}=90 \\
\text { (28 boys), age } \\
\text { boys:12.5 (0.6) } \\
\text { years, age } \\
\text { girls:12.5 (0.6) } \\
\text { years. }\end{array}$ & $\begin{array}{l}\text { participant } \\
\text { centered approach }\end{array}$ & $\begin{array}{l}\text { IG: over } 16 \text { weeks, } \\
\text { students participated in } \\
\text { the FunAction PA program } \\
3-5 \text { times a week for } 45 \\
\text { minutes. FunAction was } \\
\text { delivered during the first } \\
45 \text { minutes of lunch } \\
\text { period, held by university } \\
\text { students. Participants and } \\
\text { teachers were surveyed at } \\
\text { beginning and middle of } \\
\text { the program in order to } \\
\text { make modifications } \\
\text { according to their } \\
\text { preferences and reduce } \\
\text { barriers. CG: no } \\
\text { information on control } \\
\text { condition. }\end{array}$ & $\begin{array}{l}\text { 6.1. Demonstration of } \\
\text { behavior7.1. Prompts/ } \\
\text { Cues10.1. Material } \\
\text { Incentive (behavior)10.2. } \\
\text { Material reward (behavior) } \\
\text { 12.2. Restructuring the } \\
\text { social environment }\end{array}$ & $\begin{array}{l}\text { 6. Comparison of } \\
\text { behavior7. } \\
\text { Associations } 10 . \\
\text { Reward and threat } 12 \text {. } \\
\text { Antecedents }\end{array}$ & enjoyment & $\begin{array}{l}\text { self-reported leisure time } \\
\text { PA }\end{array}$ \\
\hline & & SDT & & & & & $\mathrm{x}$ \\
\hline
\end{tabular}




\begin{tabular}{|c|c|c|c|c|c|c|c|}
\hline $\begin{array}{l}\text { Author; } \\
\text { CountryDesign }\end{array}$ & Participants & $\begin{array}{l}\text { Theoretical } \\
\text { background/ } \\
\text { Pedagogical } \\
\text { model }\end{array}$ & Intervention description & $\begin{array}{l}\text { Behavior change } \\
\text { techniques }\end{array}$ & $\begin{array}{l}\text { Behavior change } \\
\text { technique clusters }\end{array}$ & Motivational outcomes & Physical activity outcomes \\
\hline $\begin{array}{l}\text { Chang et al. (2016); } \\
\text { TaiwanCT }\end{array}$ & $\begin{array}{l}\mathrm{N}=126 \text { 6th grade } \\
\text { students; age }=13.7 \\
\text { (0.5) years. IG: } \\
\mathrm{n}=61 \text { ( } 30 \text { boys); } \\
\text { CG: } \mathrm{n}=65 \text { ( } 35 \\
\text { boys) }\end{array}$ & & $\begin{array}{l}\text { IG: over } 6 \text { weeks, students } \\
\text { took part in autonomy } \\
\text { supportive PE classes } \\
\text { twice a week for } 40 \\
\text { minutes. Participants } \\
\text { decided on sequence of } \\
\text { content by consensus and } \\
\text { chose their partners. CG: } \\
\text { students took part in } \\
\text { identical PE content and } \\
\text { skill instructions, the } \\
\text { sequence of content was } \\
\text { determined by the teacher } \\
\text { and group partners were } \\
\text { assigned by the PE teacher } \\
\text { for each lesson. }\end{array}$ & $\begin{array}{l}\text { 4.1. Instruction on how to } \\
\text { perform6.1. Demonstration } \\
\text { of the behavior8.1. } \\
\text { Behavioral practice/ } \\
\text { rehearsal12.2. } \\
\text { Restructuring the social } \\
\text { environment }\end{array}$ & $\begin{array}{l}\text { 4. Shaping } \\
\text { knowledge6. } \\
\text { Comparison of } \\
\text { behavior8. Repetition } \\
\text { and substitution12. } \\
\text { Antecedents }\end{array}$ & $\begin{array}{l}\text { perceived teaching autonomy**autonomy in } \mathrm{PE}^{* *} \text { intrinsic } \\
\text { motivation*amotivationexternal motivationintrojected } \\
\text { regulationidentified regulation }\end{array}$ & \\
\hline $\begin{array}{l}\text { Chatzisarantis } \\
\text { \&Hagger (2009); } \\
\text { EnglandRCT }\end{array}$ & $\begin{array}{l}\mathrm{N}=215 \text { students } \\
\text { from } 10 \text { classes, } \\
\text { age: } 14.84(0.40) \\
\text { years. IG: } \mathrm{n}=5 \\
\text { classes; CG: } \mathrm{n}=5 \\
\text { classes. }\end{array}$ & SDT & $\begin{array}{l}\text { IG: over } 5 \text { weeks, teachers } \\
\text { adopted an autonomous } \\
\text { inter-personal teaching } \\
\text { style during PE. Teachers } \\
\text { provided positive } \\
\text { feedback, rationale and } \\
\text { choice and acknowledged } \\
\text { difficulties associated with } \\
\text { PE. CG: teachers used a } \\
\text { less autonomy supportive } \\
\text { instruction style providing } \\
\text { rationale and feedback } \\
\text { only. }\end{array}$ & $\begin{array}{l}\text { 2.2. Feedback on } \\
\text { behavior5.1. Information } \\
\text { about health } \\
\text { consequences5.6. } \\
\text { Information about } \\
\text { emotional consequences }\end{array}$ & $\begin{array}{l}\text { 2. Feedback and } \\
\text { monitoring5. Natural } \\
\text { consequences }\end{array}$ & teacher autonomy support**RAI* & $\mathrm{x}$ \\
\hline $\begin{array}{l}\text { Chiva-Bartol et al. } \\
\text { (2018);SpainCT }\end{array}$ & $\begin{array}{l}\mathrm{N}=96 \text { secondary } \\
\text { school students ( } 46 \\
\text { boys), age: } 15(0.7) \\
\text { years. IG: } \mathrm{n}=31 \text { (15 } \\
\text { boys), age: } 15(0.4) \\
\text { years; CG: } \mathrm{n}=65 \\
\text { (31 boys), age: } 15 \\
\text { (0.6) years. }\end{array}$ & $\begin{array}{l}\text { AGT, SDT/ } \\
\text { Teaching Games } \\
\text { for Understanding } \\
\text { (TGfU), } \\
\text { Cooperative } \\
\text { Learning }\end{array}$ & $\begin{array}{l}\text { IG: over } 8 \text { weeks, students } \\
\text { participated in a handball } \\
\text { teaching unit in } \\
\text { accordance with the } \\
\text { Cooperative Learning and } \\
\text { TGfU approaches (hybrid } \\
\text { approach). Lessons were } \\
\text { held twice a week for 55- } \\
60 \text { minutes. CG: students } \\
\text { participated in a teaching } \\
\text { unit according to the } \\
\text { traditional teaching } \\
\text { approach. }\end{array}$ & $\begin{array}{l}\text { 1.2. Problem solving2.2. } \\
\text { Feedback on behavior; } 4.1 \text {. } \\
\text { Instruction on how to } \\
\text { perform the behavior6.1. } \\
\text { Demonstration of the } \\
\text { behavior8.1. Behavioral } \\
\text { practice/rehearsal12.2. } \\
\text { Restructuring the social } \\
\text { environment13.1 } \\
\text { Identification of self as role } \\
\text { model }\end{array}$ & $\begin{array}{l}\text { 1. Goals and planning2. } \\
\text { Feedback and } \\
\text { monitoring4. Shaping } \\
\text { knowledge6. } \\
\text { Comparison of } \\
\text { behavior8. Repetition } \\
\text { and substitution12. } \\
\text { Antecedents13. } \\
\text { Identity }\end{array}$ & $\begin{array}{l}\text { global motivational climate scoretask-involving climate**ego- } \\
\text { involving climate** }\end{array}$ & $\mathrm{x}$ \\
\hline $\begin{array}{l}\text { Christodoulidis et al. } \\
\text { (2001);GreeceCT }\end{array}$ & $\begin{array}{l}\mathrm{N}=634 \text { 10th grade } \\
\text { students, age: } 16 \\
\text { (0.5) years. IG: } \\
\mathrm{n}=105 \text { ( } 47 \text { boys); } \\
\text { CG: } \mathrm{n}=529 \text { ( } 189 \\
\text { boys). }\end{array}$ & AGT & $\begin{array}{l}\text { IG: over one academic } \\
\text { year, } 25 \text { developed lesson } \\
\text { plans were implemented. } \\
\text { Teachers used different } \\
\text { teaching styles: practice } \\
\text { teaching style, inclusion } \\
\text { style and reciprocal } \\
\text { teaching style. Goal } \\
\text { oriented activities were } \\
\text { used, cooperative }\end{array}$ & $\begin{array}{l}\text { 1.3. Goal setting (outcome) } \\
\text { 2.4. Self-monitoring of } \\
\text { outcome(s) of behavior4.1. } \\
\text { Instruction on how to } \\
\text { perform the behavior5.1. } \\
\text { Information about health } \\
\text { consequences 6.1. } \\
\text { Demonstration of the } \\
\text { behavior 7.1. Prompts/ } \\
\text { Cues8.1. Behavioral }\end{array}$ & $\begin{array}{l}\text { 1. Goals and planning2. } \\
\text { Feedback and } \\
\text { monitoring4. Shaping } \\
\text { knowledge5. Natural } \\
\text { consequences6. } \\
\text { Comparison of } \\
\text { behavior7. } \\
\text { Associations8. } \\
\text { Repetition and }\end{array}$ & $\begin{array}{l}\text { teachers' emphasis on task-involvement*teachers' emphasis } \\
\text { on ego-involvement**ego goal orientationtask goal } \\
\text { orientationenjoyment }\end{array}$ & $\begin{array}{l}\text { self-reported duration/time } \\
\text { of sport/exercise } \\
\text { participation**self- } \\
\text { reported frequency of } \\
\text { sport/exercise involvement }\end{array}$ \\
\hline
\end{tabular}




\begin{tabular}{|c|c|c|c|c|c|c|c|}
\hline $\begin{array}{l}\text { Author; } \\
\text { CountryDesign }\end{array}$ & Participants & $\begin{array}{l}\text { Theoretical } \\
\text { background/ } \\
\text { Pedagogical } \\
\text { model }\end{array}$ & Intervention description & $\begin{array}{l}\text { Behavior change } \\
\text { techniques }\end{array}$ & $\begin{array}{l}\text { Behavior change } \\
\text { technique clusters }\end{array}$ & Motivational outcomes & Physical activity outcomes \\
\hline & & & $\begin{array}{l}\text { activities preferred over } \\
\text { competitive activities, and } \\
\text { an individualized goal- } \\
\text { setting program was } \\
\text { applied: students } \\
\text { documented their } \\
\text { performance and set } \\
\text { personal improvement } \\
\text { goals. Health related } \\
\text { information was provided } \\
\text { through short lectures on } \\
\text { positive effects of PA on } \\
\text { health. CG: students } \\
\text { participated in regular PE. }\end{array}$ & $\begin{array}{l}\text { practice/rehearsal12.2. } \\
\text { Restructuring the social } \\
\text { environment }\end{array}$ & $\begin{array}{l}\text { substitution } 12 . \\
\text { Antecedents }\end{array}$ & & \\
\hline $\begin{array}{l}\text { Cuevas et al. (2016); } \\
\text { SpainCT }\end{array}$ & $\begin{array}{l}\mathrm{N}=86 \text { secondary } \\
\text { school students ( } 37 \\
\text { boys), age: } 15.65 \\
\text { ( } 0.78) \text { years. IG: } \\
\mathrm{n}=43 \text {; CG: } \mathrm{n}=43 \text {. }\end{array}$ & $\begin{array}{l}\text { SDT/Sport } \\
\text { Education Model }\end{array}$ & $\begin{array}{l}\text { IG: over } 3 \text { months, } \\
\text { students participated in } 19 \\
\text { teaching units of } \\
\text { volleyball in accordance } \\
\text { with the Sport Education } \\
\text { Model twice a week for } 55 \\
\text { minutes. The model } \\
\text { introduces several } \\
\text { elements of the individual } \\
\text { sport in the units of PE } \\
\text { learning (preseason, } \\
\text { regular competition, and } \\
\text { final championship; } \\
\text { regular teams; final party; } \\
\text { etc.). Students } \\
\text { experienced different roles } \\
\text { in team sports and games } \\
\text { (referee, coach, player, } \\
\text { etc.). CG: students took } \\
\text { part in } 19 \text { units of } \\
\text { volleyball following the } \\
\text { traditional teaching } \\
\text { model. }\end{array}$ & $\begin{array}{l}\text { 4.1. Instruction on how to } \\
\text { perform the behavior6.1. } \\
\text { Demonstration of the } \\
\text { behavior6.2. Social } \\
\text { comparison8.1. Behavioral } \\
\text { practice/rehearsal10.4. } \\
\text { Social reward12.2. } \\
\text { Restructuring the social } \\
\text { environment }\end{array}$ & $\begin{array}{l}\text { 4. Shaping } \\
\text { knowledge6. } \\
\text { Comparison of } \\
\text { behavior8. Repetition } \\
\text { and substitution10. } \\
\text { Reward and threat12. } \\
\text { Antecedents }\end{array}$ & $\begin{array}{l}\text { intrinsic motivation*identified regulationintrojected } \\
\text { regulationexternal regulationamotivationSDIthwarting of } \\
\text { autonomythwarting competencethwarting } \\
\text { relatednesssatisfaction-enjoyment }\end{array}$ & 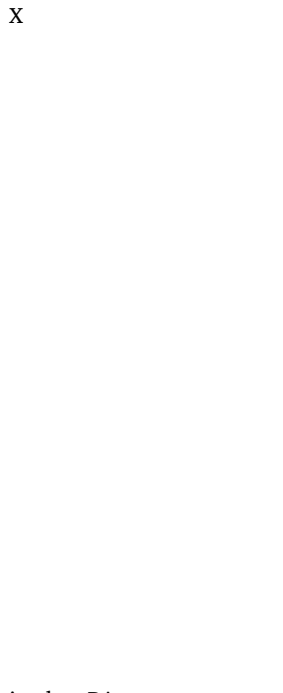 \\
\hline $\begin{array}{l}\text { Dudley et al. (2010); } \\
\text { AustraliaRCT }\end{array}$ & $\begin{array}{l}\mathrm{N}=38 \text { year } 11 \\
\text { female students. } \\
\text { IG: } \mathrm{n}=17 \text {, age: } \\
\text { 16.45 }(0.22) \text { years; } \\
\text { CG: } \mathrm{n}=21 \text {, age: } \\
16.57(0.28) \text { years. }\end{array}$ & $\begin{array}{l}\text { Social Cognitive } \\
\text { Theory }\end{array}$ & $\begin{array}{l}\text { IG: over } 11 \text { weeks, } \\
\text { students participated in six } \\
90 \text { minute PE sessions, } \\
\text { which included enjoyable, } \\
\text { challenging, and new } \\
\text { activities such as yoga/ } \\
\text { pilates/dance sessions, an } \\
\text { introductory tennis- } \\
\text { coaching course, and } \\
\text { aquatic games. Activities } \\
\text { were discussed with } \\
\text { participants prior and } \\
\text { during the intervention. } \\
\text { CG: continued with the } \\
\text { existing sport program. }\end{array}$ & $\begin{array}{l}3.2 \text { Social support } \\
\text { (practical)4.1. Instruction } \\
\text { on how to perform the } \\
\text { behavior5.6 Information } \\
\text { about emotional } \\
\text { consequences 6.1. } \\
\text { Demonstration of the } \\
\text { behavior8.1. Behavioral } \\
\text { practice/rehearsal12.1. } \\
\text { Restructuring physical } \\
\text { environment }\end{array}$ & $\begin{array}{l}\text { 3. Social support4. } \\
\text { Shaping knowledge5. } \\
\text { Natural consequences6. } \\
\text { Comparison of } \\
\text { behavior8. Repetition } \\
\text { and substitution12. } \\
\text { Antecedents }\end{array}$ & enjoymentsporting competencestrength competence & $\begin{array}{l}\text { in-class PA counts } \\
\text { (accelerometer) }\end{array}$ \\
\hline
\end{tabular}

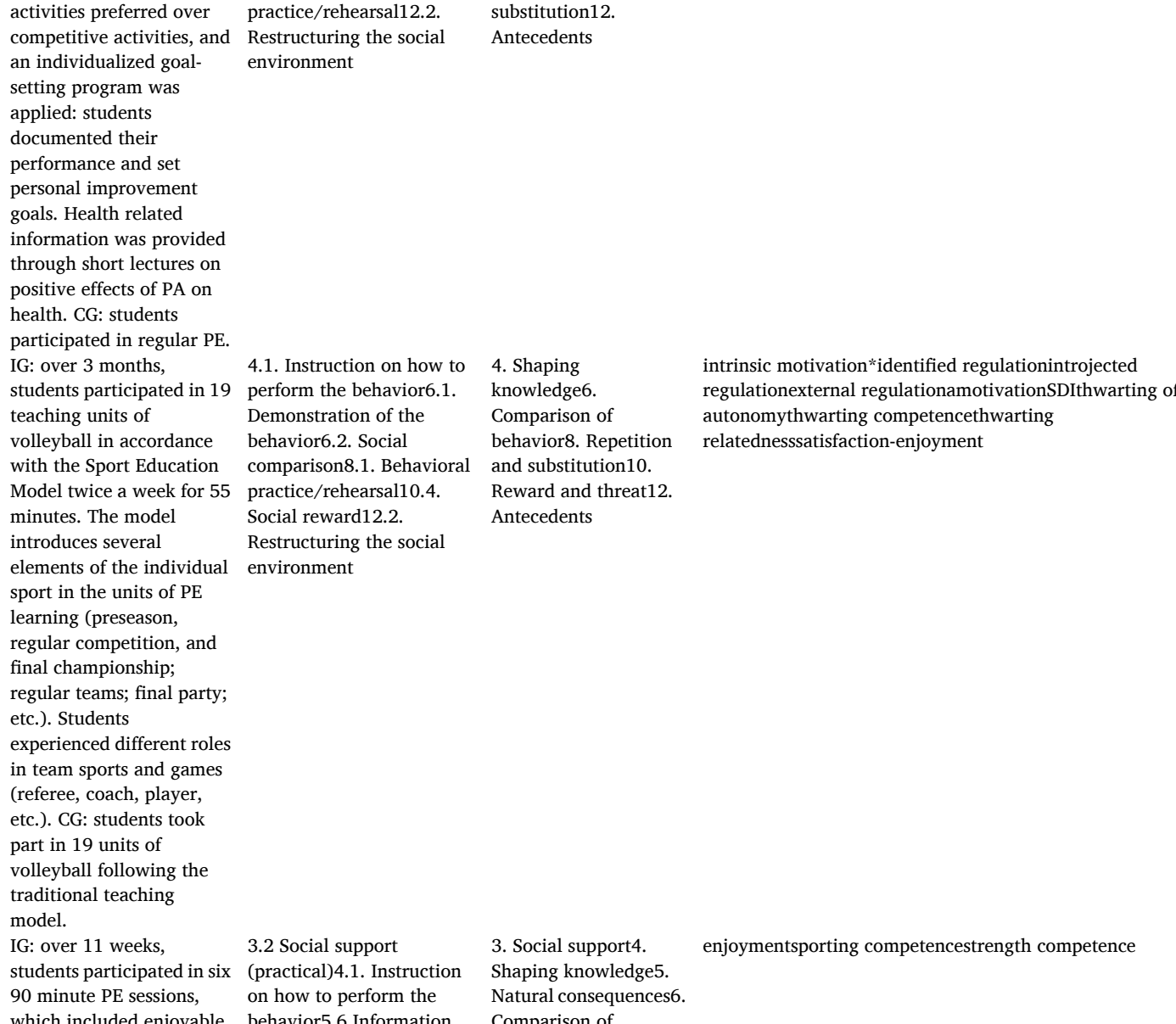




\begin{tabular}{|c|c|c|c|c|c|c|c|}
\hline $\begin{array}{l}\text { Author; } \\
\text { CountryDesign }\end{array}$ & Participants & $\begin{array}{l}\text { Theoretical } \\
\text { background/ } \\
\text { Pedagogical } \\
\text { model }\end{array}$ & Intervention description & $\begin{array}{l}\text { Behavior change } \\
\text { techniques }\end{array}$ & $\begin{array}{l}\text { Behavior change } \\
\text { technique clusters }\end{array}$ & Motivational outcomes & Physical activity outcomes \\
\hline $\begin{array}{l}\text { Franco \& Coterón } \\
\text { (2017);SpainCT }\end{array}$ & $\begin{array}{l}\mathrm{N}=53 \text { secondary } \\
\text { school students (30 } \\
\text { boys), age: } 13.35 \\
\text { (0.62) years. IG: } \\
\mathrm{n}=30 \text {; CG: } \mathrm{n}=23 \text {. }\end{array}$ & SDT & $\begin{array}{l}\text { IG: students participated } \\
\text { in } 24 \text { PE sessions of rugby } \\
\text { in accordance with a need } \\
\text { supportive environment. } \\
\text { The teacher was trained to } \\
\text { appropriately implement } \\
\text { the teaching strategies. } \\
\text { CG: students participated } \\
\text { in regular PE classes } \\
\text { (rugby) taught by the } \\
\text { school teacher. PE classes } \\
\text { were held twice a week. }\end{array}$ & $\begin{array}{l}\text { 2.2. Feedback on } \\
\text { behavior3.1. Social support } \\
\text { (unspecified) }\end{array}$ & $\begin{array}{l}\text { 2. Feedback and } \\
\text { monitoring3. Social } \\
\text { support }\end{array}$ & autonomy*competencerelatednessintrinsic motivation & $\mathrm{x}$ \\
\hline $\begin{array}{l}\text { Fu et al. (2013); } \\
\text { USACT }\end{array}$ & $\begin{array}{l}\mathrm{N}=61 \text { middle } \\
\text { school students }(25 \\
\text { boys), age: } 12.6 \\
\text { (0.6) years. IG: } \\
\mathrm{n}=31 \text { (12 boys); } \\
\text { CG: } \mathrm{n}=30 \text { (13 } \\
\text { boys) }\end{array}$ & $\begin{array}{l}\text { No information } \\
\text { provided }\end{array}$ & $\begin{array}{l}\text { IG: over } 6 \text { weeks, students } \\
\text { participated in health- } \\
\text { related physical fitness } \\
\text { basketball units offered } \\
\text { weekly for } 50 \text { minutes. } \\
\text { Lessons were implemented } \\
\text { according to the SPARK } \\
\text { Program (Sports, Play and } \\
\text { Active Recreation for Kids } \\
\text { Program). The teacher was } \\
\text { trained to use SPARK. } \\
\text { Most decisions on choice } \\
\text { of tasks, team structure, } \\
\text { and rate of progression } \\
\text { were determined by the } \\
\text { teacher. CG: students } \\
\text { participated in traditional } \\
\text { PE classes. }\end{array}$ & $\begin{array}{l}\text { 4.1. Instruction on how to } \\
\text { perform the behavior6.1. } \\
\text { Demonstration of the } \\
\text { behavior8.1. Behavioral } \\
\text { practice/rehearsal12.2. } \\
\text { Restructuring the social } \\
\text { environment }\end{array}$ & $\begin{array}{l}\text { 4. Shaping } \\
\text { knowledge6. } \\
\text { Comparison of } \\
\text { behavior8. Repetition } \\
\text { and substitution12. } \\
\text { Antecedents }\end{array}$ & enjoyment $*$ competence & in-class PA (pedometer) \\
\hline $\begin{array}{l}\text { Fu et al. (2016); } \\
\text { USACT }\end{array}$ & $\begin{array}{l}\mathrm{N}=174 \text { middle- } \\
\text { school students ( } 82 \\
\text { boys), age: } 12.06 \\
(0.85) \text { years. }\end{array}$ & $\begin{array}{l}\text { No information } \\
\text { provided }\end{array}$ & $\begin{array}{l}\text { IG: over } 9 \text { weeks students } \\
\text { participated in PE classes } \\
\text { in accordance with the } \\
\text { SPARK Program (Sports, } \\
\text { Play and Active Recreation } \\
\text { for Kids Program). Lessons } \\
\text { were offered weekly and } \\
\text { lasted approximately } 40 \\
\text { minutes. CG: students } \\
\text { participated in traditional } \\
\text { PE. }\end{array}$ & No information provided & $\begin{array}{l}\text { No information } \\
\text { provided }\end{array}$ & competenceenjoyment & $\begin{array}{l}\text { boys' in-class PA } \\
\text { (pedometer)** girls' in- } \\
\text { class } \text { PA** }^{* *}\end{array}$ \\
\hline $\begin{array}{l}\text { Gonzalez-Cutre et al. } \\
\text { (2018);SpainCT }\end{array}$ & $\begin{array}{l}\mathrm{N}=88 \text { ( } 36 \text { boys) } \\
\text { aged } 14-17 \text { years, } \\
\text { mean age: } 14.67 \\
(0.66) \text {; IG: } \mathrm{n}=29 \\
(15 \text { boys); CG: } \\
\mathrm{n}=59 \text { ( } 21 \text { boys). }\end{array}$ & SDT & $\begin{array}{l}\text { IG: over } 6 \text { months, } \\
\text { students participated in a } \\
\text { PE intervention with } 3 \\
\text { components: A) fitness and } \\
\text { health teaching unit of } 15 \\
\text { one-hour PE classes taught } \\
\text { by PE teacher trained in } \\
\text { motivational strategies to } \\
\text { satisfy basic psychological } \\
\text { needs. Lessons were held } \\
\text { twice a week for } 8 \text { weeks. }\end{array}$ & $\begin{array}{l}\text { 1.1. Goal setting (behavior) } \\
\text { 2.7. Feedback on outcome } \\
\text { (s) of behavior3.2. Social } \\
\text { support (practical) } 4.1 \text {. } \\
\text { Instruction on how to } \\
\text { perform the behavior5.1. } \\
\text { Information about health } \\
\text { consequences 6.1. } \\
\text { Demonstration of the } \\
\text { behavior8.1. Behavioral } \\
\text { practice/ rehearsal12.2. }\end{array}$ & $\begin{array}{l}\text { 1. Goals and planning2. } \\
\text { Feedback and } \\
\text { monitoring3. Social } \\
\text { support4. Shaping } \\
\text { knowledge5. Natural } \\
\text { consequences6. } \\
\text { Comparison of } \\
\text { behavior8. Repetition } \\
\text { and substitution12. } \\
\text { Antecedents }\end{array}$ & $\begin{array}{l}\text { after completion of teaching unit:teacher autonomy support } \\
\text { autonomy in } \mathrm{PE} / \text { leisure-time } \mathrm{PA} \mathrm{A}^{*} \text { competence in } \mathrm{PE} / \text { leisure- } \\
\text { time PArelatedness in } \mathrm{PE} / \text { leisure-time PAintrinsic motivation } \\
\text { in PE/leisure-time PA**integrated regulation in PE/leisure- } \\
\text { time PAidentified regulation in PE/leisure-time PAintrojected } \\
\text { regulation in PE/leisure-time PAexternal regulation in } \mathrm{PE}^{* *} \\
\text { leisure-time PAamotivation in } \mathrm{PE} * \text { leisure-time PA }\end{array}$ & $\begin{array}{l}\text { after completion of } \\
\text { teaching unit:self-reported } \\
\text { PA (min/day) } \\
\text { LPAMPA**hard PAvery } \\
\text { hard PA }\end{array}$ \\
\hline
\end{tabular}

$$
\begin{aligned}
& \text { IG: students participated } \\
& \text { in } 24 \text { PE sessions of rugby } \\
& \text { in accordance with a need } \\
& \text { supportive environment. } \\
& \text { The teacher was trained to } \\
& \text { appropriately implement } \\
& \text { the teaching strategies. } \\
& \text { CG: students participated } \\
& \text { in regular PE classes } \\
& \text { (rugby) taught by the } \\
& \text { school teacher. PE classes } \\
& \text { were held twice a week. }
\end{aligned}
$$

24 PE sessions of rugby behavior3.1. Social support monitoring3. Social

IG: over 6 weeks, students

related physical fitnes

boys), age: 12.6

Comparison of

eekly for 50 minutes.

Lessoms were in lemented

Antecedents

rogram (Sports, Play an

Active Recreation for Kids

trained to use SPARK.

Most decisions on choice

were determined by the

PE classes.

participated in PE classes

in accordance with the

Program (Sports,

for Kids Program). Lesson

were offered weekly and

participated in traditional

1.1. Goal setting (behavior) 1. Goals and planning2.

after completion of

$\mathrm{PE}$ intervention with $3 \quad$ (s) of

consequences6.

twice a week for 8 weeks. practice/ rehearsal12.2. 


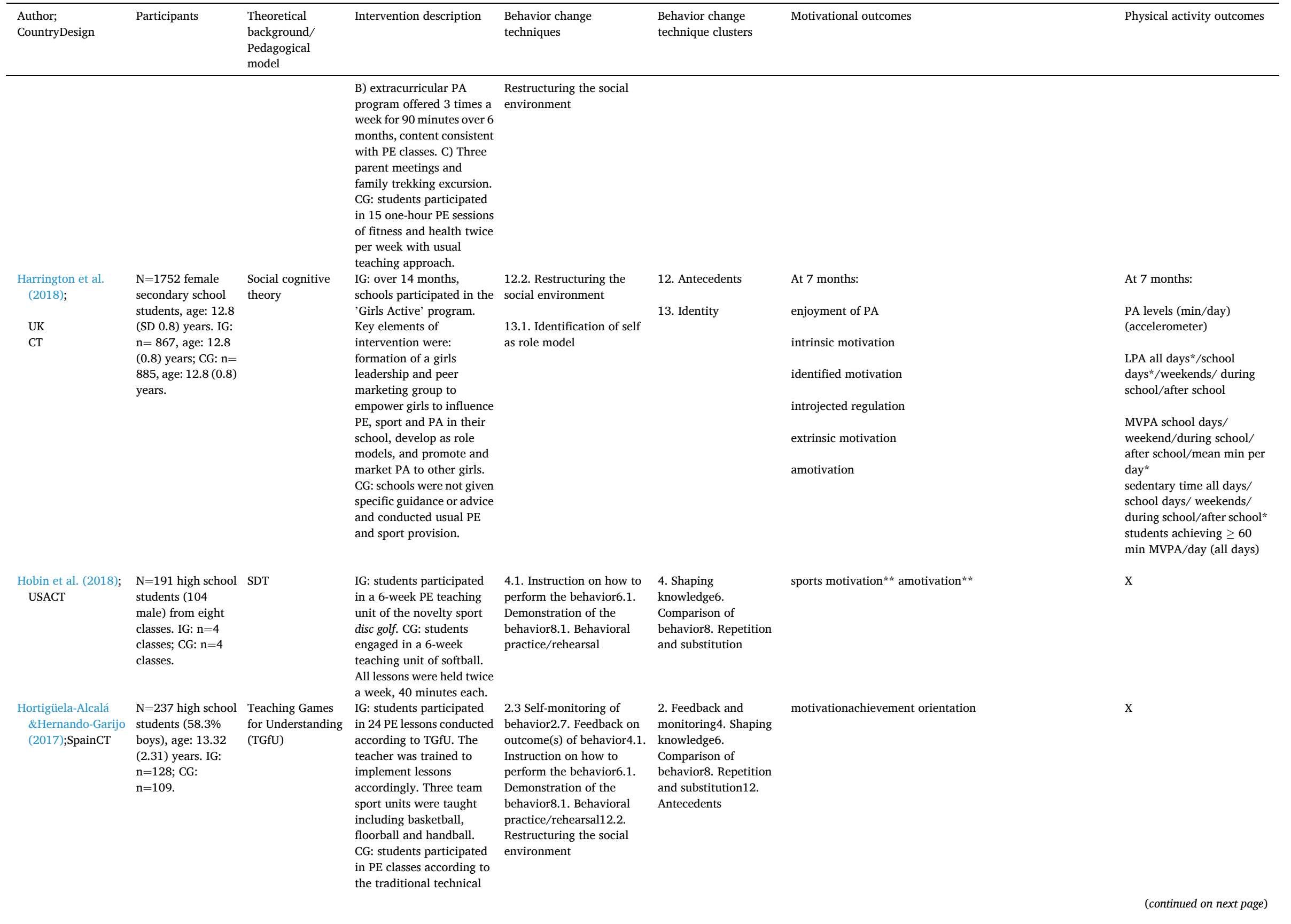

\begin{abstract}
B) extracurricular PA
program offered 3 times a
week for 90 minutes over 6

months, content consisten

with PE classes. C) Thr

family trekking excursion.

CG: students participated

of fitness and health twice

per week with usual

IG: over 14 months,
\end{abstract}

'Girls Active' program.

Key elements of

PA levels (min/day)

(accelerometer)

all days*/school

MVPA school days week PE teaching 40 minutes each.

ents participate

ortigüela-Alcalá $\quad \mathrm{N}=237$ high school Teaching Games Hernando-Garijo boys), age: 13.32 (TGfU) teacher was trained inplement lessons port units were taught loorball and handball.

PE classes according to 


\begin{tabular}{|c|c|c|c|c|c|c|c|}
\hline $\begin{array}{l}\text { Author; } \\
\text { CountryDesign }\end{array}$ & Participants & $\begin{array}{l}\text { Theoretical } \\
\text { background/ } \\
\text { Pedagogical } \\
\text { model }\end{array}$ & Intervention description & $\begin{array}{l}\text { Behavior change } \\
\text { techniques }\end{array}$ & $\begin{array}{l}\text { Behavior change } \\
\text { technique clusters }\end{array}$ & Motivational outcomes & Physical activity outcomes \\
\hline & & & $\begin{array}{l}\text { approach. Each lesson was } \\
45 \text { minutes long. }\end{array}$ & & & & \\
\hline $\begin{array}{l}\text { Jamner et al. (2004); } \\
\text { USACT }\end{array}$ & $\begin{array}{l}\mathrm{N}=47 \text { 10th and } \\
\text { 11th grade female } \\
\text { students } \\
\text { (sedentary), age: } \\
\text { 14.94 (0.79) years. } \\
\text { IG: } \mathrm{n}=25 \text {; CG: } \\
\mathrm{n}=22 .\end{array}$ & $\begin{array}{l}\text { No information } \\
\text { provided }\end{array}$ & $\begin{array}{l}\text { IG: over } 4 \text { months, } \\
\text { students took part in } \\
\text { special PE classes held } \\
\text { daily for } 60 \text { minutes. } \\
\text { Activities were selected } \\
\text { based on decision of focus } \\
\text { groups with members of } \\
\text { target population: aerobic } \\
\text { dance, basketball, } \\
\text { swimming and Tae Bo. A } \\
\text { weekly lecture was held on } \\
\text { health benefits of PA and } \\
\text { strategies to become more } \\
\text { active. Students were } \\
\text { exempted from PE uniform } \\
\text { requirement and periodic } \\
\text { 1-mile run test } \\
\text { administered in usual PE. } \\
\text { Classes were led by PE } \\
\text { teacher or a hired } \\
\text { instructor. CG: no } \\
\text { information. }\end{array}$ & $\begin{array}{l}\text { 1.1 Goal setting (behavior) } \\
\text { 1.2. Problem solving2.3. } \\
\text { Self-monitoring of behavior } \\
\text { 5.1. Information about } \\
\text { health consequences }\end{array}$ & $\begin{array}{l}\text { 1. Goals and planning2. } \\
\text { Feedback and } \\
\text { monitoring5. Natural } \\
\text { consequences }\end{array}$ & 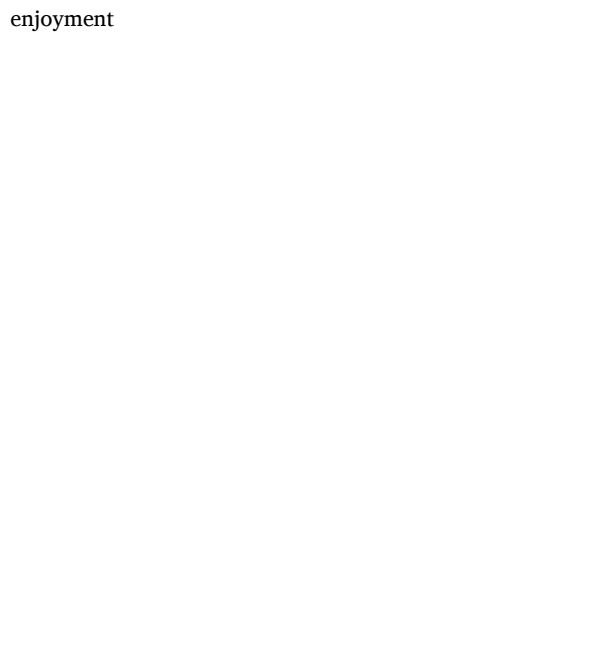 & $\begin{array}{l}\text { self-reported LPA*self- } \\
\text { reported MPA**self- } \\
\text { reported total PA*self- } \\
\text { reported lifestyle activity** }\end{array}$ \\
\hline $\begin{array}{l}\text { Jones et al. (2010); } \\
\text { EnglandCT }\end{array}$ & $\begin{array}{l}\mathrm{N}=202 \text { secondary } \\
\text { school students }(99 \\
\text { boys), age: } 11-14 \\
\text { years. }\end{array}$ & $\begin{array}{l}\text { Teaching Games } \\
\text { for Understanding } \\
\text { (TGfU) }\end{array}$ & $\begin{array}{l}\text { IG: over } 6 \text { weeks, teachers } \\
\text { implemented the TGfU } \\
\text { model, corresponding to } \\
\text { one unit of work of game } \\
\text { invasion. CG: students } \\
\text { participated in traditional, } \\
\text { skill-based PE lessons. }\end{array}$ & No information provided & $\begin{array}{l}\text { No information } \\
\text { provided }\end{array}$ & interest/enjoyment**choice/autonomy*** competence & $\mathrm{x}$ \\
\hline $\begin{array}{l}\text { Lonsdale et al. } \\
\text { (2013); } \\
\text { AustraliaRCT }\end{array}$ & $\begin{array}{l}\mathrm{N}=288 \text { year } 8 \\
\text { students from } 16 \\
\text { classes (50.4\% } \\
\text { boys, age: } 13.6 \\
\text { years. } \mathrm{IG}_{1}: \mathrm{n}=4 \\
\text { classes, } 60 \\
\text { students; } \mathrm{IG}_{2}: \mathrm{n}=4 \\
\text { classes, } 77 \\
\text { students; } \mathrm{IG}_{3}: \mathrm{n}=4 \\
\text { classes, } 71 \\
\text { students; CG: } \mathrm{n}=4 \\
\text { classes, } 80 \text { students }\end{array}$ & SDT & $\begin{array}{l}\text { Within } 3 \text { months, three IGs } \\
\text { participated in PE with } \\
\text { different intervention } \\
\text { strategies. IG } \mathrm{G}_{1} \text { : } \\
\text { 'Relevance', explaining } \\
\text { rationale and importance } \\
\text { of activities to students' } \\
\text { lives; IG }{ }_{2} \text { : 'Providing } \\
\text { choice', students had 2-4 } \\
\text { opportunities of choice } \\
\text { within lessons; IG } \mathrm{I}_{3} \text { :'Free } \\
\text { choice', providing } \\
\text { students with equipment, } \\
\text { but refraining from giving } \\
\text { instructions. Teachers } \\
\text { were given examples, such } \\
\text { as a number of options for } \\
\text { warm-up activities and } \\
\text { organizing two games near } \\
\text { end of the lesson -one } \\
\text { 'competitive' game with }\end{array}$ & $\begin{array}{l}\text { 5.1. Information about } \\
\text { health consequences5.6. } \\
\text { Information about } \\
\text { emotional } \\
\text { consequences6.2. Social } \\
\text { comparison12.2. } \\
\text { Restructuring the social } \\
\text { environment12.5. Adding } \\
\text { objects to the environment }\end{array}$ & $\begin{array}{l}\text { 5. Natural } \\
\text { consequences6. } \\
\text { Comparison of } \\
\text { behavior12. } \\
\text { Antecedents }\end{array}$ & $\begin{array}{l}\text { SDIautonomy***ompetence*relatednessteacher autonomy } \\
\text { support (provided choice)** }\end{array}$ & $\begin{array}{l}\text { in-class MVPA } \\
\text { (accelerometer)*in-class } \\
\text { sedentary time*** }\end{array}$ \\
\hline
\end{tabular}

\section{approach. Each lesson was}

IG: over 4 months,

students

(sedentary), age:

IG: $n=25$; CG:

elf-monitoring of behavior monitoring5. Natur

based on decision of focus

ups with members of

dance, basketball,

health benefits of PA and

pted from PE uniform

-mile run test

implemented the TGfU

invasion. CG: students

ehavior12

oys, age: 13.6

classes, 60

students; IG $\mathrm{I}: \mathrm{n}=4$

students; CG: $\mathrm{n}=4$

classes, 80 student 


\begin{tabular}{|c|c|c|c|c|c|c|c|}
\hline $\begin{array}{l}\text { Author; } \\
\text { CountryDesign }\end{array}$ & Participants & $\begin{array}{l}\text { Theoretical } \\
\text { background/ } \\
\text { Pedagogical } \\
\text { model }\end{array}$ & Intervention description & $\begin{array}{l}\text { Behavior change } \\
\text { techniques }\end{array}$ & $\begin{array}{l}\text { Behavior change } \\
\text { technique clusters }\end{array}$ & Motivational outcomes & Physical activity outcomes \\
\hline $\begin{array}{l}\text { Lonsdale et al. } \\
\text { (2019); } \\
\text { AustraliaRCT }\end{array}$ & $\begin{array}{l}\mathrm{N}=1421 \text { grade } 8 \\
\text { students. IG: } \mathrm{n}= \\
693(51.9 \% \text { boys), } \\
\text { age: } 12.96(0.56) \\
\text { years; CG: } \mathrm{n}=728 \text {, } \\
\text { age=12.9 }(0.52) \\
\text { years. }\end{array}$ & $\begin{array}{l}\text { based on self- } \\
\text { determination } \\
\text { theory tenets to } \\
\text { increase students' } \\
\text { satisfaction of } \\
\text { basic } \\
\text { psychological } \\
\text { needs }\end{array}$ & $\begin{array}{l}\text { keeping score, and one } \\
\text { 'social' game with no } \\
\text { recording of score. } \\
\text { Teachers were instructed } \\
\text { on implementation of } \\
\text { teaching strategies. CG: } \\
\text { teachers continued with } \\
\text { usual teaching practice. } \\
\text { IG: over one school year, } \\
\text { students participated in } \\
\text { "Activity and Motivation } \\
\text { in Physical Education" } \\
\text { classes. To maximize } \\
\text { MVPA, teachers' learnt } \\
\text { strategies in: (A) } \\
\text { 'Maximizing Movement } \\
\text { and Skill Development' } \\
\text { and (B) 'Reducing } \\
\text { Transition Time'. } \\
\text { Strategies to enhance } \\
\text { student motivation were } \\
\text { organized under: (C) } \\
\text { 'Building Competence' } \\
\text { and (D) 'Supporting } \\
\text { Students'. Teachers were } \\
\text { training through face-to- } \\
\text { face workshops, the } \\
\text { internet and a mobile app. } \\
\text { CG: students participated } \\
\text { in usual PE classes. }\end{array}$ & $\begin{array}{l}\text { 2.2. Feedback on } \\
\text { behavior3.1. Social support } \\
\text { (unspecified)4.1. } \\
\text { Instruction on how to } \\
\text { perform the behavior6.1. } \\
\text { Demonstration of the } \\
\text { behavior8.1. Behavioral } \\
\text { practice/rehearsal12.2. } \\
\text { Restructuring the social } \\
\text { environment }\end{array}$ & $\begin{array}{l}\text { 2. Feedback and } \\
\text { monitoring3. Social } \\
\text { support4. Shaping } \\
\text { knowledge6. } \\
\text { Comparison of } \\
\text { behavior8. Repetition } \\
\text { and substitution12. } \\
\text { Antecedents }\end{array}$ & $\begin{array}{l}\text { amotivation in } \mathrm{PE} / \text { leisure timeautonomous motivation in } \mathrm{PE} / \\
\text { leisure timecontrolled motivation in PE/ leisure } \\
\text { time**autonomy in PEcompetence in PErelatedness in } \\
\text { PEsupportive teacher behavior in PEcontrolled teacher } \\
\text { behavior in } \mathrm{PE}\end{array}$ & $\begin{array}{l}\text { in-class LPA (\%)** } \\
\text { (accelerometer)in-class } \\
\text { MPA (\%)**in-class VPA } \\
(\%)^{* *} \text { in-class MVPA (\%)** } \\
\text { in-class sedentary time (\%) } \\
* * \text { leisure time LPA (\%) } \\
\text { (accelerometer)leisure } \\
\text { time MPA (\%)**leisure } \\
\text { time MVPA (\%)**keisure } \\
\text { time VPA (\%)leisure time } \\
\text { sedentary time (\%)self- } \\
\text { reported leisure time PA } \\
\text { frequencyself-reported } \\
\text { leisure time PA duration }\end{array}$ \\
\hline $\begin{array}{l}\text { Méndez-Giménez } \\
\text { et al. (2015); } \\
\text { SpainCT }\end{array}$ & $\begin{array}{l}\mathrm{N}=295 \text { 7th to } 11 \text { th } \\
\text { grade students } \\
\text { (159 boys); age: } \\
14.2(1.68) . \mathrm{IG}_{1}: \\
\mathrm{n}=107 ; \mathrm{IG}_{2}: \mathrm{n}=78 ; \\
\text { CG: } \mathrm{n}=110 .\end{array}$ & $\begin{array}{l}\text { AGT, SDT/ Sport } \\
\text { Education Model }\end{array}$ & $\begin{array}{l}\mathrm{IG}_{1} \text { : over } 12 \text { sessions, } \\
\text { students participated in } \mathrm{PE} \\
\text { classes conducted } \\
\text { according to Sport } \\
\text { Education Model in an } \\
\text { Ultimate-Frisbee unit with } \\
\text { conventional resources, } 50 \\
\text { minutes each session. } \mathrm{IG}_{2} \text { : } \\
\text { students participated in PE } \\
\text { classes conducted in } \\
\text { accordance with the Sport } \\
\text { Education Model in an } \\
\text { Ultimate-Frisbee unit with } \\
\text { self-made flying rings. CG: } \\
\text { students participated in } \\
\text { traditional PE classes. }\end{array}$ & $\begin{array}{l}\text { 2.2. Feedback on } \\
\text { behavior6.2. Social } \\
\text { comparison10.10. Reward } \\
\text { (outcome) } 12.2 \text {. } \\
\text { Restructuring the social } \\
\text { environment }\end{array}$ & $\begin{array}{l}\text { 2. Feedback and } \\
\text { monitoring6. } \\
\text { Comparison of } \\
\text { behavior10. Reward } \\
\text { and threat12. } \\
\text { Antecedents }\end{array}$ & $\begin{array}{l}\text { mastery approach and mastery avoidance performance } \\
\text { approach and performance } \\
\text { avoidanceautonomy** competencerelatedness }\end{array}$ & $\mathrm{x}$ \\
\hline $\begin{array}{l}\text { Meng et al. (2013); } \\
\text { AustraliaCT }\end{array}$ & $\begin{array}{l}\mathrm{N}=257 \text { students } \\
\text { year } 8 \text { students, } \\
\text { age:12.91 }(0.29) \\
\text { years. IG: } \mathrm{n}=120 \text {, } \\
\text { Option } 1 \mathrm{n}=55 \text { ( } 35 \\
\text { boys), Option } 2\end{array}$ & SDT & $\begin{array}{l}\text { Over } 15 \text { weeks the groups } \\
\text { participated in } 3 \times 5 \text {-week- } \\
\text { units of netball, tennis, } \\
\text { and tee-ball. IG: students } \\
\text { had } 3 \text { options to choose } \\
\text { from 1) participating in }\end{array}$ & $\begin{array}{l}\text { 12.2. Restructuring the } \\
\text { social environment }\end{array}$ & 12. Antecedents & SDIteacher autonomy support & $\begin{array}{l}\text { boys in-class MVPA (\%) } \\
\text { (accelerometer)** girls in- } \\
\text { class MVPA** }\end{array}$ \\
\hline
\end{tabular}

keeping score, and one

recording of score.

Teachers were instructed

on

teachers continued with

usual teaching practice.

G: over one school year,

"Activity and Motivation

(B) 'Reducing

udent motivation were

'Building Competence'

(D) Supporing

raining through face-to-

ace workshops, the

téndez-Giménez $\mathrm{N}=2957$ th to 11 grade students

(159 boys); age:

classes conducted

$14.2(1.68)$ IG

$\mathrm{n}=107 ; \mathrm{IG}_{2}: \mathrm{n}=78$;

CG: $\mathrm{n}=110$.

boys), Option 2 


\begin{tabular}{|c|c|c|c|c|c|c|c|}
\hline $\begin{array}{l}\text { Author; } \\
\text { CountryDesign }\end{array}$ & Participants & $\begin{array}{l}\text { Theoretical } \\
\text { background/ } \\
\text { Pedagogical } \\
\text { model }\end{array}$ & Intervention description & $\begin{array}{l}\text { Behavior change } \\
\text { techniques }\end{array}$ & $\begin{array}{l}\text { Behavior change } \\
\text { technique clusters }\end{array}$ & Motivational outcomes & Physical activity outcomes \\
\hline & $\begin{array}{l}\mathrm{n}=31 \text { (11 boys), } \\
\text { Option } 3 \mathrm{n}=34 \text { (18 } \\
\text { boys); CG: } \mathrm{n}=137\end{array}$ & & $\begin{array}{l}\text { the unit as presented by PE } \\
\text { teacher, 2) acting as a "PE } \\
\text { development officer", or } \\
\text { 3) planning and } \\
\text { undertaking own personal } \\
\text { PA program. Option } 3 \\
\text { required: (a) minimum of } \\
30 \text { minutes of continuous } \\
\text { aerobic activity; (b) whole } \\
\text { body or large muscle } \\
\text { activities; (c) } \\
\text { predominantly moderate } \\
\text { to high intensity (using } \\
\text { perceived exertion } \\
\text { ratings); (d) activities } \\
\text { performed within view of } \\
\text { teacher. CG: students took } \\
\text { part in regular PE classes } \\
\text { with units of netball, } \\
\text { tennis and tee-ball. }\end{array}$ & & & & \\
\hline $\begin{array}{l}\text { Meng \& Keng } \\
\text { (2016); } \\
\text { SingaporeCT }\end{array}$ & $\begin{array}{l}\mathrm{N}=648 \text { secondary } \\
\text { school students } \\
\text { ( } 334 \text { boys), } \\
\text { age: } 14.35 \text { years; } \\
\mathrm{IG}_{1}: \mathrm{n}=173 ; \mathrm{IG}_{2} \text { : } \\
\mathrm{n}=239 ; \mathrm{CG}: \\
\mathrm{n}=236 .\end{array}$ & SDT & $\begin{array}{l}\mathrm{IG}_{1} \text { : over } 10 \text { weeks, } \\
\text { students participated in an } \\
\text { Autonomy-Supportive } \\
\text { Teaching Structure-PE } \\
\text { program during their PE } \\
\text { classes. } \mathrm{IG}_{2} \text { : students took } \\
\text { part in PE lessons with an } \\
\text { autonomy-supportive } \\
\text { motivational teaching } \\
\text { style only (AS). The } \\
\text { teachers of both } \\
\text { intervention groups } \\
\text { received training, training } \\
\text { hours depended on the } \\
\text { intervention. CG: students } \\
\text { participated in regular PE } \\
\text { classes. All lessons were } 60 \\
\text { minutes long. }\end{array}$ & $\begin{array}{l}\text { 1.1. Goal setting (behavior) } \\
\text { 2.2. Feedback on } \\
\text { behavior3.2. Social support } \\
\text { (practical) 4.1. Instruction } \\
\text { on how to perform the } \\
\text { behavior5.1. Information } \\
\text { about health } \\
\text { consequences6.1. } \\
\text { Demonstration of the } \\
\text { behavior8.1. Behavioral } \\
\text { practice/ rehearsal }\end{array}$ & $\begin{array}{l}\text { 1. Goals and planning2. } \\
\text { Feedback and } \\
\text { monitoring3. Social } \\
\text { support4. Shaping } \\
\text { knowledge5. Natural } \\
\text { consequences6. } \\
\text { Comparison of } \\
\text { behavior8. Repetition } \\
\text { and substitution }\end{array}$ & $\begin{array}{l}\text { teacher autonomy } \\
\text { support**autonomy }{ }^{* *} \text { competence }^{* *} \text { relatedness*RAI** }\end{array}$ & $\begin{array}{l}\text { overall in-class MVPA \% } \\
\text { (accelerometer)* boys in- } \\
\text { class MVPA\%** girls in- } \\
\text { class MVPA\%* }\end{array}$ \\
\hline $\begin{array}{l}\text { Moreno et al. } \\
\text { (2010);SpainCT }\end{array}$ & $\begin{array}{l}\mathrm{N}=363 \text { students } \\
\text { (227 boys), age: } \\
13.21 \text { (0.9) years. } \\
\mathrm{IG}_{1}: \mathrm{n}=121 ; \mathrm{IG}_{2}: \\
\mathrm{n}=121 ; \mathrm{CG}: \\
\mathrm{n}=12.1\end{array}$ & SDT & $\begin{array}{l}\text { During one session, two } \\
\text { groups were manipulated } \\
\text { regarding their ability } \\
\text { beliefs during a motor } \\
\text { task. Both groups received } \\
\text { different information } \\
\text { using football players as } \\
\text { models. } \mathrm{IG}_{1} \text { : experimental- } \\
\text { incremental group; } \\
\text { manipulation based on the } \\
\text { fact that sport abilities can } \\
\text { be developed through } \\
\text { learning, practice and } \\
\text { training. } \mathrm{IG}_{2}:\end{array}$ & $\begin{array}{l}\text { 4.1. Instruction on how to } \\
\text { perform the behavior13.2. } \\
\text { Framing/reframing }\end{array}$ & $\begin{array}{l}\text { 4. Shaping knowledge } \\
\text { 13. Identity }\end{array}$ & task competencesituational intrinsic motivation** & $\mathrm{x}$ \\
\hline
\end{tabular}

the unit as presented by $P$ teacher, 2) acting as a "PE

3) planning and

undertaking own person

A program. Option 3

30 minutes of continuous

aerobic activity; (b) whol

redominantly moderate

herceived exertion

ratings); (d) activitie

performed within view of

1. Goal setting (behavior) 1. Goals and planning2 teacher autonomy

school students

age:14.35 yea

$\mathrm{IG}_{1}: \mathrm{n}=173 ; \mathrm{IG}_{2}$ :

$\mathrm{n}=239$; CC:

$\mathrm{N}=363$ students

(227 boys), age:

$\mathrm{IG}_{1}: \mathrm{n}=121 ; \mathrm{IG}_{2}$ :

$\mathrm{n}=121$; $\mathrm{CG}$

$\mathrm{n}=12.1$. 


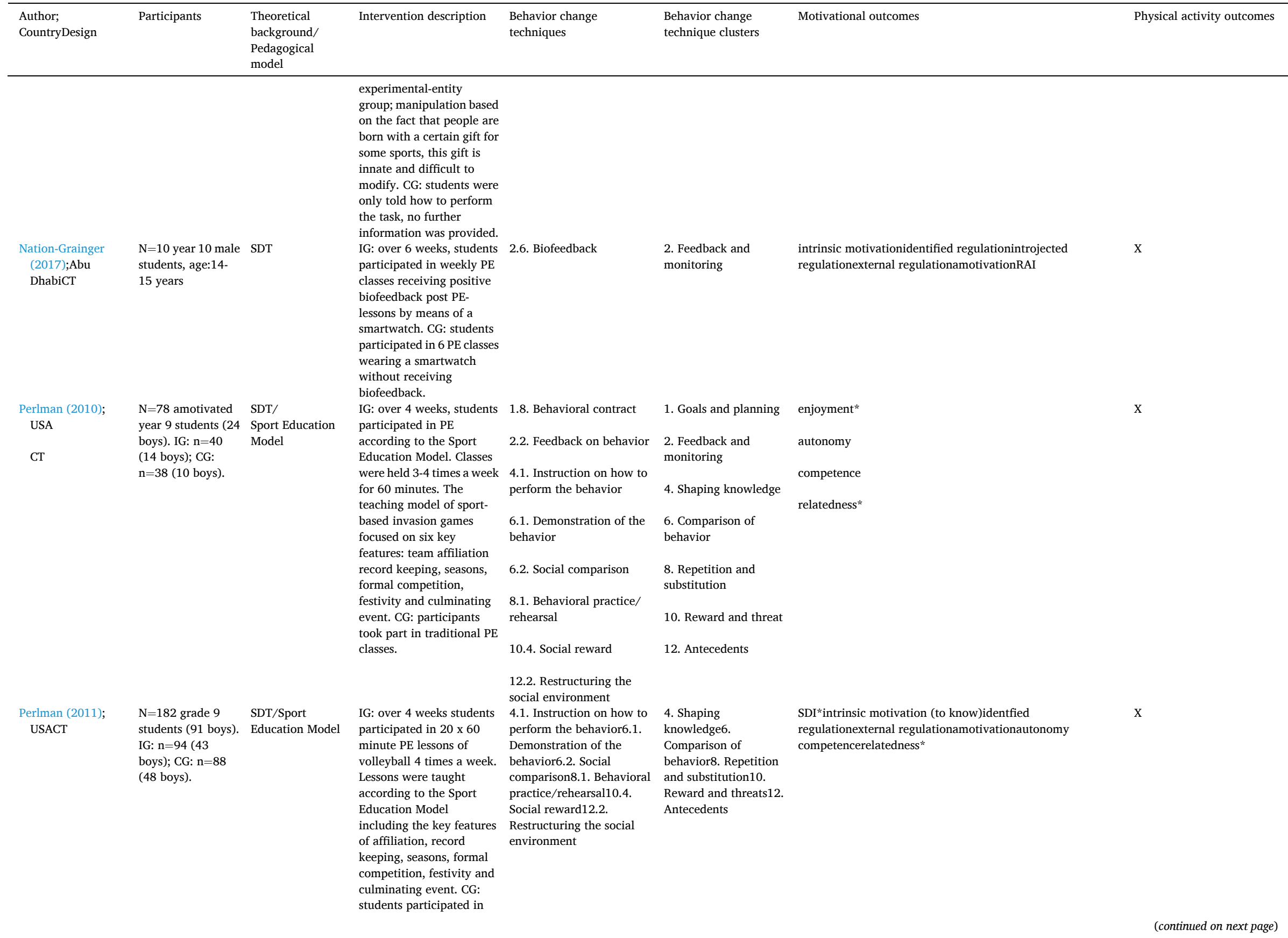

experimental-entity

group, manipulation based

ome sports, this gift is

modify. CG: students were

only told how to perfor

lasses receiving positive

ofeedback post $\mathrm{PE}$ -

martwatch. CG: studen

wearing a smartwatch

wint

G: over 4 weeks,

articipated in $\mathrm{PE}$

for 60 minutes. The

focused on six key

ormal competition,

estivity and culminating

event. CG: participants

took part
classes.

10.4. Social reward

. Reward and threat 


\begin{tabular}{|c|c|c|c|c|c|c|c|}
\hline $\begin{array}{l}\text { Author; } \\
\text { CountryDesign }\end{array}$ & Participants & $\begin{array}{l}\text { Theoretical } \\
\text { background/ } \\
\text { Pedagogical } \\
\text { model }\end{array}$ & Intervention description & $\begin{array}{l}\text { Behavior change } \\
\text { techniques }\end{array}$ & $\begin{array}{l}\text { Behavior change } \\
\text { technique clusters }\end{array}$ & Motivational outcomes & $\mathrm{Ph}$ \\
\hline & & & $\begin{array}{l}\text { traditional lessons of } \\
\text { volleyball. }\end{array}$ & & & & \\
\hline $\begin{array}{l}\text { Perlman (2015); } \\
\quad \text { USACT }\end{array}$ & $\begin{array}{l}\mathrm{N}=48 \text { amotivated } \\
\text { high-school } \\
\text { students ( } 18 \text { boys). } \\
\text { IG: } \mathrm{n}=24 \text { ( } 9 \text { boys); } \\
\text { CG: } \mathrm{n}=24 \text { ( } 9 \text { boys). }\end{array}$ & SDT & $\begin{array}{l}\text { IG: students participated } \\
\text { in } 16 \text { PE lessons, } \\
\text { approximately } 4 \text { weeks, of } \\
\text { basketball with a high } \\
\text { level of autonomy support. } \\
\text { The teacher received an } \\
\text { autonomy-supportive } \\
\text { workshop. CG: students } \\
\text { took part in } 16 \text { PE classes } \\
\text { of basketball with a } \\
\text { consistent level of } \\
\text { autonomy-supportive and } \\
\text { controlling instruction. }\end{array}$ & $\begin{array}{l}\text { 4.1. Instruction on how to } \\
\text { perform the behavior6.1. } \\
\text { Demonstration of the } \\
\text { behavior8.1. Behavioral } \\
\text { practice/rehearsal }\end{array}$ & $\begin{array}{l}\text { 4. Shaping } \\
\text { knowledge6. } \\
\text { Comparison of } \\
\text { behavior8. Repetition } \\
\text { and substitution }\end{array}$ & $\begin{array}{l}\text { teacher autonomy-support**} \\
\text { SDI I**autonomycompetencerelatedness** }^{* *}\end{array}$ & $\mathrm{x}$ \\
\hline $\begin{array}{l}\text { Prusak et al. (2004); } \\
\text { USACT }\end{array}$ & $\begin{array}{l}\mathrm{N}=1110 \text { 7th and } \\
\text { 8th grade female } \\
\text { students from } 42 \\
\text { classes. IG: } \mathrm{n}=21 \\
\text { classes; CG: } \mathrm{n}=21 \\
\text { classes. }\end{array}$ & $\begin{array}{l}\text { SDT, Hierarchical } \\
\text { model of } \\
\text { motivation }\end{array}$ & $\begin{array}{l}\text { IG: over } 10 \text { days, students } \\
\text { participated in walking } \\
\text { activities, } 50 \text { minutes each } \\
\text { day during their regular } \\
\text { PE classes. Students were } \\
\text { allowed to make choices } \\
\text { during the units on which } \\
\text { activities they would be } \\
\text { participating in and with } \\
\text { whom. The teachers of the } \\
\text { CG and IG received a } \\
\text { workshop on instructional } \\
\text { and experimental } \\
\text { procedures specific for } \\
\text { each treatment group. CG: } \\
\text { students took part in } \\
\text { walking activities during } \\
\text { their regular PE classes, } \\
\text { without having a choice as } \\
\text { to which activity they } \\
\text { would participate in and } \\
\text { with whom. }\end{array}$ & $\begin{array}{l}\text { 4.1. Instruction on how to } \\
\text { perform the behavior5.1. } \\
\text { Information about health } \\
\text { consequences6.1. } \\
\text { Demonstration of the } \\
\text { behavior8.1. Behavioral } \\
\text { practice12.2. Restructuring } \\
\text { the social environment }\end{array}$ & $\begin{array}{l}\text { 4. Shaping } \\
\text { knowledge5. Natural } \\
\text { consequences6. } \\
\text { Comparison of } \\
\text { behavior8. Repetition } \\
\text { and substitution12. } \\
\text { Antecedents }\end{array}$ & $\begin{array}{l}\text { contextual self-determination (including intrinsic motivation, } \\
\text { extrinsic motivationamotivation) }\end{array}$ & $\mathrm{x}$ \\
\hline $\begin{array}{l}\text { Sevil et al. (2016); } \\
\text { SpainCT }\end{array}$ & $\begin{array}{l}\mathrm{N}=224 \text { secondary } \\
\text { school students } \\
\text { (105 boys), age: } \\
12.37 \text { ( } 0.64 \text { ) years. } \\
\text { IG: } \mathrm{n}=109 \text { ( } 51 \\
\text { boys); CG: } \mathrm{n}=115 \\
\text { ( } 54 \text { boys). }\end{array}$ & $\begin{array}{l}\text { SDT, AGT/ } \\
\text { Tactical games } \\
\text { model (TGM), } \\
\text { TARGET }\end{array}$ & $\begin{array}{l}\text { IG: students participated } \\
\text { in } 10 \text { sessions of rope- } \\
\text { skipping activities in PE } \\
\text { geared towards corporal } \\
\text { expression, twice a week } \\
\text { for } 50 \text { minutes each. } \\
\text { Lessons were conducted in } \\
\text { accordance with TARGET } \\
\text { strategies. The teacher } \\
\text { took part in a 5-week } \\
\text { training program prior to } \\
\text { the intervention. CG: } \\
\text { students took part in } 10 \\
\text { sessions of rope skipping } \\
\text { activities. TGM was used }\end{array}$ & $\begin{array}{l}\text { 2.4. Self-monitoring of } \\
\text { outcome(s) of behavior } 2.7 \text {. } \\
\text { Feedback on outcome(s) of } \\
\text { behavior4.1. Instruction on } \\
\text { how to perform the } \\
\text { behavior6.1. } \\
\text { Demonstration of the } \\
\text { behavior8.1. Behavioral } \\
\text { practice/rehearsal12.2. } \\
\text { Restructuring the social } \\
\text { environment }\end{array}$ & $\begin{array}{l}\text { 2. Feedback and } \\
\text { monitoring } \\
\text { 4. Shaping knowledge } \\
\text { 6. Comparison of } \\
\text { behaviour } \\
\text { 8. Repetition and } \\
\text { substitution } \\
\text { 12. Antecedents }\end{array}$ & $\begin{array}{l}\text { task-oriented climate**ego-oriented } \\
\text { climate**autonomy*competence }{ }^{* *} \text { relatednessintrinsic } \\
\text { motivation } * * \text { identified regulation**enjoyment/ } \\
\text { satsifaction }{ }^{* *} \text { extrinsic regulationamotivation }\end{array}$ & $\mathrm{x}$ \\
\hline
\end{tabular}




\begin{tabular}{|c|c|c|c|c|c|c|c|}
\hline $\begin{array}{l}\text { Author; } \\
\text { CountryDesign }\end{array}$ & Participants & $\begin{array}{l}\text { Theoretical } \\
\text { background/ } \\
\text { Pedagogical } \\
\text { model }\end{array}$ & Intervention description & $\begin{array}{l}\text { Behavior change } \\
\text { techniques }\end{array}$ & $\begin{array}{l}\text { Behavior change } \\
\text { technique clusters }\end{array}$ & Motivational outcomes & Phy \\
\hline & & & $\begin{array}{l}\text { as the pedagogical model } \\
\text { in both groups. }\end{array}$ & & & & \\
\hline $\begin{array}{l}\text { Smith et al. (2018); } \\
\text { AustraliaRCT }\end{array}$ & $\begin{array}{l}\mathrm{N}=607 \text { grade } 9 \\
\text { students ( } 50.4 \% \\
\text { boys), age: } 14.1 \\
(0.5) \text { years. IG: } \\
\mathrm{n}=353, \text { age: } 14.1 \\
(0.4) \text { years; CG: } \\
\mathrm{n}=254, \text { age: } 14.2 \\
(0.5) \text { years. }\end{array}$ & $\begin{array}{l}\text { SDT/SAAFE } \\
\text { teaching } \\
\text { principles }\end{array}$ & $\begin{array}{l}\text { IG: over } 10 \text { weeks, } \\
\text { students participated in } \\
\text { resistance training and } \\
\text { other health and fitness } \\
\text { activities. Intervention } \\
\text { components were: an } \\
\text { introductory seminar for } \\
\text { students, weekly PA } \\
\text { sessions (about } 90 \\
\text { minutes) during PE, PA, } \\
\text { and Sport Studies elective } \\
\text { class, lunch-time activity } \\
\text { sessions ( } 5 \text { x } 20 \text { min), and } \\
\text { a web-based smartphone } \\
\text { application to supplement } \\
\text { face-to-face components. } \\
\text { Teachers received a } \\
\text { workshop to deliver } \\
\text { intervention program. CG: } \\
\text { students and teachers } \\
\text { received intervention after } \\
\text { the study period. }\end{array}$ & $\begin{array}{l}\text { 1.1. Goal setting (behavior) } \\
\text { 1.5. Review behavior } \\
\text { (goals)2.3. Self-monitoring } \\
\text { of behavior 4.1. Instruction } \\
\text { on how to perform the } \\
\text { behavior5.1. Information } \\
\text { about health } \\
\text { consequences6.1. } \\
\text { Demonstration of the } \\
\text { behavior8.1. Behavioral } \\
\text { practice/rehearsal12.2. } \\
\text { Restructuring the social } \\
\text { environment12.5. Adding } \\
\text { objects to the environment }\end{array}$ & $\begin{array}{l}\text { 1. Goals and planning2. } \\
\text { Feedback and } \\
\text { monitoring4. Shaping } \\
\text { knowledge5. Natural } \\
\text { consequences6. } \\
\text { Comparison of } \\
\text { behavior8. Repetition } \\
\text { and substitution12. } \\
\text { Antecedents }\end{array}$ & intrinsic motivation identified regulation & $\mathrm{x}$ \\
\hline $\begin{array}{l}\text { Spittle \& Byrne } \\
\text { (2009); } \\
\text { AustraliaCT }\end{array}$ & $\begin{array}{l}\mathrm{n}=115 \text { year } 8 \\
\text { students ( } 97 \text { boys), } \\
\text { age }=13-14 \text { years. } \\
\text { IG: } \mathrm{n}=41 \text { ( } 32 \\
\text { boys); CG: } \mathrm{n}=74 \\
\text { ( } 65 \text { boys). }\end{array}$ & $\begin{array}{l}\text { AGT/Sport } \\
\text { Education Model }\end{array}$ & $\begin{array}{l}\text { IG: over } 10 \text { weeks, } \\
\text { students in the Sport } \\
\text { Education condition took } \\
\text { part in one double period } \\
\text { of PE (100 minutes) once a } \\
\text { week. The intervention } \\
\text { incorporated } 6 \text { features: } \\
\text { seasons, affiliation, formal } \\
\text { competition, record } \\
\text { keeping, festivity, and a } \\
\text { culminating event. It } \\
\text { followed a 3-phase format } \\
\text { 1) initial, teacher-directed, } \\
\text { skill development phase; } \\
\text { 2) student-led activities } \\
\text { and games phase; } 3 \text { ) } \\
\text { formal competition phase. } \\
\text { CG: participants took part } \\
\text { in traditional PE classes. }\end{array}$ & $\begin{array}{l}\text { 4.1. Instruction on how to } \\
\text { perform the behavior6.1. } \\
\text { Demonstration of the } \\
\text { behavior6.2. Social } \\
\text { comparison8.1. Behavioral } \\
\text { practice/rehearsal10.4. } \\
\text { Social reward12.2. } \\
\text { Restructuring the social } \\
\text { environment }\end{array}$ & $\begin{array}{l}\text { 4. Shaping } \\
\text { knowledge6. } \\
\text { Comparison of } \\
\text { behavior8. Repetition } \\
\text { and substitution10. } \\
\text { Reward and threat12. } \\
\text { Antecedents }\end{array}$ & $\begin{array}{l}\text { competence } * \text { interest/enjoymenttask goal orientation }{ }^{* *} \text { ego } \\
\text { goal orientation mastery climate }{ }^{* *} \text { performance climate }\end{array}$ & $\mathrm{x}$ \\
\hline $\begin{array}{l}\text { Wallhead } \\
\text { \&Ntoumanis } \\
\text { (2004); } \\
\text { EnglandCT }\end{array}$ & $\begin{array}{l}\mathrm{N}=51 \text { male high } \\
\text { school students, } \\
\text { age: } 14.3(0.48) \\
\text { years. IG: } \mathrm{n}=26 \text {; } \\
\text { CG: } \mathrm{n}=25 \text {. }\end{array}$ & $\begin{array}{l}\text { AGT/Sport } \\
\text { Education Model }\end{array}$ & $\begin{array}{l}\text { IG: over } 8 \text { weeks, students } \\
\text { participated in weekly 1- } \\
\text { hour PE classes following } \\
\text { the Sport Education } \\
\text { Model. The teaching } \\
\text { approach followed a three- } \\
\text { phase format: a teacher- } \\
\text { directed skill development } \\
\text { phase, a preseason }\end{array}$ & $\begin{array}{l}\text { 1.8. Behavioral } \\
\text { contract4.1. Instruction on } \\
\text { how to perform the } \\
\text { behavior6.1. } \\
\text { Demonstration of the } \\
\text { behavior } 6.2 \text {. Social } \\
\text { comparison8.1. Behavioral } \\
\text { practice/rehearsal12.2. }\end{array}$ & $\begin{array}{l}\text { 1. Goals and planning } 4 \text {. } \\
\text { Shaping knowledge6. } \\
\text { Comparison of } \\
\text { behavior8. Repetition } \\
\text { and substitution12. } \\
\text { Antecedents }\end{array}$ & $\begin{array}{l}\text { enjoyment**autonomycompetence task goal orientationego } \\
\text { goal orientationtask climateperformance climate }\end{array}$ & $\mathrm{x}$ \\
\hline
\end{tabular}




\begin{tabular}{|c|c|c|c|c|c|c|c|}
\hline $\begin{array}{l}\text { Author; } \\
\text { CountryDesign }\end{array}$ & Participants & $\begin{array}{l}\text { Theoretical } \\
\text { background/ } \\
\text { Pedagogical } \\
\text { model }\end{array}$ & Intervention description & $\begin{array}{l}\text { Behavior change } \\
\text { techniques }\end{array}$ & $\begin{array}{l}\text { Behavior change } \\
\text { technique clusters }\end{array}$ & Motivational outcomes & Physical activity outcomes \\
\hline & & & $\begin{array}{l}\text { scrimmage phase, a formal } \\
\text { competition phase. During } \\
\text { each phase students acted } \\
\text { in the roles of being a } \\
\text { coach, referee, captain, } \\
\text { and scorer. Students on } \\
\text { each team selected } \\
\text { individuals to fulfill each } \\
\text { role. CG: students } \\
\text { participated in traditional } \\
\text { PE. }\end{array}$ & $\begin{array}{l}\text { Restructuring the social } \\
\text { environment }\end{array}$ & & & \\
\hline $\begin{array}{l}\text { Wallhead et al. } \\
\text { (2014);USACT }\end{array}$ & $\begin{array}{l}\mathrm{N}=538 \text { high school } \\
\text { students, age: } 14.75 \\
\text { (0.48) years. IG: } \\
\mathrm{n}=261 \text {; CG: } \\
\mathrm{n}=277 .\end{array}$ & $\begin{array}{l}\text { SDT/Sport } \\
\text { Education Model }\end{array}$ & $\begin{array}{l}\text { IG: over two years, } \\
\text { students participated in } 4 \\
\text { seasons (each } 25 \text { lessons) } \\
\text { of team sport activities in } \\
\text { accordance with the Sport } \\
\text { Education Program. } \\
\text { Lessons were held } 90 \\
\text { minutes, } 2-3 \text { times per } \\
\text { week. Following the initial } \\
\text { management phase, each } \\
\text { season followed a protocol } \\
\text { of teacher-directed skill } \\
\text { development, a preseason } \\
\text { coach-directed phase, a } \\
\text { formal tournament, and } \\
\text { postseason culminating } \\
\text { event. Student role } \\
\text { responsibilities included } \\
\text { coach, referee and scorer. } \\
\text { CG: students participated } \\
\text { in a multi-activity } \\
\text { program during their PE } \\
\text { lessons. Instructions were } \\
\text { provided by the teacher; } \\
\text { feedback during game play } \\
\text { was limited, students were } \\
\text { not accountable for game } \\
\text { performance. }\end{array}$ & $\begin{array}{l}\text { 4.1. Instruction on how to } \\
\text { perform the behavior6.1. } \\
\text { Demonstration of the } \\
\text { behavior6.2. Social } \\
\text { comparison8.1. Behavioral } \\
\text { practice/rehearsal12.2. } \\
\text { Restructuring the social } \\
\text { environment }\end{array}$ & $\begin{array}{l}\text { 4. Shaping } \\
\text { knowledge6. } \\
\text { Comparison of } \\
\text { behavior8. Repetition } \\
\text { and substitution12. } \\
\text { Antecedents }\end{array}$ & enjoyment** & $\begin{array}{l}\text { self-reported leisure time } \\
\mathrm{PA}^{*}\end{array}$ \\
\hline $\begin{array}{l}\text { Weigand \& Burton } \\
\text { (2002);EnglandCT }\end{array}$ & $\begin{array}{l}\mathrm{N}=40 \text { students ( } 32 \\
\text { boys), age: } 15.9 \\
\text { (0.51) years. IG: } \\
\mathrm{n}=20 ; \text { CG: } \mathrm{n}=20 \text {. }\end{array}$ & AGT/TARGET & $\begin{array}{l}\text { IG: over } 5 \text { weeks, students } \\
\text { participated in 1-hour PE } \\
\text { classes promoting a } \\
\text { mastery climate using the } \\
\text { TARGET approach. CG: } \\
\text { students participated in } \\
\text { regular PE classes with } \\
\text { same lesson content. }\end{array}$ & $\begin{array}{l}\text { 1.1. Goal setting (behavior) } \\
\text { 2.7. Feedback on outcome } \\
\text { (s) of behavior } 4.1 \\
\text { Instruction on how to } \\
\text { perform the behavior } 6.1 \\
\text { Demonstration of the } \\
\text { behavior8.1. Behavioral } \\
\text { practice/rehearsal12.2. } \\
\text { Restructuring the social } \\
\text { environment }\end{array}$ & $\begin{array}{l}\text { 1. Goals and planning2. } \\
\text { Feedback and } \\
\text { monitoring4. Shaping } \\
\text { knowledge5. Natural } \\
\text { consequences } 6 . \\
\text { Comparison of } \\
\text { behavior8. Repetition } \\
\text { and substitution12. } \\
\text { Antecedents }\end{array}$ & $\begin{array}{l}\text { task goal orientation** ego goal orientation** } \\
\text { competence**perceived satisfaction/enjoyment }{ }^{* *}\end{array}$ & $\mathrm{x}$ \\
\hline $\begin{array}{l}\text { Yli-Piipari et al. } \\
\text { (2018); USARCT }\end{array}$ & $\begin{array}{l}\mathrm{N}=408 \text { middle } \\
\text { school students } \\
\text { (192 boys), }\end{array}$ & $\begin{array}{l}\text { Trans-contextual } \\
\text { model of } \\
\text { motivation }\end{array}$ & $\begin{array}{l}\text { IG: over } 8 \text { weeks, students } \\
\text { participated in autonomy } \\
\text { supportive PE classes. The }\end{array}$ & No information provided & $\begin{array}{l}\text { No information } \\
\text { provided }\end{array}$ & During week 4:autonomy supportive climate**RAI (PE)*** & self-reported PA** \\
\hline
\end{tabular}

scrimmage phase, a formal Restructuring the social

comption phase. During environment

in the ros of being a

coach, referee, captain,

a

individuals to fulfill eac

role. CG: students

participated in tradition

IG: over two years,

4.1. Instruction on how to 4. Shaping

knowledge6.

of team sport activities in behavior6.2. Social behavior8. Repetition

accordance with the Sport comparison8.1. Behavioral and substitution12.

Education Program. $\quad$ practice/rehearsal12.2.

Restructuring the socia

ason followed a protoco

development, a preseaso

coach-directed phase, a

postseason culminating

coach, referee and scorer.

during their PE

provided by the teacher

eedback during game play

limited, students were

rformance.

.7. Feedback on outcome Feedback and

nstruction on how to knowledge5. Natura

estructuring the so

participated in autonomy

supportive PE classes. The 
the clusters 'Shaping knowledge', 'Comparison of behavior' and 'Antecedents' (all $\mathrm{n}=10$ ) followed by the clusters 'Repetition and substitution' ( $\mathrm{n}=9$ ), 'Feedback and monitoring' $(\mathrm{n}=9)$ and 'Goals and planning' $(\mathrm{n}=7)$.

\subsection{Primary outcomes}

The most frequently evaluated motivational outcomes were perceived competence $(n=30)$, enjoyment and satisfaction of PE or PA $(\mathrm{n}=26)$, students' perceived motivational climate in PE $(\mathrm{n}=19)$, perceived autonomy $(\mathrm{n}=18)$, intrinsic motivation $(\mathrm{n}=18)$, and perceived relatedness $(n=16)$. A few studies $(n=4)$ determined the level of autonomous motivation by measuring the relative autonomy index (RAI), calculated as: (external regulation * $(-2)+$ introjected regulation* $(-1)+$ identified regulation * $(+1)+$ intrinsic motivation * $(+2))$. A higher score represents a higher level of intrinsic motivation (Chatzisarantis and Hagger, 2009). Another index, frequently referred to, was the self-determination index (SDI) $(n=6)$ to measure the level of self-determination. Lonsdale et al., 2013 calculated this similarly to the RAI: $(2 *$ intrinsic motivation + identified motivation - external regulation $-2 *$ amotivation). Cuevas et al., 2016 determined global self-determination by calculating the SDI as: $(2 *$ intrinsic motivation $)+$ identified regulation - (introjected regulation + external regulation)/2-2* amotivation). Tables 1 and 2 show the outcomes of interest measured in the IG and CG at a minimum of two time-points (pre to post intervention). Significant results at post-test are indicated where applicable.

\subsection{Secondary outcomes}

Physical activity behavior was measured in 30 studies during PE lessons $(\mathrm{n}=13)$, during the school day $(\mathrm{n}=3)$, and in leisure time by means of questionnaires $(n=12)$, observations $(n=4)$, and measurement tools such as accelerometers and pedometers $(n=16)$. Of these studies, 28 conducted pre-post analyses of PA between groups. Tables 1 and 2 show the PA outcomes measured in these studies in IG and CG at a minimum of two time-points (pre to post intervention). Significant results at post-test are indicated where applicable.

\subsection{Methodological quality}

An overview of risk of bias for all included studies and each category is given in Fig. 2. Additional file 2 shows the risk of bias for each individual study. The study with the lowest risk of bias, was the RCT by Salmon et al. (2008). None of the RCTs had a low risk of bias in all eight categories, a minimum of two domains were rated with a high risk of bias in each examined study. Nearly all of the studies had a high risk of bias in the domains performance bias, i.e. blinding of participants and personnel, and detection bias, i.e. blinding of outcome assessment. The lowest risk of bias across studies was found in the domains reporting bias and other risk of bias, i.e. timing of measurement, the latter is not included in the standard Cochrane Collaboration's tool for assessing risk of bias in randomized trials.

\subsection{Intervention effects primary outcomes}

Overall, meta-analyses of 16 outcomes were performed with the number of included comparisons $(k)$ ranging between 4 and 28. Table 3 shows an overview of all calculated meta-analyses including the effect size statistic, the heterogeneity statistic and analysis of publication bias.

Enjoyment. The overall analysis of enjoyment resulted in a significant and small to moderate effect size $(g=0.310,95 \% \mathrm{CI}=0.080$ to $0.541, p$ $=0.008)$ with high heterogeneity $\left(I^{2}=87.730 \%\right)$. The effect size in CTs was small to moderate ( $g=0.404,95 \% \mathrm{CI}=0.148$ to $0.660, p=0.02$ ) and heterogeneity was high $\left(I^{2}=88.896 \%\right)$ A non-significant, negative effect size was found in RCTs $(p=0.113)$ with low heterogeneity $\left(I^{2}=\right.$
$15.979 \%)$.

Basic psychological needs. The analyses of perceived competence and relatedness resulted in non-significant, trivial effect sizes and high heterogeneity $\left(I^{2}=96.530 \%\right.$ and $I^{2}=75.727 \%$, respectively). The overall analyses of perceived autonomy resulted in a significant and small effect size ( $g=0.152,95 \% \mathrm{CI}=0.013$ to $0.290, p=0.032)$ and moderate to high $I^{2}(69.328 \%)$. A significant and small effect size was further found in CTs ( $g=0.196,95 \% \mathrm{CI}=0.020$ to $0.371, p=0.029$ ), whereas nonsignificant results were found in RCTs ( $\left.p=0.827, I^{2}=0.000 \%\right)$.

Motivation. The overall analyses of amotivation, external regulation, introjected regulation and RAI resulted in non-significant effect sizes. The analysis of identified regulation showed a small to moderate significant effect ( $g=0.378,95 \% \mathrm{CI}=0.041$ to $0.714, p=0.028$ ). A small to moderate significant effect size was also found for intrinsic motivation $(g=0.419,95 \% \mathrm{CI}=0.085$ to $0.752, p=0.014)$. An overall moderate to large significant effect size was found for SDI $(g=0.672,95 \% \mathrm{CI}=0.112$ to $1.232, p=0.019$ ) with a large and significant effect size in the CTs ( $g$ $=1.161,95 \% \mathrm{CI}=0.321$ to $2.001, p=0.007$ ). Overall heterogeneity was high in all variables, except for introjected regulation $\left(I^{2}=\right.$ $28.488 \%)$ and moderate to high for RAI $\left(I^{2}=69.138 \%\right)$

Motivational climate. The analysis of these outcomes showed significant effects. The highest effect size was found for ego/performance oriented teaching climate $(g=-0.438,95 \% \mathrm{CI}=-0.646$ to $-0.230, p<$ 0.01 ), meaning students in the IG had significantly lower values in their perception of ego/performance climate at post-test than the CG. The lowest effect size was found for task/mastery oriented teaching climate ( $g=0.254,95 \% \mathrm{CI}=0.097$ to $0.411, p=0.001$ ), meaning students in the IG had significantly larger scores in their perception of a mastery/ task climate at post-test than the CG. The heterogeneity was moderate $\left(I^{2}=60.509 \%\right.$ (task/mastery climate) to high $I^{2}=87.195 \%$ (autonomy supportive climate). A small and significant effect size was found in RCTs for autonomy supportive climate $(g=0.340,95 \% \mathrm{CI}=0.053$ to $0.627, p=0.020$ ), results in CTs were non-significant ( $p=0.436$ ).

Goal Orientation. A large and significant effect size was found for task goal orientation ( $g=1.370,95 \%=\mathrm{CI} 0.694$ to $2.045, p<0.01$ ) with high heterogeneity $\left(I^{2}=92.718 \%\right)$. The analysis of ego/performance goal orientation resulted in a small, significant effect size $(g=-0.188$, $95 \% \mathrm{CI}=-0.327$ to $-0.050, p=0.008)$ with low heterogeneity $\left(I^{2}=\right.$ $4.438 \%)$.

\subsection{Publication bias}

The inspection of the Funnel Plot and the Eggers' Test indicated no publication bias for most of the variables. The Eggers' Test was significant for introjected regulation ( $p=0.039$, two-tailed) and task goal orientation ( $p=0.024$, two-tailed) and indicated an asymmetric funnel plot.

\subsection{Subgroup analyses}

A series of pre-specified subgroup-moderator analyses were conducted. A summary of the calculated effect sizes and the heterogeneity statistic is presented in Table 4.

Studies examining enjoyment that were theory-based showed a significant and moderate effect size $(g=0.465,95 \% \mathrm{CI}=0.199$ to $0.732, p$ $=0.001$ ) while the effect size for studies that were not theory-based was trivial and non-significant $(g=-0.064,95 \% \mathrm{CI}=-0.475$ to $0.347, p=$ 0.759). A moderate, significant effect size was found in adolescents $(g=$ $0.471,95 \% \mathrm{CI}=0.147$ to $0.796, p=0.004)$ but not in children $(g=$ $0.114,95 \% \mathrm{CI}=-0.249$ to $0.478, p=0.537)$. A moderate and significant effect size was found in CTs $(g=0.402,95 \% \mathrm{CI}=0.153$ to $0.651, p$ $=0.002)$ but not in RCTs $(g=-0.157,95 \% \mathrm{CI}=-0.716$ to $0.402, p=$ 0.582). $Q$-statistics revealed significant differences only between the moderators theory-based and non-theory based studies $(p<0.05)$ but not between age groups or the different study designs $(p>0.05)$.

Basic psychological needs. Excluding outliers and comparisons with 


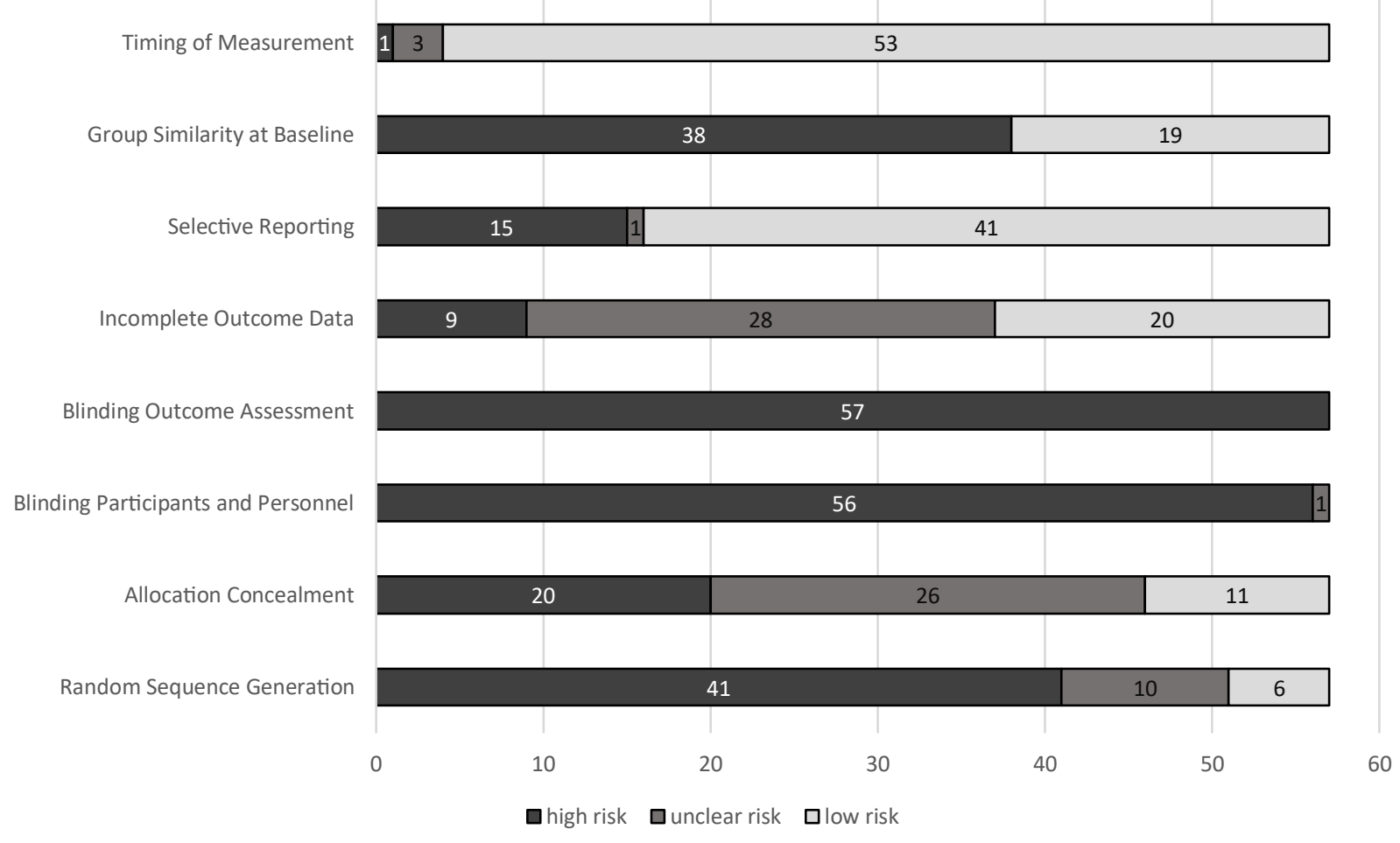

Fig. 2. Risk of bias across all included studies.

the highest and lowest effect sizes resulted in a reduction of heterogeneity. For perceived autonomy the effect size was larger and significant for children ( $g=0.407,95 \% \mathrm{CI}=0.144$ to $0.671, p=0.002$ ) compared to adolescents ( $g=0.074,95 \% \mathrm{CI}=-0.070$ to $0.219, p=0.314$ ) and for CTs $(g=0.196,95 \% \mathrm{CI}=0.037$ to $0.355, p=0.016)$ compared to RCTs $(g=0.001,95 \% \mathrm{CI}=-0.291$ to $0.294, p=0.934)$. Heterogeneity was further explained in children and in RCTs for perceived autonomy. $Q$ statistics showed significant differences only between the moderators children and adolescents $(p<0.05)$.

Motivation. The exclusion of the highest and lowest effect sizes ( $g=$ $0.404,95 \%=$ CI -0.062 to $0.869, p=0.089$ ) clearly decreased the effect size for SDI and resulted in non-significant effect estimates. Decreased effect sizes were also found for amotivation, external regulation, identified regulation, intrinsic motivation, and RAI. For intrinsic motivation the subgroup analyses revealed a small effect size in children $(g=0.290$, $95 \% \mathrm{CI}=-0.104$ to $0.683, p=0.149)$ and a moderate effect size for adolescents ( $\mathrm{g}=0.557,95 \% \mathrm{CI}=-0.057$ to $1.212, p=0.074$ ). Both results were non-significant and heterogeneity was high. $Q$-statistics showed significant differences between the moderators CT and RCT for identified regulation $(p<0.05)$, intrinsic motivation $(p<0.05)$, SDI $(p<$ $0.01)$, and RAI $(p<0.01)$.

Motivational climate. The exclusion of outliers and comparisons with the highest and lowest effect size decreased heterogeneity for all three variables. The effect size of the variable autonomy supportive climate increased with the exclusion of outliers and highest/lowest effect sizes, while the effect size of ego/performance climate slightly decreased. The effect estimates remained significant. $Q$-statistics showed significant differences between the moderators children and adolescents $(p<0.01)$ for autonomy supportive climate.

Goal orientation. For the variable task goal orientation neither the exclusion of the highest and lowest effect sizes $(g=1.118,95 \% \mathrm{CI}=$ 0.414 to $1.822, p=0.002)$ nor the exclusion of outliers $(g=0.763,95 \%$ $\mathrm{CI}=0.304$ to $1.222, p=0.01$ ) changed effect estimates; a large, significant Hedges' $g$ remained.

Additional file 3 shows the results of the subgroup analyses performed for CTs only. The direction of the results was similar to those presented in Table 4. Due to the small number of RCTs included in the various outcomes, the subgroup analyses were not repeated for RCTs.

\subsection{Secondary outcomes}

Of the 28 studies examining intervention effects in both motivational and PA outcomes, significant results in PA behavior were reported in 22 studies (Braga et al., 2017; Christodoulidis et al., 2001; Fairclough and Stratton, 2005; Fairclough et al., 2016; Fu et al., 2016; González-Cutre et al., 2018; Gorely et al., 2009; Harrington et al., 2018; Jamner et al., 2004; Lonsdale et al., 2013, Lonsdale et al., 2019; Meng et al., 2013; Meng and Keng, 2016; Miller et al., 2016; Moreno-Murcia and Sànchez-Latorre, 2016; Pardo et al., 2016; Salmon et al., 2008; Shannon et al., 2018; Smith et al., 2015; Wallhead, Garn, \& Vidoni, 2014; Wang et al., 2017; Yli-Piipari et al., 2018). Significant results in both motivational and PA outcomes were identified in 13 of these studies (Christodoulidis et al., 2001; Fairclough et al., 2016; González-Cutre et al., 2018; Gorely et al., 2009; ; Lonsdale et al., 2013, Lonsdale et al., 2019; Meng and Keng, 2016; Moreno-Murcia and Sànchez-Latorre, 2016; Pardo et al., 2016; Salmon et al., 2008; Shannon et al., 2018; Wallhead, Garn, \& Vidoni, 2014; Yli-Piipari et al., 2018). Intervention effects in favor of the IG were found for self-reported PA behavior (Christodoulidis et al., 2001; González-Cutre et al., 2018; Jamner et al., 2004; Moreno-Murcia and Sànchez-Latorre, 2016; Wallhead, Garn, \& Vidoni, 2014; Wang et al., 2017; Yli-Piipari et al., 2018), PA levels in-class (Braga et al., 2017; Fairclough and Stratton, 2005; Fairclough et al., 2016; Fu et al., 2016; Lonsdale et al., 2013, Lonsdale et al., 2019; Meng et al., 2013; Meng and Keng, 2016; Miller et al., 2016; Smith et al., 2015), during the school day (Fairclough et al., 2016; Shannon et al., 2018) and over several days (Gorely et al., 2009; Harrington et al., 2018; Lonsdale et al., 2019; Pardo et al., 2016; Salmon et al., 2008). Along with increasing in-class PA levels, two studies were also effective in decreasing in-class sedentary time (Lonsdale et al., 2013, Lonsdale et al., 2019). 
Table 3

Summary of effect size statistic and heterogeneity for all motivational outcome analyses.

\begin{tabular}{|c|c|c|c|c|c|c|c|c|c|c|}
\hline \multirow[t]{2}{*}{ Variables } & \multirow[t]{2}{*}{ Study design } & \multicolumn{6}{|c|}{ Effect size statistic } & \multicolumn{2}{|c|}{ Heterogeneity statistic } & \multirow{2}{*}{$\frac{\text { Publication bias }}{p^{\mathrm{A}}}$} \\
\hline & & $\mathrm{k}$ & $g$ & SE & $95 \% \mathrm{CI}$ & $\mathrm{Z}$ & $\mathrm{p}$ & $\mathrm{Q}$ & $\mathrm{I}^{2}$ & \\
\hline \multirow[t]{3}{*}{ Enjoyment } & Overall & 17 & 0.310 & 0.118 & $0.080,0.541$ & 2.641 & 0.008 & 130.405 & 87.730 & 0.096 \\
\hline & $\mathrm{CT}$ & 14 & 0.404 & 0.131 & $0.148,0.660$ & 3.092 & 0.002 & 117.080 & 88.896 & \\
\hline & RCT & 3 & -0.216 & 0.137 & $-0.484,0.051$ & -1.585 & 0.113 & 2.380 & 15.979 & \\
\hline \multicolumn{11}{|l|}{ Basic psychological needs } \\
\hline \multirow[t]{3}{*}{ Perceived autonomy } & Overall & 18 & 0.152 & 0.071 & $0.013,0.290$ & 2.144 & 0.032 & 55.426 & 69.328 & 0.368 \\
\hline & CT & 14 & 0.196 & 0.089 & $0.020,0.371$ & 2.189 & 0.029 & 45.201 & 71.240 & \\
\hline & RCT & 4 & -0.011 & 0.052 & $-0.114,0.091$ & -0.219 & 0.827 & 0.783 & 0.000 & \\
\hline \multirow[t]{3}{*}{ Perceived competence } & Overall & 28 & 0.080 & 0.167 & $-0.247,0.407$ & 0.480 & 0.631 & 778.120 & 96.530 & 0.133 \\
\hline & $\mathrm{CT}$ & 23 & 0.095 & 0.216 & $-0.327,0.518$ & 0.442 & 0.659 & 759.587 & 97.104 & \\
\hline & RCT & 5 & -0.009 & 0.052 & $-0.110,0.093$ & -0.166 & 0.868 & 2.104 & 0.000 & \\
\hline \multirow[t]{3}{*}{ Perceived relatedness } & Overall & 19 & 0.064 & 0.076 & $-0.084,0.212$ & 0.844 & 0.398 & 74.157 & 75.727 & 0.643 \\
\hline & CT & 13 & 0.140 & 0.112 & $-0.080,0.361$ & 1.250 & 0.211 & 65.476 & 81.673 & \\
\hline & RCT & 6 & -0.043 & 0.088 & $-0.215,0.129$ & -0.485 & 0.628 & 8.569 & 41.653 & \\
\hline \multicolumn{11}{|l|}{ Motivation } \\
\hline \multirow[t]{3}{*}{ Amotivation } & Overall & 6 & -0.208 & 0.116 & $-0.436,0.019$ & -1.794 & 0.073 & 23.509 & 78.732 & 0.597 \\
\hline & CT & 5 & -0.241 & 0.173 & $-0.580,0.097$ & -1.396 & 0.163 & 23.506 & 82.983 & \\
\hline & RCT & 1 & -0.155 & 0.057 & $-0.266,-0.045$ & -2.751 & 0.006 & 0.000 & 0.000 & \\
\hline External Regulation & CT & 5 & -0.114 & 0.256 & $-0.616,0.389$ & -0.443 & 0.657 & 51.822 & 92.281 & 0.329 \\
\hline Introjected Regulation & $\mathrm{CT}$ & 4 & 0.102 & 0.102 & $-0.098 ; 0.301$ & 1.000 & 0.317 & 4.195 & 28.488 & 0.039 \\
\hline \multirow[t]{3}{*}{ Identified Regulation } & Overall & 7 & 0.378 & 0.172 & $0.041,0.714$ & 2.199 & 0.028 & 59.177 & 89.861 & 0.101 \\
\hline & $\mathrm{CT}$ & 6 & 0.456 & 0.202 & $0.060,0.851$ & 2.258 & 0.024 & 45.301 & 88.963 & \\
\hline & RCT & 1 & -0.024 & 0.082 & $-0.185,0.137$ & -0.294 & 0.769 & 0.000 & 0.000 & \\
\hline \multirow[t]{3}{*}{ Intrinsic Motivation } & Overall & 11 & 0.419 & 0.170 & $0.085,0.752$ & 2.460 & 0.014 & 108.884 & 90.816 & 0.259 \\
\hline & $\mathrm{CT}$ & 10 & 0.471 & 0.190 & $0.099,0.842$ & 2.483 & 0.013 & 90.076 & 90.008 & \\
\hline & RCT & 1 & -0.033 & 0.090 & $-0.209,0.143$ & -0.371 & 0.710 & 0.000 & 0.000 & \\
\hline \multirow[t]{3}{*}{ SDI } & Overall & 8 & 0.672 & 0.286 & $0.112,1.232$ & 2.353 & 0.019 & 111.137 & 93.701 & 0.088 \\
\hline & CT & 5 & 1.161 & 0.429 & $0.321,2.001$ & 2.708 & 0.007 & 98.572 & 95.942 & \\
\hline & RCT & 3 & -0.061 & 0.131 & $-0.317,0.196$ & -0.463 & 0.643 & 0.055 & 0.000 & \\
\hline \multirow[t]{3}{*}{ RAI } & Overall & 5 & 0.086 & 0.113 & $-0.135,0.307$ & 0.765 & 0.444 & 12.961 & 69.138 & 0.515 \\
\hline & $\mathrm{CT}$ & 4 & -0.003 & 0.008 & $-0.160,0.153$ & -0.043 & 0.996 & 3.660 & 18.043 & \\
\hline & RCT & 1 & 0.367 & 0.062 & $0.019,0.264$ & 2.270 & 0.023 & 0.000 & 0.000 & \\
\hline \multicolumn{11}{|l|}{ Motivational climate } \\
\hline Task/Mastery & $\mathrm{CT}$ & 9 & 0.254 & 0.080 & $0.097,0.411$ & 3.177 & 0.001 & 20.258 & 60.509 & 0.851 \\
\hline Ego/Performance & $\mathrm{CT}$ & 9 & -0.438 & 0.106 & $-0.646,-0.230$ & -4.121 & 0.000 & 38.182 & 79.048 & 0.589 \\
\hline \multirow[t]{3}{*}{ Autonomy supportive climate } & Overall & 9 & 0.262 & 0.122 & $0.022,0.501$ & 2.140 & 0.032 & 62.477 & 87.195 & 0.521 \\
\hline & $\mathrm{CT}$ & 4 & 0.180 & 0.231 & $-0.273,0.634$ & 0.778 & 0.436 & 32.352 & 90.727 & \\
\hline & RCT & 5 & 0.340 & 0.147 & $0.053,0.628$ & 2.318 & 0.020 & 22.501 & 82.223 & \\
\hline \multicolumn{11}{|l|}{ Goal Orientation } \\
\hline Task & $\mathrm{CT}$ & 8 & 1.370 & 0.345 & $0.694,2.045$ & 3.972 & 0.000 & 96.124 & 92.718 & 0.024 \\
\hline Ego & $\mathrm{CT}$ & 8 & -0.188 & 0.071 & $-0.327,-0.050$ & -2.668 & 0.008 & 7.325 & 4.438 & 0.764 \\
\hline
\end{tabular}

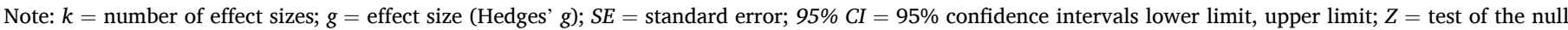
hypothesis; $I^{2}=$ total variance explained by moderators; ${ }^{\text {A }}$ two-tailed p-value (Eggers' test); SDI = self-determination index; RAI = relative autonomy index.

\subsection{Certainty of evidence}

The certainty of evidence was assessed in accordance with GRADE for the meta-analyzed outcomes enjoyment, basic psychological needs, motivation, motivational climate, and goal orientation (Table 5). The certainty of evidence was initially set at high for RCTs and CTs and downgraded one point for each item rated 'serious' and two points for each item rated 'very serious'; the optimal information size was set at $\mathrm{n}$ $>400$.

Every meta-analyzed outcome was downgraded one, two or three levels. Reasons for downgrading were serious risk of bias, serious or very serious inconsistency, serious imprecision and/or publication bias. Moderate certainty of evidence was shown for ego/performance climate and ego goal orientation. Low certainty of evidence was determined in autonomy supportive climate, task/mastery climate, RAI, SDI, amotivation, perceived autonomy, and enjoyment. Very low certainty of evidence was found for perceived competence and relatedness, external regulation, introjected and identified regulation, intrinsic motivation, and task goal orientation. There was no indication of indirectness regarding the population, intervention, comparator, or outcome in any variable. Publication bias was strongly suspected in the outcomes introjected regulation and task goal orientation. The explanations for each judgement can be found in Table 5 .

\section{Discussion}

The aim of this study was to provide information on the effects of school-based PA interventions on students' motivation towards PA. Secondarily, effects on PA behavior were analyzed.

\subsection{Main findings-primary outcomes}

The results of the meta-analyses on motivational outcomes indicated that the overall pooled effect size of school-based PA interventions was small to moderate, and statistically significant, for enjoyment, perceived autonomy, identified regulation, intrinsic motivation, motivational climate and ego goal orientation. Significant moderate to large effects were also identified for SDI and task goal orientation. Non-significant effect estimates were found in the meta-analyses of perceived competence, perceived relatedness, amotivation, external regulation, introjected regulation and RAI. A further inspection of the data revealed large heterogeneity across studies in the meta-analyzed outcomes except for the outcomes ego goal orientation and introjected regulation.

These results are in line with previous findings on the effectiveness of PA interventions implemented in the school setting on motivational outcomes. For example, the meta-analysis by Burns et al. (2017) revealed small to moderate effects of school-based PA interventions on enjoyment . Braithwaite et al. (2011) found that PA interventions 
Table 4

Moderator statistics.

\begin{tabular}{|c|c|c|c|c|c|c|c|c|c|}
\hline \multirow[t]{2}{*}{ Variables } & & \multicolumn{6}{|c|}{ Effect size statistic } & \multicolumn{2}{|c|}{ Heterogeneity statistic } \\
\hline & & $\mathrm{k}$ & $\mathrm{g}$ & SE & $95 \% \mathrm{CI}$ & $\mathrm{Z}$ & $\mathrm{p}$ & $\mathrm{Q}$ & $\mathrm{I}^{2}$ \\
\hline \multirow[t]{12}{*}{ Enjoyment } & & 17 & 0.310 & 0.118 & $0.080,0.541$ & 2.641 & 0.008 & 130.405 & 87.730 \\
\hline & High/Low & 15 & 0.261 & 0.104 & $0.058,0.464$ & 2.515 & 0.012 & 79.383 & 82.364 \\
\hline & Outlier & 13 & 0.202 & 0.077 & $0.051,0.354$ & 2.618 & 0.009 & 28.353 & 57.677 \\
\hline & Theory-based & & & & & & & $4.497^{\mathrm{A} *}$ & \\
\hline & Yes & 12 & 0.465 & 0.136 & $0.199,0.732$ & 3.427 & 0.001 & 102.957 & 89.306 \\
\hline & No & 5 & -0.064 & 0.210 & $-0.475,0.347$ & -0.307 & 0.759 & 9.503 & 57.909 \\
\hline & Age & & & & & & & $2.060^{\mathrm{A}}$ & \\
\hline & Children & 7 & 0.114 & 0.185 & $-0.249,0.478$ & 2.534 & 0.537 & 62.357 & 90.378 \\
\hline & Adolescents & 10 & 0.471 & 0.166 & $0.147,0.796$ & 2.846 & 0.004 & 63.102 & 85.737 \\
\hline & Study design & & & & & & & $3.209^{\mathrm{A}}$ & \\
\hline & $\mathrm{CT}$ & 14 & 0.402 & 0.127 & $0.153,0.651$ & 2.167 & 0.002 & 117.080 & 88.896 \\
\hline & RCT & 3 & -0.157 & 0.285 & $-0.716,0.402$ & -0.551 & 0.582 & 2.380 & 15.979 \\
\hline \multicolumn{10}{|l|}{ Basic psychological needs } \\
\hline \multirow[t]{8}{*}{ Perceived autonomy } & & 18 & 0.152 & 0.071 & $0.013,0.290$ & 2.144 & 0.032 & 55.426 & 69.328 \\
\hline & High/Low & 16 & 0.137 & 0.061 & $0.017,0.257$ & 2.245 & 0.025 & 34.199 & 56.139 \\
\hline & Age & & & & & & & $4.718^{\mathrm{A} *}$ & \\
\hline & Children & 4 & 0.407 & 0.134 & $0.144,0.671$ & 3.031 & 0.002 & 1.713 & 0.000 \\
\hline & Adolescents & 14 & 0.074 & 0.074 & $-0.070,0.219$ & 1.006 & 0.314 & 40.602 & 67.982 \\
\hline & Study design & & & & & & & $1.320^{\mathrm{A}}$ & \\
\hline & CT & 14 & 0.196 & 0.081 & $0.037,0.355$ & 2.418 & 0.016 & 45.201 & 71.240 \\
\hline & RCT & 4 & 0.001 & 0.149 & $-0.291,0.294$ & 0.008 & 0.934 & 0.783 & 0.000 \\
\hline \multirow[t]{12}{*}{ Perceived competence } & & 28 & 0.080 & 0.167 & $-0.247,0.407$ & 0.480 & 0.631 & 778.120 & 96.530 \\
\hline & High/Low & 26 & 0.114 & 0.069 & $-0.022,0.250$ & 1.648 & 0.099 & 100.550 & 75.235 \\
\hline & Outlier & 24 & 0.095 & 0.048 & $0.001,0.189$ & 1.973 & 0.048 & 39.405 & 41.632 \\
\hline & Theory-based & & & & & & & $0.016^{\mathrm{A}}$ & \\
\hline & Yes & 26 & 0.075 & 0.175 & $-0.268,0.417$ & 0.426 & 0.670 & 767.923 & 96.744 \\
\hline & No & 2 & 0.156 & 0.630 & $-1.078,1.391$ & 0.248 & 0.804 & 2.112 & 52.647 \\
\hline & Age & & & & & & & $1.550^{\mathrm{A}}$ & \\
\hline & Children & 7 & -0.079 & 0.322 & $-0.710,0.552$ & -0.245 & 0.806 & 574.947 & 98.956 \\
\hline & Adolescents & 20 & 0.179 & 0.191 & $-0.196,0.554$ & 0.937 & 0.349 & 57.411 & 66.906 \\
\hline & Study design & & & & & & & $0.015^{\mathrm{A}}$ & \\
\hline & CT & 23 & 0.093 & 0.201 & $-0.300,0.486$ & 0.464 & 0.643 & 759.587 & 97.104 \\
\hline & RCT & 5 & 0.035 & 0.433 & $-0.813,0.884$ & 0.081 & 0.935 & 2.104 & 0.000 \\
\hline Perceived relatedness & & 19 & 0.064 & 0.076 & $-0.084,0.212$ & 0.844 & 0.398 & 74.157 & 75.727 \\
\hline & High/Low & 17 & 0.032 & 0.067 & $-0.100,0.164$ & 0.476 & 0.634 & 49.518 & 67.689 \\
\hline & Outlier & 16 & 0.020 & 0.057 & $-0.093,0.132$ & 0.340 & 0.734 & 29.420 & 49.014 \\
\hline & Theory-based & & & & & & & $1.623^{\mathrm{A}}$ & \\
\hline & Yes & 17 & 0.097 & 0.080 & $-0.060,0.254$ & 1.215 & 0.224 & 68.960 & 76.798 \\
\hline & No & 2 & -0.214 & 0.231 & $-0.666,0.238$ & -0.927 & 0.354 & 1.148 & 12.884 \\
\hline & Age & & & & & & & $0.008^{\mathrm{A}}$ & \\
\hline & Children & 6 & 0.075 & 0.138 & $-0.195,0.346$ & 0.545 & 0.586 & 11.382 & 56.070 \\
\hline & Adolescents & 13 & 0.061 & 0.095 & $-0.126,0.248$ & 0.636 & 0.525 & 62.630 & 80.840 \\
\hline & Study design & & & & & & & $1.395^{\mathrm{A}}$ & \\
\hline & $\mathrm{CT}$ & 13 & 0.133 & 0.099 & $-0.061,0.328$ & 1.342 & 0.180 & 65.476 & 81.673 \\
\hline & RCT & 6 & -0.075 & 0.145 & $-0.360,0.210$ & -0.514 & 0.607 & 8.569 & 41.653 \\
\hline Motivation & & & & & & & & & \\
\hline Amotivation & & 6 & -0.208 & 0.116 & $-0.436,0.019$ & -1.794 & 0.073 & 23.509 & 78.732 \\
\hline & High/Low & 4 & -0.147 & 0.047 & $-0.240,-0.054$ & -3.106 & 0.002 & 1.726 & 0.000 \\
\hline & Outlier & 5 & -0.111 & 0.053 & $-0.215,-0.007$ & -2.083 & 0.037 & 4.633 & 13.654 \\
\hline & Study design & & & & & & & $0.222^{\mathrm{A}}$ & \\
\hline & $\mathrm{CT}$ & 5 & -0.241 & 0.173 & $-0.580,0.097$ & -1.396 & 0.163 & 23.506 & 82.983 \\
\hline & RCT & 1 & -0.155 & 0.057 & $-0.266,-0.045$ & -2.751 & 0.006 & 0.000 & 0.000 \\
\hline External Regulation & & 5 & -0.114 & 0.256 & $-0.616,0.389$ & -0.443 & 0.657 & 51.822 & 92.281 \\
\hline & High/Low & 3 & -0.009 & 0.117 & $-0.237,0.220$ & -0.074 & 0.941 & 3.494 & 42.753 \\
\hline & Outlier & 4 & 0.097 & 0.139 & $-0.176,0.370$ & 0.697 & 0.486 & 8.407 & 64.315 \\
\hline Introjected Regulation & & 4 & 0.102 & 0.102 & $-0.098 ; 0.301$ & 1.000 & 0.317 & 4.195 & 28.488 \\
\hline & High/Low & 2 & 0.144 & 0.125 & $-0.101,0.388$ & 1.154 & 0.249 & 0.394 & 0.000 \\
\hline Identified Regulation & & 7 & 0.378 & 0.172 & $0.041,0.714$ & 2.199 & 0.028 & 59.177 & 89.861 \\
\hline & High/Low & 5 & 0.291 & 0.153 & $-0.008,0.591$ & 1.907 & 0.057 & 24.414 & 63.616 \\
\hline & Outlier & 6 & 0.218 & 0.137 & $-0.050,0.485$ & 1.594 & 0.111 & 29.507 & 83.282 \\
\hline & Study design & & & & & & & $4.850^{\mathrm{A} *}$ & \\
\hline & $\mathrm{CT}$ & 6 & 0.456 & 0.202 & $0.060,0.851$ & 2.258 & 0.024 & 45.301 & 88.963 \\
\hline & RCT & 1 & -0.024 & 0.082 & $-0.185,0.137$ & -0.294 & 0.769 & 0.000 & 0.000 \\
\hline Intrinsic Motivation & & 11 & 0.419 & 0.170 & $0.085,0.752$ & 2.460 & 0.014 & 108.884 & 90.816 \\
\hline & High/Low & 9 & 0.325 & 0.148 & $0.035,0.614$ & 2.198 & 0.028 & 60.751 & 66.831 \\
\hline & Outlier & 9 & 0.339 & 0.145 & $0.055,0.622$ & 2.343 & 0.019 & 50.306 & 84.096 \\
\hline & Theory-based & & & & & & & $0.005^{\mathrm{A}}$ & \\
\hline & Yes & 10 & 0.421 & 0.179 & $0.071,0.772$ & 2.355 & 0.019 & 108.820 & 91.729 \\
\hline & No & 1 & 0.391 & 0.385 & $-0.364,1.145$ & 1.015 & 0.310 & 0.000 & 0.000 \\
\hline & Age & & & & & & & $0.571^{\mathrm{A}}$ & \\
\hline & Children & 6 & 0.290 & 0.201 & $-0.104,0.683$ & 1.442 & 0.149 & 35.758 & 86.017 \\
\hline & Adolescents & 5 & 0.557 & 0.324 & $-0.057,1.212$ & 1.784 & 0.074 & 73.049 & 94.524 \\
\hline & Study design & & & & & & & $5.775^{\mathrm{A} *}$ & \\
\hline
\end{tabular}


Table 4 (continued)

\begin{tabular}{|c|c|c|c|c|c|c|c|c|c|}
\hline \multirow[t]{2}{*}{ Variables } & & \multicolumn{6}{|c|}{ Effect size statistic } & \multicolumn{2}{|c|}{ Heterogeneity statistic } \\
\hline & & $\mathrm{k}$ & $\mathrm{g}$ & SE & $95 \% \mathrm{CI}$ & $\mathrm{Z}$ & $\mathrm{p}$ & $\mathrm{Q}$ & $\mathrm{I}^{2}$ \\
\hline & CT & 10 & 0.471 & 0.190 & $0.099,0.842$ & 2.483 & 0.013 & 90.076 & 90.008 \\
\hline & RCT & 1 & -0.033 & 0.090 & $-0.209,0.143$ & -0.371 & 0.710 & 0.000 & 0.000 \\
\hline \multirow[t]{5}{*}{ SDI } & & 8 & 0.672 & 0.286 & $0.112,1.232$ & 2.353 & 0.019 & 111.137 & 93.701 \\
\hline & High/Low & 6 & 0.404 & 0.237 & $-0.062,0.869$ & 1.700 & 0.089 & 41.348 & 87.908 \\
\hline & Design & & & & & & & $7.430^{\mathrm{A} * *}$ & \\
\hline & CT & 5 & 1.161 & 0.429 & $0.321,2.001$ & 2.708 & 0.007 & 98.572 & 95.942 \\
\hline & RCT & 3 & -0.061 & 0.131 & $-0.317,0.196$ & -0.463 & 0.643 & 0.055 & 0.000 \\
\hline \multirow[t]{5}{*}{ RAI } & & 5 & 0.086 & 0.113 & $-0.135,0.307$ & 0.765 & 0.444 & 12.961 & 69.138 \\
\hline & High/Low & 3 & 0.005 & 0.089 & $-0.169,0.179$ & 0.052 & 0.958 & 3.203 & 37.567 \\
\hline & Study design & & & & & & & $8.434^{\mathrm{A} * *}$ & \\
\hline & CT & 4 & -0.003 & 0.008 & $-0.160,0.153$ & -0.043 & 0.996 & 3.660 & 18.043 \\
\hline & RCT & 1 & 0.367 & 0.062 & $0.019,0.264$ & 2.270 & 0.023 & 0.000 & 0.000 \\
\hline \multicolumn{10}{|l|}{ Motivational climate } \\
\hline \multirow[t]{2}{*}{ Task/Mastery } & & 9 & 0.254 & 0.080 & $0.097,0.411$ & 3.177 & 0.001 & 20.258 & 60.509 \\
\hline & High/Low & 7 & 0.259 & 0.077 & $0.109,0.409$ & 3.386 & 0.001 & 13.098 & 54.193 \\
\hline \multirow[t]{3}{*}{ Ego/Performance } & & 9 & -0.438 & 0.106 & $-0.646,-0.230$ & -4.121 & 0.000 & 38.182 & 79.048 \\
\hline & High/Low & 7 & -0.371 & 0.045 & $-0.460,-0.282$ & -8.180 & 0.000 & 1.951 & 0.000 \\
\hline & Outlier & 8 & -0.355 & 0.045 & $-0.442,-0.267$ & -7.924 & 0.000 & 6.919 & 0.000 \\
\hline \multirow[t]{9}{*}{ Autonomy supportive climate } & & 9 & 0.262 & 0.122 & $0.022,0.501$ & 2.140 & 0.032 & 62.477 & 87.195 \\
\hline & High/Low & 7 & 0.280 & 0.121 & $0.043,0.516$ & 2.316 & 0.021 & 34.131 & 82.421 \\
\hline & Outlier & & 0.337 & 0.119 & $0.104,0.570$ & 2.833 & 0.005 & 42.270 & 83.440 \\
\hline & Age & & & & & & & $9.486^{\mathrm{A} * *}$ & \\
\hline & Children & 2 & 0.605 & 0.088 & $0.433,0.778$ & 6.871 & 0.000 & 0.882 & 0.000 \\
\hline & Adolescents & 7 & 0.138 & 0.123 & $-0.103,0.380$ & 1.121 & 0.262 & 33.931 & 82.317 \\
\hline & Study design & & & & & & & $0.427^{\mathrm{A}}$ & \\
\hline & CT & 4 & 0.172 & 0.190 & $-0.201,0.545$ & 0.904 & 0.366 & 32.352 & 90.727 \\
\hline & RCT & 5 & 0.341 & 0.175 & $-0.003,0.685$ & 1.943 & 0.052 & 22.501 & 82.223 \\
\hline \multicolumn{10}{|l|}{ Goal Orientation } \\
\hline \multirow{3}{*}{ Task } & & 8 & 1.370 & 0.345 & $0.694,2.045$ & 3.972 & 0.000 & 96.124 & 92.718 \\
\hline & High/Low & 6 & 1.118 & 0.359 & $0.414,1.822$ & 3.113 & 0.002 & 50.449 & 90.089 \\
\hline & Outlier & 4 & 0.763 & 0.234 & $0.304,1.222$ & 3.260 & 0.001 & 6.101 & 50.824 \\
\hline \multirow[t]{2}{*}{ Ego } & & 8 & -0.188 & 0.071 & $-0.327,-0.050$ & -2.668 & 0.008 & 7.325 & 4.438 \\
\hline & High/Low & 6 & -0.167 & 0.068 & $-0.300,-0.034$ & -2.460 & 0.014 & 1.261 & 0.000 \\
\hline
\end{tabular}

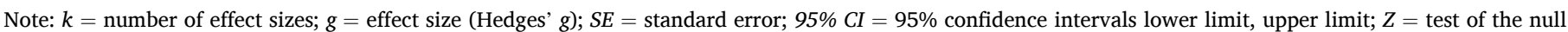

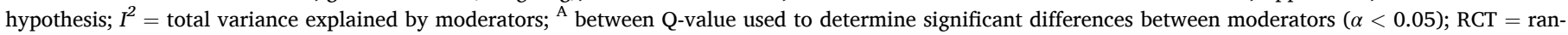
domized controlled trial; $\mathrm{CT}=$ controlled trial; $\mathrm{SDI}=$ self-determination Index; $\mathrm{RAI}=$ relative autonomy index; $*$ indicates $p<0.05, * * p<0.01$.

conducted exclusively in PE positively affected students' enjoyment, task orientation, and perception of mastery and performance climate. The magnitude of meta-analyzed effects was small to moderate. While Braithwaite et al. (2011) only focused on motivational climate interventions using the TARGET approach in PE, Burns et al. (2017) included studies that focused on student-centered approaches, where students were given choices and incorporated in decision-making; e.g. by using the Sport Education Model. The intervention components were implemented during the regular school day or after school (Burns et al., 2017). In contrast, our review and meta-analysis included studies that implemented established teaching approaches (e.g. TARGET, TGfU, Sport Education Model) as well as self-designed teaching approaches during the regular school day.

For a more comprehensive overview of intervention strategies used across the examined studies, we coded the intervention components using the standardized taxonomy by Michie et al., 2013). The analysis of BCTs showed that a variety of techniques within 12 of 16 clusters was used among the studies. However, regardless of whether the examined outcome was enjoyment, goal orientation, motivational climate or self-determined motivation, similar techniques were frequently applied and little variation was observed. In the meta-analyzed studies, the most frequently applied techniques were identified within the clusters 'Shaping knowledge' (e.g. instruction on how to perform behavior), 'Comparison of behavior' (e.g. demonstration or social comparison of behavior), 'Repetition and substitution' (e.g. behavioral practice/rehearsal), and 'Antecedents' (e.g. restructuring physical/social environment). Studies aiming at goal orientation also frequently included 'Goals and planning' strategies, whereas studies aiming at motivational climate additionally employed techniques within the clusters 'Feedback and monitoring' and 'Natural consequences' (e.g. Information about health consequences). Due to the numerous techniques used and the variety of outcomes measured within and across the studies, it is not possible to clearly identify, which individual BCT is essential and truly effective in changing a certain motivational outcome. Further research is needed to clarify which techniques are required to change a particular motivational outcome and thus to develop more effective interventions.

Similar to the meta-analyses by Burns et al. (2017) and Braithwaite et al. (2011), the overall pooled effects were affected by large heterogeneity, giving reason to further explore these in the subgroup analyses. Removing outliers and studies with the highest/lowest effect estimates reduced heterogeneity in most outcomes. Additionally, heterogeneity was explained by study characteristics (i.e. use of a theoretical framework), participant characteristics (i.e. age group of participants), and/or study design in the outcomes enjoyment, basic psychological needs, autonomy supportive climate, and intrinsic motivation. Significant differences between groups of moderators were found for the use of a theoretical framework in the outcome enjoyment $(p<0.05)$ and participants' age in the outcomes perceived autonomy $(p<0.05)$ and autonomy supportive climate $(p<0.01)$. Regarding the study design, significant differences between RCTs and CTs were confirmed in four out of 16 outcomes, i.e. identified regulation, intrinsic motivation (both $p<$ 0.05), SDI, and RAI (both $p<0.01$ ). However, the confidence in these findings is low since only one RCT was included in the analyses of these variables. This also holds true for SDI, where the underlying RCT by Lonsdale et al. (2013) included three intervention groups, therefore three separate analyses were performed, resulting in $k=3$. These findings support the combined analysis of CTs and RCTs in this study. Overall, the small number of individual effect sizes for many meta-analyzed outcomes limited the number of examined moderators in the subgroup analyses. Other study features such as study duration or 


\begin{tabular}{|c|c|c|c|c|c|c|c|c|}
\hline \multicolumn{7}{|c|}{ Certainty assessment } & \multirow[t]{2}{*}{ Impact } & \multirow[t]{2}{*}{ Certainty } \\
\hline $\begin{array}{l}\text { № of } \\
\text { studies }\end{array}$ & $\begin{array}{l}\text { Study } \\
\text { design }\end{array}$ & $\begin{array}{l}\text { Risk of } \\
\text { bias }\end{array}$ & Inconsistency & Indirectness & Imprecision & Other considerations & & \\
\hline \multicolumn{9}{|c|}{ Enjoyment (follow up: range 3 weeks to 3 school years; assessed with: questionnaire) } \\
\hline \multirow[t]{2}{*}{17} & $\begin{array}{l}\text { RCT (n } \\
=3) \text {, }\end{array}$ & serious $^{\text {a }}$ & serious $^{\mathrm{b}}$ & not serious & not serious & none & $\begin{array}{l}\text { Meta-analysis revealed an overall small to moderate effect (Hedges' } g=0.310,95 \% \mathrm{CI}=0.080 ; 0.541) \text {. } \\
\text { Subgroup analyses identified small to moderate and significant effect estimates for theory-based studies }(\mathrm{k}=\end{array}$ & $\oplus \oplus \bigcirc \bigcirc$ \\
\hline & $\begin{array}{l}\mathrm{CT}(\mathrm{n}= \\
14)\end{array}$ & & & & & & 12) $(p<0.01)$, studies in adolescents $(k=10)(p<0.01)$ and in CTs $(k=14)(p<0.01)$ & LOW \\
\hline \multicolumn{9}{|c|}{ Perceived autonomy (follow up: range 4 weeks to 7-8 months; assessed with: questionnaire) } \\
\hline \multirow[t]{2}{*}{18} & $\begin{array}{l}\mathrm{RCT}(\mathrm{n}= \\
4)\end{array}$ & serious $^{\text {a }}$ & serious ${ }^{c}$ & not serious & not serious & none & $\begin{array}{l}\text { Meta-analysis revealed an overall small effect (Hedges' } g=0.152,95 \% \mathrm{CI}=0.013 ; 0.290) \text {. Subgroup } \\
\text { analyses identified small to moderate and significant effect estimates for studies in children }(\mathrm{k}=4)(\mathrm{p}<\end{array}$ & $\oplus \oplus \bigcirc \bigcirc$ \\
\hline & $\begin{array}{l}\mathrm{CT}(\mathrm{n}= \\
14)\end{array}$ & & & & & & $0.01)$ and in CTs $(k=14)(\mathrm{p}<0.05)$ & LOW \\
\hline \multicolumn{9}{|c|}{ Perceived competence (follow up: range 1 days to 1 school year; assessed with: questionnaire) } \\
\hline \multirow[t]{2}{*}{28} & $\begin{array}{l}\text { RCT( }(n= \\
5),\end{array}$ & serious $^{\mathrm{a}}$ & serious $^{\mathrm{d}}$ & not serious & serious ${ }^{\mathrm{e}}$ & none & Meta-analysis revealed no significant effect (Hedges' $g=0.080,95 \% \mathrm{CI}=-0.247$; 0.407) & $\oplus \bigcirc \bigcirc \bigcirc$ \\
\hline & $\begin{array}{l}\mathrm{CT}(\mathrm{n}= \\
13)\end{array}$ & & & & & & & VERY LOW \\
\hline \multicolumn{9}{|c|}{ Perceived relatedness (follow up: range 4 weeks to 3 school years; assessed with: questionnaire) } \\
\hline \multirow[t]{2}{*}{19} & $\begin{array}{l}\text { RCT }(\mathrm{n} \\
=6)\end{array}$ & serious $^{\text {a }}$ & serious ${ }^{f}$ & not serious & serious ${ }^{\mathrm{e}}$ & none & Meta-analysis revealed no significant effect (Hedges' $g=0.064,95 \% \mathrm{CI}=-0.084 ; 0.212$ ). & $\oplus \bigcirc \bigcirc \bigcirc$ \\
\hline & $\begin{array}{l}\mathrm{CT}(\mathrm{n}= \\
13)\end{array}$ & & & & & & & VERY LOW \\
\hline \multicolumn{9}{|c|}{ Amotivation (follow up: range 5 weeks to 1 school year; assessed with questionnaire) } \\
\hline \multirow[t]{2}{*}{6} & $\begin{array}{l}\text { RCT }(\mathrm{n} \\
=1)\end{array}$ & serious $^{\text {a }}$ & not serious ${ }^{\mathrm{g}}$ & not serious & serious ${ }^{\mathrm{e}}$ & none & Meta-analysis revealed no effect (Hedges' $g=-0.208,95 \% \mathrm{CI}-0.436,0.019$ ). & $\oplus \oplus \bigcirc \bigcirc$ \\
\hline & $\begin{array}{l}\text { CT }(n= \\
5)\end{array}$ & & & & & & & LOW \\
\hline \multicolumn{9}{|c|}{ External regulation (follow up: range 5 weeks to 13 weeks; assessed with questionnaire) } \\
\hline 5 & $\begin{array}{l}\text { CT }(\mathrm{n}= \\
\text { 5) }\end{array}$ & serious $^{\text {a }}$ & serious $^{\mathrm{h}}$ & not serious & serious ${ }^{\mathrm{e}}$ & none & Meta-analysis revealed no effect (Hedges' $g=-0.114,95 \% \mathrm{CI}=-0.616 ; 0.389$ ). & $\begin{array}{l}\oplus \bigcirc \bigcirc \bigcirc \\
\text { VERY LOW }\end{array}$ \\
\hline \multicolumn{9}{|c|}{ Introjected regulation (follow up: range 6 weeks to 13 weeks; assessed with questionnaire) } \\
\hline 4 & $\begin{array}{l}\text { CT }(n= \\
\text { 4) }\end{array}$ & serious $^{\mathrm{a}}$ & not serious & not serious & serious ${ }^{\mathrm{e}}$ & $\begin{array}{l}\text { publication bias } \\
\text { strongly suspected }\end{array}$ & Meta-analysis revealed no effect (Hedges' $g=0.102,95 \% \mathrm{CI}=-0.098 ; 0.301$ ). & $\begin{array}{l}\oplus \bigcirc \bigcirc \bigcirc \\
\text { VERY LOW }\end{array}$ \\
\hline \multicolumn{9}{|c|}{ Identified regulation (follow up: range 5 weeks to 13 weeks; assessed with: questionnaire) } \\
\hline \multirow[t]{2}{*}{7} & $\begin{array}{l}\text { RCT }(\mathrm{n} \\
=1)\end{array}$ & serious $^{\text {a }}$ & very serious ${ }^{i}$ & not serious & not serious & none & $\begin{array}{l}\text { Meta-analysis revealed an overall moderate effect (Hedges' } g=0.378,95 \% \mathrm{CI}=0.041 ; 0.714) \text {. Subgroup } \\
\text { analysis identified moderate and significant effect estimates for CTs }(k=6)(p<0.05) \text {. }\end{array}$ & $\oplus \bigcirc \bigcirc \bigcirc$ \\
\hline & $\begin{array}{l}\text { CT }(\mathrm{n}= \\
6)\end{array}$ & & & & & & & VERY LOW \\
\hline \multicolumn{9}{|c|}{ Intrinsic motivation (follow up: range 4 weeks to 13 weeks; assessed with: questionnaire) } \\
\hline \multirow[t]{3}{*}{11} & $\begin{array}{l}\text { RCT }(\mathrm{n} \\
=1)\end{array}$ & serious $^{\text {a }}$ & very serious ${ }^{\mathrm{i}}$ & not serious & not serious & none & $\begin{array}{l}\text { Meta-analysis revealed an overall small to moderate effect (Hedges' } g=0.419,95 \% \mathrm{CI}=0.085 ; 0.752 \text { ). } \\
\text { Subgroup analyses identified small to moderate and significant effect estimates for theory-based studies ( } k=\end{array}$ & \\
\hline & $\begin{array}{l}\text { СТ }(\mathrm{n}= \\
10)\end{array}$ & & & & & & $10)$ and CTs $(k=1)(p<0.05)$ & VERY LOW \\
\hline & \multicolumn{8}{|c|}{ SDI (follow up: range 4 weeks to 13 weeks; assessed with: questionnaire) } \\
\hline \multirow[t]{2}{*}{8} & $\begin{array}{l}\text { RCT }(\mathrm{n} \\
=3)\end{array}$ & serious $^{\text {a }}$ & serious $^{\mathrm{i}}$ & not serious & not serious & none & $\begin{array}{l}\text { Meta-analysis revealed an overall moderate to large effect (Hedges' } g=0.672,95 \% \mathrm{CI}=0.112 ; 1.232) \text {. } \\
\text { Subgroup analvses identified large and significant effect estimates for CTs }(k=5)(p<0.01)\end{array}$ & $\oplus \oplus \bigcirc \bigcirc$ \\
\hline & $\begin{array}{l}\text { СT }(\mathrm{n}= \\
\text { 5) }\end{array}$ & & & & & & & LOW \\
\hline \multicolumn{9}{|c|}{ RAI (follow up: range 4 we } \\
\hline \multirow[t]{2}{*}{5} & $\begin{array}{l}\text { RCT (n } \\
=1)\end{array}$ & serious $^{\text {a }}$ & not serious $^{j}$ & not serious & serious ${ }^{\mathrm{e}}$ & None & $\begin{array}{l}\text { Meta-analysis revealed a non-significant effect (Hedges' } g=0.086,95 \% \mathrm{CI}=-0.135 ; 0.307) \text {. Subgroup } \\
\text { analyses identified a small to moderate and significant effect estimate for RCTs }(k=1)(p<0.05) \text {. }\end{array}$ & $\oplus \oplus \bigcirc \bigcirc$ \\
\hline & $\begin{array}{l}\text { CT }(n= \\
\text { 4) }\end{array}$ & & & & & & & LOW \\
\hline \multicolumn{9}{|c|}{ Task/Mastery climate (follow up: range 5 weeks to 12 months; assessed with: questionnaire) } \\
\hline 9 & & serious $^{\mathrm{a}}$ & serious $^{\mathrm{k}}$ & not serious & not serious & None & Meta-analysis revealed an overall small effect (Hedges' $g=0.254,95 \% \mathrm{CI}=0.097$; 0.411 ). & $\oplus \oplus \circ \bigcirc$ \\
\hline
\end{tabular}


Table 5 (continued)

\begin{tabular}{|c|c|c|c|c|c|c|c|c|}
\hline \multicolumn{7}{|c|}{ Certainty assessment } & \multirow[t]{2}{*}{ Impact } & \multirow[t]{2}{*}{ Certainty } \\
\hline $\begin{array}{l}\text { № of } \\
\text { studies }\end{array}$ & $\begin{array}{l}\text { Study } \\
\text { design }\end{array}$ & $\begin{array}{l}\text { Risk of } \\
\text { bias }\end{array}$ & Inconsistency & Indirectness & Imprecision & Other considerations & & \\
\hline & $\begin{array}{l}\text { CT }(\mathrm{n}= \\
\text { 9) }\end{array}$ & & & & & & & LOW \\
\hline \multicolumn{9}{|c|}{ Ego/Performance climate (follow up: range 5 weeks to 12 months; assessed with: questionnaire) } \\
\hline 9 & $\begin{array}{l}\mathrm{CT}(\mathrm{n}= \\
\text { 9) }\end{array}$ & serious $^{\text {a }}$ & not serious & not serious & not serious & None & Meta-analysis revealed an overall moderate effect (Hedges' $g=-0.438,95 \% \mathrm{CI}=-0.646 ;-0.230$ ). & $\begin{array}{l}\oplus \oplus \oplus \bigcirc \\
\text { MODERATE }\end{array}$ \\
\hline \multicolumn{9}{|c|}{ Autonomy supportive climate (follow up: range 4 weeks to 15 months; assessed with: questionnaire) } \\
\hline 9 & $\begin{array}{l}\text { CT }(n= \\
\text { 9) }\end{array}$ & serious $^{\text {a }}$ & serious $^{1}$ & not serious & not serious & None & $\begin{array}{l}\text { Meta-analysis revealed an overall small effect (Hedges' } g=0.262,95 \% \mathrm{CI}=0.022 ; 0.501) \text {. Subgroup } \\
\text { analysis identified a moderate and significant effect estimate for studies in children }(k=2)(p<0.01) \text {. }\end{array}$ & $\begin{array}{l}\oplus \oplus \bigcirc \bigcirc \\
\text { LOW }\end{array}$ \\
\hline \multicolumn{9}{|c|}{ Task goal orientation (follow up: range 2 weeks to 1 school year; assessed with: questionnaire) } \\
\hline 8 & $\begin{array}{l}\mathrm{CT}(\mathrm{n}= \\
\text { 8) }\end{array}$ & serious $^{\mathrm{a}}$ & serious $^{\mathrm{m}}$ & not serious & not serious & $\begin{array}{l}\text { publication bias } \\
\text { strongly suspected }\end{array}$ & Meta-analysis revealed an overall large effect (Hedges' $g=1.370,95 \% \mathrm{CI}=0.694 ; 2.045$ ). & $\begin{array}{l}\oplus \bigcirc \bigcirc \bigcirc \\
\text { VERY LOW }\end{array}$ \\
\hline \multicolumn{9}{|c|}{ Ego goal orientation (follow up: range 2 weeks to 1 school year; assessed with: questionnaire) } \\
\hline 8 & $\begin{array}{l}\mathrm{CT}(\mathrm{n}= \\
8)\end{array}$ & serious $^{\mathrm{a}}$ & not serious & not serious & not serious & None & Meta-analysis revealed an overall small effect (Hedges' $g=-0.188,95 \% \mathrm{CI}=-0.327 ;-0.050$ ). & $\begin{array}{l}\oplus \oplus \oplus \bigcirc \\
\text { MODERATE }\end{array}$ \\
\hline
\end{tabular}

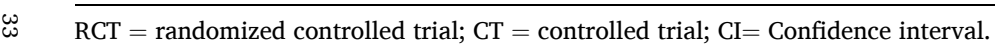

Explanations.

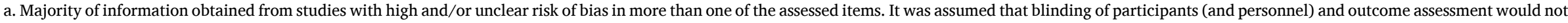
significantly affect the intervention results, therefore, risk of bias was considered serious and only downgraded one point.

b. Heterogeneity was largely explained in RCTs $(\mathrm{k}=3$ ) but remaining subgroups were affected by substantial heterogeneity. Inconsistency was downgraded one point.

c. Heterogeneity was not present in children $(\mathrm{k}=4)$ and in RCTs $(\mathrm{k}=4)$ but remaining subgroups were affected by substantial heterogeneity. Inconsistency was downgraded one point.

d. Heterogeneity decreased after removing outlier studies and was explained in RCTs $(\mathrm{k}=5)$ but remaining subgroups were affected by substantial heterogeneity. Inconsistency was downgraded one point.

e. Although the optimal information size was met ( $\mathrm{n}>400$ ), the large $\mathrm{CI}$ around effect estimate with upper and lower limits included no effect. Imprecision was downgraded one point.

f. Heterogeneity was largely explained in non-theory based studies $(\mathrm{k}=2)$ but remaining subgroups were affected by moderate to substantial heterogeneity. Inconsistency was downgraded one point.

g. Heterogeneity was explained after removing high/low effect studies and outlier studies.

h. Heterogeneity reduced after removing high/low effect studies and outlier studies but remained low to moderate. Inconsistency was downgraded one point.

i. Substantial heterogeneity was not explained in the subgroup analysis. Inconsistency was downgraded two points.

j. Heterogeneity was explained after removing high/low effect studies and in CTs.

k. Heterogeneity reduced after removing high/low effect studies but remained moderate. Inconsistency was downgraded one point.

1. Heterogeneity was explained in children $(\mathrm{k}=2)$ but remaining subgroups were affected by substantial heterogeneity. Inconsistency was downgraded one point.

$\mathrm{m}$. Heterogeneity decreased after removing outlier studies but remained moderate. Heterogeneity was downgraded one point. 
intervention frequency may further help explain heterogeneity and should be considered in future studies when pooled effect estimates are based on a larger number of studies (Braithwaite et al., 2011).

\subsection{Main findings-secondary outcomes}

About 38\% of the included studies reported significant results of school-based interventions on PA in favor of the IG. We did not calculate pooled effect estimates due to diversity in PA outcomes, measurement instruments and measurement time across the studies. However, the narrative summary of intervention effects on PA showed that studies reporting increased PA in PE classes predominantly implemented selfdesigned teaching approaches with BCTs in the clusters 'Antecedents', 'Repetition and substitution', 'Comparison of behavior', and 'Shaping knowledge'. In contrast, studies reporting significant results in PA behavior outside of PE and in leisure time most frequently implemented BCTs within the cluster "Natural consequences" followed by "Feedback and monitoring', Repetition and substitution', 'Goals and planning', 'Shaping knowledge', and 'Comparisons of behavior'. This could lead to the assumption that in order to increase situational PA (e.g. in PE) interventions should consider restructuring the physical/social environment or adding objects. When aiming to increase PA behavior over time, interventions should include information about health, social and environmental, or emotional consequences. However, this assumption is based on an uneven distribution of studies using different measurement tools. Future research should further examine which BCTs effectively increase PA of children and adolescents over time.

\subsection{Methodological quality and certainty of evidence}

Every study included in this review had a high risk of bias in more than one domain and was therefore considered at high risk for bias (see additional file 2). The RCT by Salmon et al. (2008) had the highest methodological quality: low risk of bias was found in each category except for blinding of participants/personnel and blinding of outcome assessment. Since school or research staff mainly provided the intervention components, and the primary outcome data were collected using self-report measures, it is not surprising that these categories were rated with a high risk of bias across all reviewed studies. These limitations lie in the nature of such field-based intervention studies and therefore should not be interpreted too strongly. However, the exclusion of these domains would only reduce the number of studies considered at high risk for bias by a factor of four. The remaining 53 studies had at least one other domain that was rated with high risk of bias. For example, random sequence generation and allocation concealment were insufficiently described or followed in most studies, thereby increasing the risk of an unintended intervention in the CG. Furthermore, many studies did not sufficiently report or examine baseline group differences. Of the 57 studies, only 18 studies reported insignificant baseline group differences, which could lead to an overestimation of intervention effects. In order to improve their methodological quality, future studies should make more effort to thoroughly describe randomization and allocation procedures, examine and report potential baseline group differences and attrition rates throughout the trial.

Although not evaluated using the Cochrane risk of bias tool, the diversity of questionnaires used to measure the same motivational outcomes could further affect the summary of results. In particular, several studies examined self-determined or autonomous motivation by calculating the SDI and RAI. Although we assume that both of these indices represent autonomous motivation and behavior is self-regulated, the studies did not calculate the indices uniformly. Additionally, intervention fidelity is not considered in this evaluation tool, which can have an impact on the effectiveness of interventions (Quested et al., 2017). However, on a positive note, several studies providing a teacher training verified intervention fidelity, e.g. by means of a pilot phase (Jones et al., 2010; Moreno-Murcia and Sànchez-Latorre, 2016; Perlman, 2010, 2011,
2015), videotaping or direct observation of lessons (Digelidis et al., 2003; Franco and Coterón, 2017; González-Cutre et al., 2018; Lonsdale et al., 2019; Meng and Keng, 2016; Smith et al., 2015, 2018; Todorovich \& Curtner-Smith, 2002, 2003; Wallhead, Garn, \& Vidoni, 2014; Wallhead and Ntoumanis, 2004; Yli-Piipari et al., 2018), weekly reports by the teachers (Hortigüela Alcalá and Garijo, 2017) or weekly meetings with the researchers (Blatsis et al., 2016; Méndez-Giménez et al., 2015). Additionally, the majority of reviewed studies provided little or no information on the tasks of the CG. It is therefore unclear how exactly the contents of a regular and a modified PE class differed. Future intervention developers are advised to disclose the strategies used to actively change their outcomes of interest in the IG and provide a detailed description of the control condition.

The methodological limitations identified on study level affected the certainty of evidence on outcome level. We applied the GRADE approach (Guyatt et al., 2008; Schünemann et al., 2013) to examine the certainty of evidence on meta-analyzed outcomes. A moderate certainty of evidence was found for ego goal orientation and ego/performance teaching climate; limited certainty of evidence was found for enjoyment, basic psychological needs and the various types of motivation and regulation characterized along the autonomy control continuum (Deci and Ryan, 2000; Ryan and Deci, 2017) including indices such as the SDI and RAI. The reasons for this lie in the downgrading of risk of bias, inconsistency, imprecision, and publication bias. Hence, it cannot be confirmed that the results of the meta-analyses provide reliable evidence on the effectiveness or lack of effectiveness of PA interventions on the majority of the examined motivational outcomes. This must be given special consideration when interpreting the results for the different types of motivation. Significant effects were found for intrinsic motivation, identified regulation and SDI, whereas non-significant effects were found for RAI. These conflicting results should not be interpreted too strongly because confidence in both the non-significant and significant findings is very low to low. In summary, it is not possible to draw an overall conclusion about the effectiveness of PA interventions on the different types of motivation and regulation ranging from amotivation to intrinsic motivation.

In addition to the methodological limitations of the primary studies, limitations at the review level must be addressed. It was not possible to include every study in the meta-analysis of motivational outcomes because several studies did not provide sufficient data to calculate Hedges' $g$. Additionally, we did not perform meta-analysis on PA outcomes and the summary of findings is based on a narrative description. Although the search strategy included many psychological variables linked to motivation, it cannot be assumed that a complete assessment of motivation and associated factors has been made. For example, the term intention was not included in the search strategy despite its relation to PA behavior of children and adolescents (Sallis et al., 2000).The search also did not include non-English articles and grey literature, which may result in an incomplete coverage of all studies in this area. Lastly, we did not perform an in-depth analysis of theory use of the included studies. Although the majority of reviewed studies were described as theory-based, we do not know in which way the interventions were guided by the underlying theory. Therefore, the comparison of the effectiveness of non-theory based studies and theory-based studies in the subgroup analyses must be interpreted with caution. Nevertheless, this systematic review and meta-analysis give first insights into the effectiveness of PA interventions on students' motivation towards PA. Using the previously published and comprehensive search strategy, we were able to review 57 studies using an internationally accepted tool for quality assessment within studies (Higgins and Green, 2011) and evaluated the evidence on outcome level using the GRADE methodology (Schünemann et al., 2013). The large inconsistency of findings give reason for future studies to explore moderating factors of motivation in more detail. Different types of motivation and motivational strategies may be of relevance depending on sex/gender, socio-economic status and exercise habits. 


\section{Conclusion}

The results of this systematic review and meta-analysis suggest that school-based PA interventions may be effective on a variety of motivational outcomes such as enjoyment, perceived autonomy, intrinsic motivation, motivational climate, and goal orientation. The certainty of evidence, however, ranged from very low to moderate, depending on the examined outcome. The narrative synthesis of findings on PA also indicated that school-based PA interventions may increase activity levels of children and adolescents in PE as well as in leisure time.

In order to synthesize information on effective intervention components that were used in the reviewed studies, behavior change techniques were coded using a standardized taxonomy. The analysis revealed that the techniques used to increase motivation towards PA varied across the studies and in relation to the examined outcomes. This was also evident for PA outcomes, where intervention strategies varied depending on the domain where information on PA behavior was collected (in class or in leisure time). Further research is needed to investigate which strategies are truly effective and needed to increase motivation towards PA and PA behavior in children and adolescents. To this end, future intervention developers are advised to describe their intervention components in a standardized manner.

\section{Ethics approval and consent to participate}

Not applicable.

\section{Consent for publication}

Not applicable.

\section{Availability of data and material}

Not applicable.

\section{Declaration of competing interest}

The authors declare that they have no known competing financial interests or personal relationships that could have appeared to influence the work reported in this paper.

\section{Acknowledgements}

The authors thank Denise Renninger for her support in the process of study selection, data extraction, and risk of bias assessment. Furthermore, the authors thank the ESA-Consortium for their support.

\section{Appendix A. Supplementary data}

Supplementary data to this article can be found online at https://doi. org/10.1016/j.psychsport.2020.101770.

\section{Funding}

The study was funded by the Education, Audiovisual and Culture Executive Agency (EACEA) Erasmus + Sport Programme $(E+$ Sport Project - 579661-EPP-1-2016-2-IT-SPO-SCP "Enriched Sport Activities Program" Agreement number 2016-3723/001-001), which had no influence on study design, conduct, analyses, or interpretation of the data, or decision to submit results.

\section{References}

Almolda-Tomas, F., Sevil-Serrano, J., Julian-Clemente, J., Abarca-Sos, A., AibarSolana, A., \& Garcia-Gonzalez, L. (2014). Application of teaching strategies for improving students' situational motivation in physical education. Rev Electron Investig Psicoeduc Psigopedag, 12(2), 391-417.
Ames, C. (1992). Classrooms: goals, structures, and student motivation. Journal of Educational Psychology, 84, 261-271.

Ames, C., \& Archer, J. (1988). Achievement goals in the classroom: students' learning strategies and motivation processes. Journal of Educational Psychology, 80(3), 260-267.

Barzouka, K., Sotiropoulos, K., \& Kioumourtzoglou, E. (2015). The effect of feedback through an expert model observation on performance and learning the pass skill in volleyball and motivation. Journal of Physical Education \& Sport, 15(3), 407-416.

Blatsis, P., Saraslanidis, P., Barkoukis, V., Manou, V., Tzavidas, K., Palla, S., et al. (2016). The effect of IAAF Kids Athletics on the physical fitness and motivation of elementary school students in track and field. Journal of Physical Education and Sport, 16(3), 883-896.

Braga, R. K., De Lima, C. A. F., Moyses, S. J., Moyses, S. T., \& Werneck, R. I. (2017). Virtual games assets: strategy potential to promote health and combat obesity school. Motricidade, 13(S1), 121-128.

Braithwaite, R., Spray, C. M., \& Warburton, V. E. (2011). Motivational climate interventions in physical education: a meta-analysis. Psychology of Sport and Exercise, 12(6), 628-638.

Brankovic, E., \& Hadzikadunic, M. (2017). Physical education experimental program to test the effect on perceived competence. Sport Mont, 15(2), 25-30.

Bunker, D., \& Thorpe, R. D. (1982). A model for the teaching of games in secondary schools. Bulletin of Physical Education, 18, 7-10.

Burns, R. D., Fu, Y., \& Podlog, L. W. (2017). School-based physical activity interventions and physical activity enjyoment: a meta-analysis. Preventive Medicine, 103, 84-90.

* Bush, L. P., Laberge, S., \& Laforest, S. (2010). Physical activity promotion among underserved adolescents: "make it fun, easy, and popular". Health Promot Practice, 11 (3 Suppl. 1), 79S-87S.

Di Cagno, A., Crova, C., \& Pesce, C. (2006). Effects of educational rhythm-based learning on coordinative motor performance and sports enjoyment of male and female pupils. Journal of Human Movement Studies, 51(3), 143-165.

* Chang, Y. K., Chen, S., Tu, K. W., et al. (2016). Effect of autonomy support on selfdetermined motivation in elementary physical education. Journal of Sports Science and Medicine, 15(3), 460-466.

* Chatzisarantis, N. L. D., \& Hagger, M. S. (2009). Effects of an intervention based on selfdetermination theory on self-reported leisure-time physical activity participation. Psychol Health, 24(1), 29-48.

* Chiva-Bartoll, Ó., Salvador-García, C., \& Ruiz-Montero, P. J. (2018). Teaching games for understanding and cooperative learning: can their hybridization increase motivational climate among physical education students?. Croatian Journal of Education, 20(2), 561-584.

* Christodoulidis, T., Papaioannou, A., \& Digelidis, N. (2001). Motivational climate and attitudes towards exercise in Greek senior high school: a year-long intervention. European Journal of Sport Science, 1(4), 1.

Cohen, J. (1988). Statistical Power Analysis for the Behavioral Sciences (second ed.) Hillsdale, NJ: Erlbaum.

* Cuevas, R., García- López, L. M., \& Serra- Olivares, J. (2016). Sport education model and self-determination theory: an intervention in secondary school children. Kinesiology, 48(1), 30-38.

Deci, E. L., \& Ryan, R. M. (2000). The "what" and "why" of goal pursuits: human needs and the self-determination of behavior. Psychological Inquiry, 11(4), 227-268.

Demetriou, Y., \& Honer, O. (2012). Physical activity interventions in the school setting: a systematic review. Psychology of Sport and Exercise, 13(2), 186-196.

Demetriou, Y., Sudeck, G., \& Höner, O. (2014). Indirekte Gesundheitseffekte des Unterrichtsprogramms HealthyPEP. Sportwiss, 44(2), 86-98.

Demetriou, Y., Sudeck, G., Thiel, A., \& Höner, O. (2015). The effects of school-based physical activity interventions on students' health-related fitness knowledge: a systematic review. Educational Research and Reviews, 16, 19-40.

Demetriou, Y., Reimers, A. K., Alesi, M., Scifo, L., Borrego, C., Monteiro, D., et al. (2019). Effects of school-based interventions on motivation towards physical activity in children and adolescents: protocol for a systematic review. Systematic reviews, 8 (113).

* Digelidis, N., Papaioannou, A., Laparidis, K., \& Christodoulidis, T. (2003). A one-year intervention in 7th grade physical education classes aiming to change motivational climate and attitudes towards exercise. Psychology of Sport and Exercise, 4(3), 195-210.

* Dudley, D. A., Okely, A. D., Pearson, P., \& Peat, J. (2010). Engaging adolescent girls from linguistically diverse and low income backgrounds in school sport: a pilot randomised controlled trial. Journal of Science and Medicine in Sport, 13(2), 217-224.

Dudley, D., Okely, A., Pearson, P., \& Cotton, W. (2011). A systematic review of the effectiveness of physical education and school sport interventions targeting physical activity, movement skills and enjoyment of physical activity. European Physical Education Review, 17(3), 353-378.

Eime, R. M., Young, J. A., Harvey, J. T., Charity, M. J., \& Payne, W. R. (2013), A systematic review of the psychological and social benefits of participation in sport for children and adolescents: informing development of a conceptual model of health through sport. International Journal of Behavioral Nutrition and Physical Activity, 10 (98), 98.

* Elbe, A. M., Wikman, J. M., Zheng, M., Larsen, M. N., Nielsen, G., \& Krustrup, P. (2017). The importance of cohesion and enjoyment for the fitness improvement of 8-10-yearold children participating in a team and individual sport school-based physical activity intervention. European Journal of Sport Science, 17(3), 343-350.

Epstein, J. L. (1989). Family structures and student motivation: a developmental perspective. In C. Ames, \& R. Ames (Eds.), Research on Motivation in Education (vol. 3, pp. 259-295). New York: Academic Press.

Fairclough, S., \& Stratton, G. (2005). Improving health-enhancing physical activity in girls' physical education. Health Education Research, 20(4), 448-457. 
* Fairclough, S. J., McGrane, B., Sauders, G., Taylor, S., Owen, M., \& Curry, W. (2016). A non-equivalent group pilot trial of a school-based physical activity and fitness intervention for 10-11 year Old English children: born to move. BMC Public Health, 16(1).

* Franco, E., \& Coterón, J. (2017). The effects of a physical education intervention to support the satisfaction of basic psychological needs on the motivation and intentions to Be physically active. Journal of Human Kinetics, 59(1), 5-15.

* Fu, Y., Gao, Z., Hannon, J. C., Schultz, B., Newton, M., \& Sibthorp, J. (2013). Influence of a health-related physical fitness model on students' physical activity, perceived competence, and enjoyment. Perceptual \& Motor Skills, 117(3), 956-970.

* Fu, Y., Gao, Z., Hannon, J. C., Burns, R. D., \& Brusseau, T. A. (2016). Effect of the SPARK program on physical activity, cardiorespiratory endurance, and motivation in middle-school students. Journal of Physical Activity and Health, 13(5), 534-542.

* González-Cutre, D., Sierra, A. C., Beltrán-Carrillo, V. J., Peláez-Pérez, M., \& Cervelló, E. (2018). A school-based motivational intervention to promote physical activity from a self-determination theory perspective. Journal of Education Research, 111(3), 320-330.

* Gorely, T., Nevill, M. E., Morris, J. G., Stensel, D. J., \& Nevill, A. (2009). Effect of a school-based intervention to promote healthy lifestyles in 7-11 year old children. International Journal of Behavioral Nutrition and Physical Activity, 6, 5.

Goudas, M., \& Biddle, S. (1994). Perceived motivational climate and intrinsic motivation in school physical education classes. European Journal of Psychology of Education, 9 (3), 241-250.

Guyatt, G. H., Oxman, A. D., Vist, G. E., Kunz, R., Falck-Ytter, Y., Alonso-Coello, P., et al. (2008). GRADE: an emerging consensus on rating quality of evidence and strength of recommendations. BMJ, 336(7650), 924-926.

Hallal, P. C., Andersen, L. B., Bull, F. C., Guthold, R., Haskell, W., Ekelund, U., et al. (2012). Global physical activity levels: surveillance progress, pitfalls, and prospects. Lancet, 380(9838), 247-257.

* Harrington, D. M., Davies, M. J., Bodicoat, D. H., Charles, J. M., Chudasama, Y. V., Gorely, T., et al. (2018). Effectiveness of the 'Girls Active' school-based physical activity programme: a cluster randomised controlled trial. International Journal of Behavioral Nutrition and Physical Activity, 15(1), 40.

Van Hecke, L., Loyen, A., Verloigne, M., van der Ploeg, H. P., Lakerveld, J., Brug, J., et al (2016). Variation in population levels of physical activity in European children and adolescents according to cross-European studies: a systematic literature review within DEDIPAC. International Journal of Behavioral Nutrition and Physical Activity, 13,70 .

Hedges, L. V., \& Olkin, I. (1985). Statistical Methods for Meta-Analysis. Sand Diego, CA: Academic Press.

Hedges, L. V., \& Vevea, J. L. (1998). Fixed and Random Effects Models in Meta-Analysis. New York: Russel Sage Foundation.

Higgins, J. P. T., \& Green, S. (Eds.). (2011). Cochrane Handbook for Systematic Reviews of Interventions. The Cochrane Collaboration [updated March 2011] Version 5.1.0. .

Higgins, J. P., Thompson, S. G., Deeks, J. J., \& Altman, D. G. (2003). Measuring inconsistency in meta-analyses. BMJ, 327(7414), 557-560.

Higgins, J. P. T., Altman, D. G., Gøtzsche, P. C., Jüni, P., Moher, D., Oxman, A. D., et al. (2011). The Cochrane Collaboration's tool for assessing risk of bias in randomised trials. BMJ, 343.

* Hobin, T. M., Subramaniam, P. R., \& Wuest, D. A. (2018). The impact of a novel sports activity on student motivation in high school physical education. International Journal of Physical Education, 55(1), 15-27.

* Hortigüela Alcalá, D., \& Garijo, A. H. (2017). Teaching games for understanding: a comprehensive approach to promote student's motivation in physical education. Journal of Human Kinetics, 59(1), 17-27.

Ickes, M. J., Erwin, H., \& Beighle, A. (2013). Systematic review of recess interventions to increase physical activity. Journal of Physical Activity and Health, 10(6), 910-926.

* Jamner, M., Spruijt-Metz, D., Bassin, S., \& Cooper, D. (2004). A controlled evaluation of a school-based intervention to promote physical activity among sedentary adolescent females: Project FAB. Journal of Adolescent Health, 34(4), 279-289.

Janssen, I., \& Leblanc, A. G. (2010). Systematic review of the health benefits of physical activity and fitness in school-aged children and youth. International Journal of Behavioral Nutrition and Physical Activity, 7(1), 40.

* Jones, R., Marshall, S., \& Peters, D. (2010). Can we play a game now? The intrinsic benefits of TGfU. European Journal of Physical and Health Education, 4(2), 57-63.

* Kokkonen, J., Yli-Piipari, S., Kokkonen, M., \& Quay, J. (2018). Effectiveness of a creative physical education intervention on elementary school students' leisure-time physical activity motivation and overall physical activity in Finland. European Physical Education Review, 25(3).

* Lonsdale, C., Lester, A., Owen, K. B., White, R. L., Peralta, L., Kirwan, M., et al. (2019). An internet-supported school physical activity intervention in low socioeconomic status communities: results from the Activity and Motivation in Physical Education (AMPED) cluster randomised controlled trial. British Journal of Sports Medicine, 53 (6), 341-347. https://doi.org/10.1136/bjsports-2017-097904.

* Lonsdale, C., Rosenkranz, R. R., Sanders, T., Peralta, L., Bennie, A., Jackson, B., et al (2013). A cluster randomized controlled trial of strategies to increase adolescents' physical activity and motivation in physical education: results of the Motivating Active Learning in Physical Education (MALP) trial. Preventive Medicine, 57(5), 696-702.

* Méndez-Giménez, A., Fernández-Río, J., \& Méndez-Alonso, D. (2015). Sport education model versus traditional model: effects on motivation and sportsmanship. Revista Internacional de Medicina y Ciencias de la Actividad Fisica y del Deporte, 15(59), 449-466.

* Meng, H. Y., \& Keng, J. W. C. (2016). The effectiveness of an autonomy-supportive teaching structure in physical education. RICYDE. Revista internacional de ciencias del deporte, 12(43), 5-28.
* Meng, H. Y., Whipp, P., Dimmock, J., \& Jackson, B. (2013). The effects of choice on autonomous motivation, perceived autonomy support, and physical activity levels in high school physical education. Journal of Teaching in Physical Education, 32(2), 131-148.

Michie, S., Richardson, M., Johnston, M., Abraham, C., Francis, J., Hardeman, W., et al. (2013). The behavior change technique taxonomy (v1) of 93 hierarchically clustered techniques: building an international consensus for the reporting of behavior change interventions. Annals of Behavioral Medicine, 46(1), 81-95.

* Miller, A., Christensen, E., Eather, N., Gray, S., Sproule, J., Keay, J., et al. (2016). Can physical education and physical activity outcomes be developed simultaneously using a game-centered approach?. European Physical Education Review, 22(1), $113-133$.

* Moreno, J. A., Gonzalez-Cutre, D., Martin-Albo, J., \& Cervello, E. (2010). Motivation and performance in physical education: an experimental test. Journal of Sports Science and Medicine, 9(1), 79-85.

* Moreno-Murcia, J. A., \& Sànchez-Latorre, F. (2016). The effects of autonomy support in physical education classes. RICYDE. Revista internacional de ciencias del deporte, 12 (43), 79-89.

* Nation-Grainger, S. (2017). "It's just PE 'till' it felt like a computer game": using technology to improve motivation in physical education. Research Papers in Education, 32(4), 463-480.

Ng, J. Y. Y., Ntoumanis, N., Thøgersen-Ntoumani, C., Deci, E. L., Ryan, R. M., Duda, J. L., et al. (2012). Self-determination theory applied to health contexts. Perspectives on Psychological Science, 7(4), 325-340.

Owen, K., Smith, J., Lubans, D. R., Ng, J. Y. Y., \& Lonsdale, C. (2014). Self-determined motivation and physical activity in children and adolescents: a systematic review and meta-analysis. Preventive Medicine, 67, 270-279.

* Palmer, S. E., Bycura, D. K., \& Warren, M. (2018). A physical education intervention effects on correlates of physical activity and motivation. Health Promot Practice, 19 (3), 455-464.

* Pardo, B. M., Bengoechea, E. G., Clemente, J. A. J., \& Lanaspa, E. G. (2016). Motivational outcomes and predictors of moderate-to-vigorous physical activity and sedentary time for adolescents in the sigue La huella intervention. International Journal of Behavioral Medicine, 23(2), 135-142.

Pate, R. R., \& O'Neill, J. R. (2009). After-school interventions to increase physical activity among youth. British Journal of Sports Medicine, 43(1), 14-18.

* Patmanoglou, S., Mantis, K., Digelidis, N., et al. (2008). The command and self-check styles for more effective teaching of tennis at the elementary school. International Journal of Physical Education, 45(1), 26-32.

Penedo, F. J., \& Dahn, J. R. (2005). Exercise and well-being: a review of mental and physical health benefits associated with physical activity. Current Opinion in Psychiatry, 18(2), 189-193.

* Perlman, D. (2010). Change in affect and needs satisfaction for amotivated students within the Sport Education Model. Journal of Teaching in Physical Education, 29(4), 433-445.

* Perlman, D. J. (2011). Examination of self-determination within the sport education model. Asia-Pacific Journal of Health Sport \& Physical Education, 2(1), 79-92.

* Perlman, D. J. (2015). Help motivate the amotivated by being a supportive teacher. Physical Education \& Sport Pedagogy, 20(2), 204-214.

* Prusak, K. A., Treasure, D. C., Darst, P. W., \& Pangrazi, R. P. (2004). The effects of choice on the motivation of adolescent girls in physical education. Journal of Teaching in Physical Education, 23(1), 19-29.

Quested, E., Ntoumanis, N., Thogersen-Ntoumani, C., Hagger, M. S., \& Hancox, J. E. (2017). Evaluating quality of implementation in physical activity interventions based on theories of motivation: current challenges and future directions. International Review of Sport and Exercise Psychology, 10(1), 252-269.

Ryan, R. M., \& Deci, E. L. (2017). Self-Determination Theory: Basic Pyschological Needs in Motivation, Development, and Wellness (p. 756). New York; London: The Guilford Press.

Ryan, R. M., Patrick, H., Deci, E. L., \& Williams, G. C. (2008). Facilitating health behavior change and its maintenance: interventions based on Self-Determination Theory. The European Health Psychologist, 10(1), 2-5.

Sallis, J. F., Prochaska, J. J., \& Taylor, W. C. (2000). A review of correlates of physical activity of children and adolescents. Medicine \& Science in Sports \& Exercise, 32(5), 963-975.

* Salmon, J., Hume, M., \& Crawford, D. (2008). Outcomes of a group-randomized trial to prevent excess weight gain, reduce screen behaviours and promote physical activity in 10-year-old children: switch-play. International Journal of Obesity, 32(4), 601-612.

Schünemann, H. B. J., Guyatt, G., \& Oxman, A. (Eds.). (2013). GRADE Handbook for Grading the Quality of Evidence and the Strength of Recommendations. GRADE Working Group (updated October 2013).

* Sevil, J., Abos, A., Aibar, A., Julián, J. A., \& Garcia-Gonzalez, L. (2016). Gender and corporal expression activity in physical education: effect of an intervention on students' motivational processes. European Physical Education Review, 22(3), 372-389.

* Shannon, S., Brennan, D., Hanna, D., Younger, Z., Hassan, J., \& Breslin, G. (2018). The effect of a school-based intervention on physical activity and well-being: a nonrandomised controlled trial with children of low socio-economic status. Sports Med Open, 4(1), 16

Siedentop, D. (1994). Sport Education : Quality PE through Positive Sport Experiences. Champaign, IL: Human Kinetics Publishers.

Singh, A., Uijtdewilligen, L., Twisk, J. W., van Mechelen, W., \& Chinapaw, M. J. (2012). Physical activity and performance at school-A systematic review of the literature including a methodological quality assessment. Archives of Pediatrics and Medicine, 166(1), 49-55. 
van Sluijs, E. M., McMinn, A. M., \& Griffin, S. J. (2007). Effectiveness of interventions to promote physical activity in children and adolescents: systematic review of controlled trials. BMJ, 335(7622), 703.

* Smith, L., Harvey, S., Savory, L., Fairclough, S., Kozub, S., \& Kerr, C. (2015). Physica activity levels and motivational responses of boys and girls: a comparison of direct instruction and tactical games models of games teaching in physical education. European Physical Education Review, 21(1), 93-113.

* Smith, J. J., Beauchamp, M. R., Faulkner, G., Morgan, P. J., Kennedy, S. G., \& Lubans, D. R. (2018). Intervention effects and mediators of well-being in a schoolbased physical activity program for adolescents: the 'Resistance Training for Teens' cluster RCT. Mental Health and Physical Activity, 15, 88-94.

* Spittle, M., \& Byrne, K. (2009). The influence of sport education on student motivation in physical education. Physical Education and Sport Pedagogy, 14(3), 253-266.

van Stralen, M. M., Yildirim, M., te Velde, S. J., Brug, J., van Mechelen, W., Chinapaw, M. J., et al. (2011). What works in school-based energy balance behaviour interventions and what does not? A systematic review of mediating mechanisms. International Journal of Obesity, 35(10), 1251-1265.

* Todorovich, J., R., \& Curtner-Smith, M., D. (2002). Influence of the motivational climate in physical education on sixth grade pupils' goal orientations. European Physical Education Review, 8(2), 119-138.

* Todorovich, J. R., \& Curtner-Smith, M. D. (2003). Influence of the motivational climate in physical education on third grade students' task and ego orientations. Journal of Classroom Interaction, 38(1), 36-46.
Vallerand, R. J. (1997). Toward A hierarchical model of intrinsic and extrinsic motivation. Advances in Experimental Social Psychology, 29, 271-360.

* Wallhead, T., L, Garn, A., C., \& Vidoni, C. (2014). Effect of a sport education program on motivation for physical education and leisure-time physical activity. Research Quarterly for Exercise \& Sport, 85(4), 478-487.

* Wallhead, T. L., \& Ntoumanis, N. (2004). Effects of a sport education intervention on students' motivational responses in physical education. Journal of Teaching in Physical Education, 23(1), 4-18.

* Wang, J. J., Baranowski, T., Lau, P. W. C., Buday, R., \& Gao, Y. (2017). Story immersion may Be effective in promoting diet and physical activity in Chinese children. Journal of Nutrition Education and Behavior, 49(4), 321-329.e1.

* Weigand, Daniel, A., \& Burton, Shane (2002). Manipulating achievement motivation in physical education by manipulating the motivational climate. European Journal of Sport Science, 2(1), 1.

World Health Organization. (2010). Global recommendations on physical activity for health. Geneva: WHO Press.

* Yli-Piipari, S., Layne, T., Hinson, J., \& Irwin, C. (2018). Motivational pathways to leisure-time physical activity participation in urban physical education: a clusterrandomized trial. Journal of Teaching in Physical Education, 37(2), 123-132.

* Zach, S., Raviv, T., \& Meckel, Y. (2016). Using information communication technologies (ICTs) for motivating female adolescents to exercise/run in their leisure time. Computers in Human Behavior, 60, 593-601. 